\title{
ANÁLISE DE EXPERIMENTOS EM QUADRADO LATINO COM NÚMERO DIFERENTE DE OBSERVAÇÕES POR UNIDADE EXPERIMENTAL
}

Tese apresentada à Escola Superior de Agricultura "Luiz de Queiroz", da Universidade de São Paulo, para obtenção do título de Doutor em Agronomia. Área de Concentração: Estatística e Experimentação Agronômica.

PIRACICABA

Estado de São Paulo - Brasil

Janeiro - 1996 


\section{Dados Internacionais de Catalogação na Publicação (CIP) \\ DIVISÃo DE BIBLIOTECA E DOCUMENTAÇẤO - Campus "Luiz de Queiroz"/USP}

Piedade, Sônia Maria de Stefano

Análise de experimentos em quadrado latino com número diferente de observações

por unidade experimental / Sônia Maria de Stefano Piedade. - Piracicaba, 1996.

150p. : it.

Tese (doutorado) - Escola Superior de Agricultura Luiz de Queiroz, 1996.

Bibliografia.

1. Análise de variância 2. Delineamento de experimento 3. Experimento agrícola 1. Título

CDD 519.5

519.535 


\section{ANÁLISE DE EXPERIMENTOS EM QUADRÁDO LATINO COM NÚMERO DIFERENTE DE OBSERVAÇÕES POR UNIDADE EXPERIMENTAL}

SÔNIA MARIA DE STEFANO PIEDADE Engenheiro Agrônomo

Orientador : Prof. Dr. Humberto de Campos

Tese apresentada à Escola Superior de Agricultura "Luiz de Queiroz", da Universidade de São Paulo, para obtenção do título de Doutor em Agronomia. Área de Concentração: Estatística e Experimentação Agronômica.

PIRACICABA

Estado de São Paulo - Brasil

Janeiro - 1996 


\title{
ANÁLISE DE EXPERIMENTOS EM QUADRADO LATINO COM NÚMERO DIFERENTE DE OBSERVAÇÕES POR UNIDADE EXPERIMENTAL
}

\author{
SÔNIA MARIA DE STEFANO PIEDADE
}

Aprovado em: 07/03/96

Comissão Julgadora:

Prof. Dr. Humberto de Campos

Prof. Dr. Décio Barbin

Prof. Dr. Antonio Francisco Iemma

Prof. Dr. David Ariovaldo Banzatto

Prof. Dr. Gilnei de Souza Duarte
ESALQ/Piracicaba ESALQ/Piracicaba ESALQ/Piracicaba FCAV/Jaboticabal UFAL/Lavras

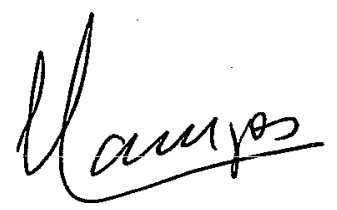

Prof. Dr. Humberto de Campos Orientador 
Aos meus pais,

que fizeram de suas vidas um

exemplo para a minha ,

AGRADEÇO .

Ao Miguel,

pelo apoio e compreensão ,

DEDICO . 


\section{AGRADECIMENTOS}

Ao Professor Dr. Humberto de Campos, pela orientação e, acima de tudo, pela amizade .

Ao $\mathrm{CNPq}$, pela bolsa de estudo concedida .

Ao extinto Programa Nacional de Melhoramento da Cana-de-açúcar PLANALSUCAR, onde adquiri meus conhecimentos práticos de estatística experimental e cultivei muitas amizades .

Ao Professor Dr. Antonio Francisco Iemma, pelas valiosas sugestões e pelo incentivo .

Ao colega Professor Samuel Tanaami, pela acolhida e pelos desabafos .

Aos demais professores do Departamento de Matemática e Estatística da Escola Superior de Agricultura "Luiz de Queiroz" , de modo especial, Prof. Dr. Décio Barbin, Prof ${ }^{t}$. Dr ${ }^{a}$. Maria Cristina Stolf Nogueira, Prof ${ }^{a}$. $\mathrm{Dr}^{\mathrm{a}}$. Clarice Garcia Borges Demétrio.

Ao colega Professor Dr. José Orlando Filho, do Centro de Ciências Agrárias Araras, da Universidade Federal de São Carlos, pelos dados experimentais utilizados neste trabalho .

Aos funcionários do Departamento de Matemática e Estatística da Escola Superior de Agricultura "Luiz de Queiroz", pela paciência . Aos colegas do Curso de Pós-graduação, pela convivência .

A todos que, direta ou indiretamente, colaboraram para a realização deste trabalho . 


\section{ÍNDICE}

Pág.

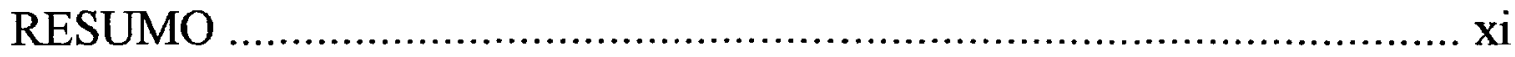

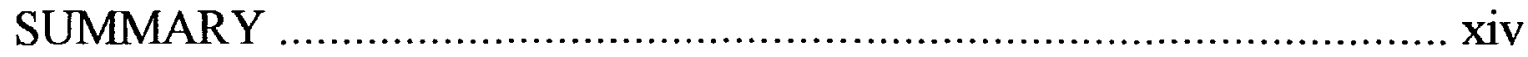

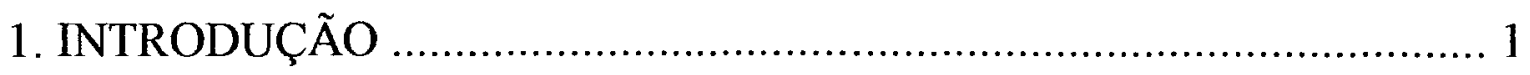

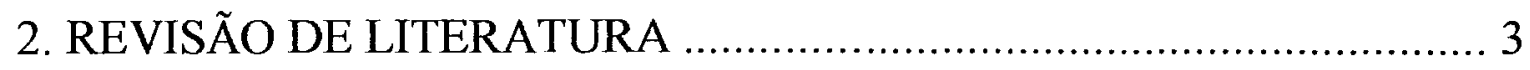

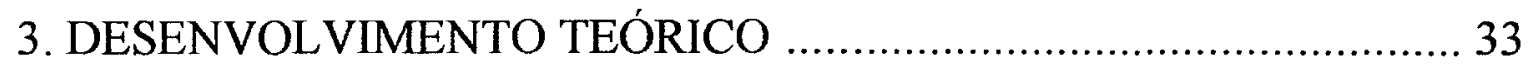

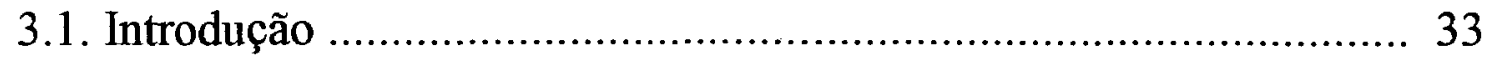

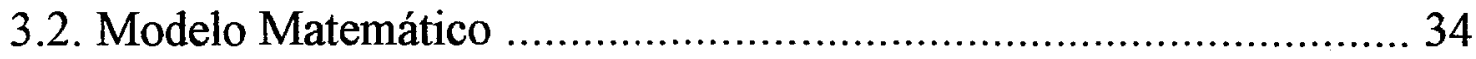

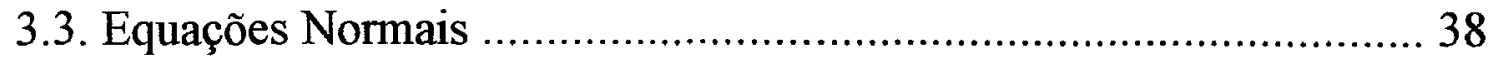

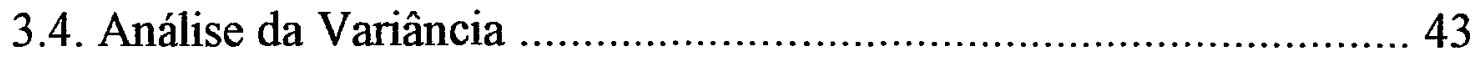

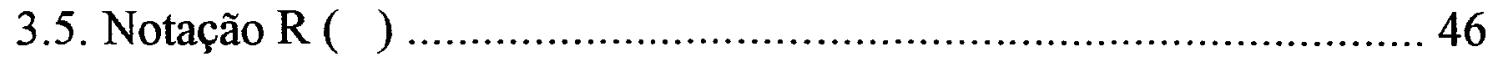

3.5.1. Redução na soma de quadrados total devida ao ajuste da constante $\mu$.................................. 48

3.5.2. Redução na soma de quadrados total devida ao ajuste de linhas após o ajuste da constante 48

3.5.3. Redução na soma de quadrados total devida ao ajuste de colunas após o ajuste da constante 49

3.5.4. Redução na soma de quadrados total devida ao ajuste de tratamentos após o ajuste da constante 
vii.

3.5.5. Redução na soma de quadrados total devida ao ajuste de linhas após o ajuste de colunas e da constante

3.5.6. Redução na soma de quadrados total devida ao ajuste de linhas após o ajuste de tratamentos e da constante

3.5.7. Redução na soma de quadrados total devida ao ajuste de colunas após o ajuste de linhas e da constante 53

3.5.8. Redução na soma de quadrados total devida ao ajuste de colunas após o ajuste de tratamentos e da constante 54

3.5.9. Redução na soma de quadrados total devida ao ajuste de tratamentos após o ajuste de linhas e da constante 55

3.5.10. Redução na soma de quadrados total devida ao ajuste de tratamentos após o ajuste de colunas e da constante 
viii.

3.5.11. Redução na soma de quadrados total devida ao ajuste de linhas após o ajuste de colunas, de tratamentos e da constante

3.5.12. Redução na soma de quadrados total devida ao ajuste de colunas após o ajuste de linhas, de tratamentos e da constante 56

3.5.13. Redução na soma de quadrados total devida ao ajuste de tratamentos após o ajuste de linhas, de colunas e da constante 56

3.6. Tipos de Funções Estimáveis ................................................... 56

3.6.1. Forma geral de funções estimáveis .................................. 57

3.6.2. Funções estimáveis do tipo I ......................................... 59

3.6.3. Funções estimáveis do tipo II ............................................ 64

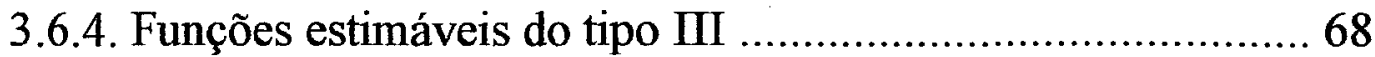

3.6.5. Funções estimáveis do tipo IV ........................................ 72

3.7. Hipóteses Testadas ............................................................... 77

3.7.1. Ordenação : Linha, Coluna , Tratamento ........................ 77

3.7.2. Ordenação : Linha, Tratamento, Coluna ........................... 79

3.7.3. Ordenação : Coluna, Tratamento , Linha ........................... 80

3.7.4. Ordenação : Tratamento , Linha , Coluna ........................ 81 
ix.

3.8. Tipos de Somas de Quadrados ............................................ 81

3.8.1. Somas de quadrados do tipo I ................................... 82

a) Ordenação : Linha , Coluna , Tratamento .................... 82

b) Ordenação : Linha , Tratamento , Coluna ...................... 83

c) Ordenação : Coluna , Tratamento , Linha ..................... 83

d) Ordenação : Tratamento , Linha , Coluna ...................... 84

3.8.2. Somas de quadrados do tipo II ...................................... 84

a) Ordenação : Linha , Coluna , Tratamento .................... 85

b) Ordenação : Linha , Tratamento, Coluna ..................... 85

c) Ordenação : Coluna , Tratamento , Linha ..................... 86

d) Ordenação : Tratamento , Linha , Coluna ..................... 86

3.8.3. Somas de quadrados do tipo III ..................................... 87

3.8.4. Somas de quadrados do tipo IV ................................... 87

4. EXEMPLO ILUSTRATIVO _................................................. 88

4.1. Análises da Variância Segundo os Tipos de

Somas de Quadrados e Ordenações dos

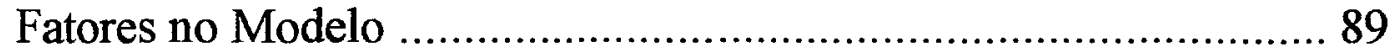

4.1.1. Ordenação : Linha , Coluna , Tratamento ........................ 90

4.1.2. Ordenação : Linha , Tratamento , Coluna .......................... 91

4.1.3. Ordenação : Coluna , Tratamento , Linha ....................... 92

4.1.4. Ordenação : Tratamento , Linha , Coluna ......................... 93

4.2. Funções Estimáveis para Tratamentos ..................................... 94

4.2.1. Ordenação : Linha , Coluna , Tratamento ........................ 94 
4.2.2. Ordenação : Linha, Tratamento, Coluna ........................ 95

4.2.3. Ordenação : Coluna , Tratamento , Linha ........................ 96

4.2.4. Ordenação : Tratamento , Linha , Coluna ......................... 97

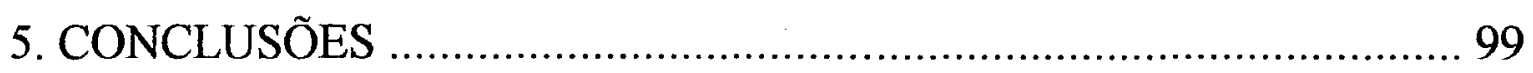

6. REFERÊNCIAS BIBLIOGRÁFICAS .................................... 101

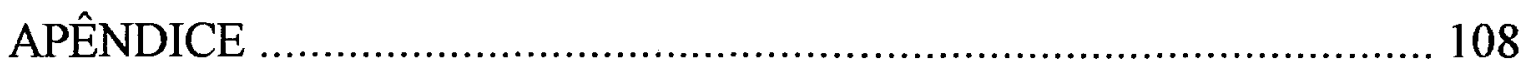


xi.

\section{ANÁLISE DE EXPERIMENTOS EM QUADRADO LATINO \\ COM NÚMERO DIFERENTE DE OBSERVAÇÕES POR \\ UNIDADE EXPERIMENTAL}

Autor : Sônia Maria De Stefano Piedade

Orientador : Prof. Dr. Humberto de Campos

RESUMO

O presente estudo teve por objetivo estruturar as análises

da variância para os experimentos em quadrados latinos com número diferente de observações por unidade experimental.

Para a análise consideraram-se, como em SCHEFFÉ (1959), os seguintes modelos lineares :

$$
\begin{aligned}
& \mathrm{y}_{\mathrm{ijkr}}=\mu+\alpha_{\mathrm{i}}+\beta_{\mathrm{j}}+\mathrm{t}_{\mathrm{k}}+\mathrm{e}_{\mathrm{ijkr}} ; \\
& \mathrm{y}_{\mathrm{ijkr}}=\mu+\alpha_{\mathrm{i}}+\mathrm{t}_{\mathrm{k}}+\beta_{\mathrm{j}}+\mathrm{e}_{\mathrm{ijkr}} ; \\
& \mathrm{y}_{\mathrm{ijkr}}=\mu+\beta_{\mathrm{j}}+\mathrm{t}_{\mathrm{k}}+\alpha_{\mathrm{i}}+\mathrm{e}_{\mathrm{ijkr}} ;
\end{aligned}
$$


xii.

$$
\begin{gathered}
\mathrm{y}_{\mathrm{ijkr}}=\mu+\mathrm{t}_{\mathrm{k}}+\alpha_{\mathrm{i}}+\beta_{\mathrm{j}}+\mathrm{e}_{\mathrm{ijkr}} ; \\
\left(\mathrm{i}=1,2, \ldots, \mathrm{I} ; \mathrm{j}=1,2, \ldots, \mathrm{J} ; \mathrm{k}=1,2, \ldots \mathrm{K} ; \mathrm{r}=1,2, \ldots, \mathrm{n}_{\mathrm{ijk}}\right) \\
(\mathrm{I}=\mathrm{J}=\mathrm{K})
\end{gathered}
$$

onde:

$\mathrm{y}_{\mathrm{ijkr}}$ é o r-ésimo valor observado na unidade experimental referente à iésima linha, na j-ésima coluna e no k-ésimo tratamento ;

$\mu \quad$ é uma constante inerente a todas as observações ;

$\alpha_{i}$ é o efeito da i-ésima linha ;

$\beta_{\mathrm{j}}$ é o efeito da j-ésima coluna;

$\mathrm{t}_{\mathrm{k}}$ é o efeito do k-ésimo tratamento;

$e_{\mathrm{ijkr}}$ é o erro aleatório atribuído à observação $\mathrm{y}_{\mathrm{ijkr}}$ tal que

$$
e_{i j k r} \cap \operatorname{NID}\left(0, \sigma_{e}^{2}\right) .
$$

Discutiram-se os quatro tipos de somas de quadrados fornecidas pelo sistema estatístico SAS, e conseqüentemente, as respectivas hipóteses testadas através delas.

Nesse contexto pode-se concluir que o delineamento em quadrado latino mostrou-se robusto ao desbalanceamento, pois é possível testar através do modelo $\mathrm{y}_{\mathrm{ijkr}}=\mu+\alpha_{\mathrm{i}}+\beta_{\mathrm{j}}+\mathrm{t}_{\mathrm{k}}+\mathrm{e}_{\mathrm{ijkr}}$, hipóteses do tipo : 
xiii.

$$
\mathrm{H}_{0}: \mathrm{t}_{1}=\mathrm{t}_{2}=\ldots=\mathrm{t}_{\mathrm{K}}
$$

Como o modelo é sem interação e como no caso não há casela vazia, somente as somas de quadrados do tipo I são diferentes das somas de quadrados dos tipos II, III e IV.

Ao pesquisador, de posse destas informações, cabe escolher quais funções estimáveis são adequadas para representar a hipótese de seu interesse.

Apresentou-se um exemplo ilustrativo na área agronômica . 
xiv.

\section{ANALYSIS OF EXPERIMENTS IN LATIN SQUARE DESIGN WITH DIFFERENT NUMBER OF OBSERVATIONS PER \\ EXPERIMENTAL UNIT}

Author : Sônia Maria De Stefano Piedade Adviser : Prof. Dr. Humberto de Campos

\section{SUMMARY}

The aim of the present study was to develop the structures of analysis of variance for latin square designs with different number of observations in each experimental unit.

For the analysis the linear models considered, as in SCHEFFÉ (1959), were:

$$
\begin{aligned}
& \mathrm{y}_{\mathrm{ijkr}}=\mu+\alpha_{\mathrm{i}}+\beta_{\mathrm{j}}+\mathrm{t}_{\mathrm{k}}+\mathrm{e}_{\mathrm{ijkr}} ; \\
& \mathrm{y}_{\mathrm{ijkr}}=\mu+\alpha_{\mathrm{i}}+\mathrm{t}_{\mathrm{k}}+\beta_{\mathrm{j}}+\mathrm{e}_{\mathrm{ijkr}} ; \\
& \mathrm{y}_{\mathrm{ijkr}}=\mu+\beta_{\mathrm{j}}+\mathrm{t}_{\mathrm{k}}+\alpha_{\mathrm{i}}+\mathrm{e}_{\mathrm{ijkr}} ;
\end{aligned}
$$


XV.

$$
\mathrm{y}_{\mathrm{ijkr}}=\mu+\mathrm{t}_{\mathrm{k}}+\alpha_{\mathrm{i}}+\beta_{\mathrm{j}}+\mathrm{c}_{\mathrm{ijkr}}
$$

$$
\begin{gathered}
\left(\mathrm{i}=1,2, \ldots, \mathrm{I} ; \mathrm{j}=1,2, \ldots, \mathrm{J} ; \mathrm{k}=1,2, \ldots \mathrm{K} ; \mathrm{r}=1,2, \ldots, \mathrm{n}_{\mathrm{ijk}}\right) \\
(\mathrm{I}=\mathrm{J}=\mathrm{K})
\end{gathered}
$$

where:

$\mathrm{y}_{\mathrm{ijkr}}$ is the r-th observed value in the $\mathrm{i}$-th row, $\mathrm{j}$-th columm and $\mathrm{k}$-th treatment ;

$\mu$ is the constant inherent to all observations ;

$\alpha_{i}$ is the effect of the $i-t h$ row ;

$\beta_{j}$ is the effect of the $j$-th columm ;

$t_{k}$ is the effect of $k$-th treatment ;

$\mathrm{e}_{\mathrm{ijkr}}$ is the random error inherent to $\mathrm{y}_{\mathrm{ijkr}}$ observation, $e_{i j k r} \cap \operatorname{NID}\left(0, \sigma_{e}^{2}\right)$

Four types of sums of squares and the associated hypothesis tested given by the statistical software SAS were discussed.

It was possible to see that the latin square design is robust to unbalancing because it is possible through the model $y_{\mathrm{ijks}}=\mu+\alpha_{\mathrm{i}}+\beta_{\mathrm{j}}+\mathrm{t}_{\mathrm{k}}+\mathrm{e}_{\mathrm{ijkr}}$ to test hypothesis of the type

$$
\mathrm{H}_{0}: \mathrm{t}_{1}=\mathrm{t}_{2}=\ldots=\mathrm{t}_{\mathrm{K}}
$$


xvi.

As the model is without interaction and there is no empty cell, only the type I sum of squares is different from types II, III and IV.

The choice of which case and therefore which estimable function to use will depend on it interpretability and on the hypothesis of the researcher interest .

An illustrative example in agronomic area was presented. 


\section{INTRODUÇÃO}

Os experimentos em quadrado latino têm sua aplicação mais comum na experimentação animal mas, freqüentemente, são usados também na experimentação agricola, na indústria e em muitas outras áreas, quando se depara com a necessidade de um duplo controle local.

$\mathrm{O}$ quadrado latino não é um delineamento flexível $\mathrm{e}$, portanto, tão usual como aquele em blocos casualizados. Pela sua própria configuração, cada linha e cada coluna tem a estrutura de um bloco. Desta forma, se são I tratamentos, o número total de unidades experimentais é I x I e cada tratamento aparece uma única vez em cada linha e em cada coluna.

Os quadrados latinos mais usados são aqueles envolvendo de 5 a 8 tratamentos pois, à medida em que aumenta o número de tratamentos, o tamanho do experimento aumenta rapidamente tornando-se, em alguns casos, impraticável.

Os quadrados latinos $3 \times 3$ e $4 \times 4$ não são vantajosos, devido ao reduzido número de graus de liberdade do resíduo. Entre as alternativas para resolver este problema estão o aumento do número de observações por casela e a repetição dos quadrados latinos. Algumas vezes ocorre do pesquisador ter duas ou mais observações por unidade experimental, e, não raro, com número diferente de observações por casela. 
A literatura sobre qual o melhor procedimento a ser adotado neste caso, é escassa.

$\mathrm{Na}$ revisão bibliográfica, não se encontrou qualquer trabalho que abordasse especificamente este caso de desbalanceamento. Assim, a proposta deste trabalho é fazer uma abordagem sobre a estrutura da análise do delineamento em quadrado latino, com número diferente de observações por unidade experimental interpretando as hipóteses testadas através do PROC GLM (General Linear Models) do sistema estatístico SAS (Statistical Analysis System), por ser dos sistemas o mais usado em casos de desbalanceamento, bem como apresentar exemplo de aplicação. 


\section{REVISÃO DE LITERATURA}

Os primeiros trabalhos com delineamento em quadrado latino são de Euler (1782) e estão citados em PEARCE (1976). Devido a Euler (1782) se atribui o nome quadrado latino pelo fato do autor usar letras latinas para representar os tratamentos em estudo no delineamento.

Posteriormente, OSTLE (1956), citando Fisher (1934), apresenta o quadrado latino como um delineamento experimental baseado numa classificação tripla - ortogonal de linhas, colunas e tratamentos.

Para Yates (1936), citado por PEARCE (1976), no delineamento em quadrado latino, embora a distribuição dos tratamentos seja aleatória, ela é restrita em decorrência do agrupamento dos mesmos em linhas e colunas, eliminando assim os efeitos destas. Esta estrutura proporciona uma comparação mais precisa dos tratamentos do que nos experimentos em blocos ao acaso, com a desvantagem porém, de ser utilizado um número limitado de tratamentos, geralmente entre quatro e sete.

$O$ trabalho de WISHART \& SANDERS

recomenda que se faça com que o número de graus de liberdade do resíduo seja superior a 10 , o que permite quadrados latinos $3 \times 3$ e $4 \times 4$, ou seja, utilizar experimentos com menos de 5 tratamentos, somente quando são repetidos, lado a lado, 2 ou mais quadrados latinos de mesma natureza. 
Esses autores salientam ainda que deve haver um limite superior para o número de tratamentos que devem ser acomodados dentro da estrutura do quadrado latino pois, com mais de 7 ou 8 , as linhas e colunas tendem a ser muito compridas, comprometendo a eficiência do plano. Nestes casos, o autor comenta que não é aconselhável dividir os tratamentos em 2 ou mais grupos, cada qual em um quadrado latino, a não ser que se tenha o cuidado de incluir em todos os grupos, ao menos um controle ou tratamento padrão. Mesmo assim, a comparação entre os grupos não tem a mesma exatidão daquela dentro dos grupos.

Alguns trabalhos na área agronômica, como o de PATERSON (1946), citam que quando o experimento se estende sobre uma área considerável de terreno, a disposição em quadrado latino é mais vantajosa porque reduz o erro devido aos gradientes de fertilidade do solo.

FEDERER (1955), além do quadrado latino simples e do caso de grupos de quadrados latinos com uma observação por unidade experimental, apresenta em seu trabalho o caso de mais de uma observação por unidade experimental e fornece como exemplo um caso $4 \times 4$ com 6 observações por parcela (representadas por $\mathrm{d}=2$ leituras feitas em $\mathrm{p}=3$ plantas de feijão), considerando horas do dia como linhas, dias da semana como colunas e intensidades de luz como tratamentos.

Para este caso, o autor expressa a ijhgf-ésima observação como uma soma de vários efeitos independentes através do modelo: 


$$
\mathrm{X}_{\mathrm{ijhgf}}=\mu+\rho_{\mathrm{i}}+\lambda_{\mathrm{j}}+\tau_{\mathrm{h}}+\varepsilon_{\mathrm{ijh}}+\pi_{\mathrm{ijhg}}+\delta_{\mathrm{jhgf}}
$$

onde; genericamente :

$\mu$ é a média da população;

$\rho_{\mathrm{i}}$ é o efeito da i-ésima linha;

$\lambda_{\mathrm{j}}$ é o efeito da j-ésima coluna ;

$\tau_{\mathrm{h}}$ é o efeito do h-ésimo tratamento;

$\varepsilon_{\mathrm{ijh}}$ é o efeito da ijh-ésima parcela;

$\pi_{\mathrm{ijhg}}$ é o efeito da g-ésima planta na ijh-ésima parcela ;

$\delta_{\text {ijhgf }}$ é o efeito da ijhgf-ésima determinação;

$$
(\mathrm{i}, \mathrm{j}, \mathrm{h}=1,2, \ldots, \mathrm{k} ; \mathrm{g}=1,2, \ldots, \mathrm{p} ; \mathrm{f}=1,2, \ldots, \mathrm{d})
$$

e no exemplo $k=4, p=3$ e $d=2$.

O autor comenta que a hipótese de interesse é a da igualdade de efeitos dos níveis de tratamentos e fornece o seguinte esquema de análise de variância com os respectivos componentes de variância : 
Quadro 1. Esquema de análise da variância para experimentos em quadrado latino com 6 observações por casela, com as esperanças dos quadrados médios, segundo FEDERER (1955).

\begin{tabular}{ccc}
\hline Causas de Variação & G.L. & \multicolumn{1}{c}{$\mathrm{E}(\mathrm{Q} . \mathrm{M})}$. \\
\hline $\begin{array}{c}\text { Linhas (horas do } \\
\text { dia) }\end{array}$ & $\mathrm{k}-1=3$ & $\sigma_{\delta}^{2}+\mathrm{d} \sigma_{\pi}^{2}+\mathrm{dp} \sigma_{\varepsilon}^{2}+\mathrm{kdp} \sigma_{\rho}^{2}$ \\
$\begin{array}{c}\text { Colunas (dias da } \\
\text { semana) }\end{array}$ & $\mathrm{k}-1=3$ & $\sigma_{\delta}^{2}+\mathrm{d} \sigma_{\pi}^{2}+\mathrm{dp} \sigma_{\varepsilon}^{2}+\mathrm{kdp} \sigma_{\lambda}^{2}$ \\
$\begin{array}{c}\text { Tratamentos } \\
\text { (intensidade de luz) }\end{array}$ & $\mathrm{k}-1=3$ & $\sigma_{\delta}^{2}+\mathrm{d} \sigma_{\pi}^{2}+\mathrm{dp} \sigma_{\varepsilon}^{2}+\mathrm{kdp} \sigma_{\tau}^{2}$ \\
$\begin{array}{c}\text { Erro Experimental } \\
\text { Entre plantas na } \\
\text { parcela }\end{array}$ & $\mathrm{k}^{2}(\mathrm{p}-1)(\mathrm{k}-2)=6$ & $\sigma_{\delta}^{2}+\mathrm{d} \sigma_{\pi}^{2}+\mathrm{dp} \sigma_{\varepsilon}^{2}$ \\
Entre Leituras na & $\mathrm{pk}^{2}(\mathrm{~d}-1)=48$ & $\sigma_{\delta}^{2}+\mathrm{d} \sigma_{\pi}^{2}$ \\
mesma planta & & \\
\hline Total & $\mathrm{dpk}^{2}-1=95$ & \\
\hline
\end{tabular}

OSTLE (1956), além da análise simples de quadrado latino tambem apresenta, superficialmente, o caso de perda de uma observação. $O$ mesmo autor mostra tambem o caso de $m$ tratamentos com $n$ observações por unidade experimental, onde n é o mesmo para todas as parcelas. Neste caso, o esquema da análise da variância apresentado assemelha-se ao de FEDERER (1955) e é dado por : 
Quadro 2. Esquema de análise da variância para experimentos em quadrado latino com $n$ observações por unidade experimental, segundo OSTLE (1956).

\begin{tabular}{lccc}
\hline Causas de Variação & G.L. & S.Q. & Q.M. \\
\hline Linhas & $\mathrm{m}-1$ & Ryy & Ryy $/(\mathrm{m}-1)$ \\
Colunas & $\mathrm{m}-1$ & Cyy & Cyy $/(\mathrm{m}-1)$ \\
Tratamentos & $\mathrm{m}-1$ & Tyy & Tyy $/(\mathrm{m}-1)$ \\
Erro Experimental & $(\mathrm{m}-1)(\mathrm{m}-2)$ & Eyy & Eyy $/(\mathrm{m}-1)(\mathrm{m}-2)$ \\
Erro Amostral & $\mathrm{m}^{2}(\mathrm{n}-1)$ & Syy & $\mathrm{Syy} / \mathrm{m}^{2}(\mathrm{n}-1)$ \\
\hline Total & $\mathrm{m}^{2} \mathrm{n}-1$ & $\sum \mathrm{y}^{2}$ & \\
\hline
\end{tabular}

com

$$
\text { Ryy }=\frac{\sum_{i=1}^{m} R_{i}^{2}}{m}-\frac{T^{2}}{m^{2}} \quad ; \quad \text { Cyy }=\frac{\sum_{j=1}^{m} C_{j}^{2}}{m}-\frac{T^{2}}{m^{2}} \quad ; \quad \text { Tyy }=\frac{\sum_{k=1}^{m} T_{k}^{2}}{m}-\frac{T^{2}}{m^{2}} ;
$$

onde

T é o total de todas as observações;

$R_{i}, C_{j}$ e $T_{k}$ são, respectivamente, os totais da i-ésima linha, da j-ésima coluna e do k-ésimo tratamento;

$$
\begin{aligned}
& \text { Eyy }=\sum \mathrm{y}^{2}-\text { Ryy }- \text { Cyy }- \text { Tyy }- \text { Syy } \\
& \text { Syy }=\sum \mathrm{y}^{2}-\text { Ayy } \\
& \text { Ayy }=\text { Eyy }+ \text { Ryy }+ \text { Cyy }+ \text { Tyy }
\end{aligned}
$$


$\mathrm{O}$ autor salienta que o teste $\mathrm{F}$ apropriado para testar a hipótese $\mathrm{H}_{0}: \tau_{\mathrm{k}}=0,(\mathrm{k}=1,2, \ldots, \mathrm{m})$, é dado por :

$$
\mathrm{F}=\frac{\mathrm{QM} \text { Tratamentos }}{\text { QM Erro Experimental }}=\frac{\text { Tyy } /(\mathrm{m}-1)}{\text { Eyy } /(\mathrm{m}-1)(\mathrm{m}-2)} .
$$

No texto em questão, são apresentados ainda vários exemplos de aplicação do quadrado latino na experimentação com plantas, dentre eles são testados fertilizantes em cana-de-açúcar, em batata, em milho e em cacau. No caso de uso de fertilizantes em cacau é apresentado um exemplo de quadrado latino $3 \times 3$ repetido 3 vezes.

Alguns autores, como COCHRAN \& COX (1957) e KEMPTHORNE (1975) salientam que os quadrados latinos são muito usados na agricultura e têm em sua área experimental $\mathrm{k}^{2}$ parcelas, onde $\mathrm{k}$ é o número de tratamentos, de linhas e de colunas. Esses autores apresentam planos para $\mathrm{k}=3,4, \ldots, 12 \mathrm{e}$ comentam que esta estrutura experimental não afeta a precisão com que as médias de tratamentos são comparadas. Estes autores apresentam ainda os casos em que há perda de uma parcela, de uma linha, de uma coluna ou de um tratamento e salientam que a dificuldade na análise aumenta muito quando ocorre alguma perda de observação.

O trabalho de COCHRAN \& COX (1957) salienta a importância do quadrado latino na indústria, quando freqüentemente existem problemas como máquinas, períodos, operadores, etc , a serem controlados para estudar os tratamentos de interesse. O mesmo trabalho apresenta um caso interessante, onde as linhas não podem, por algum motivo, no 
planejamento, ser colocadas uma ao lado da outra. Esta limitação induz o experimentador a colocar as unidades experimentais em uma linha contínua, conforme o esquema a seguir:

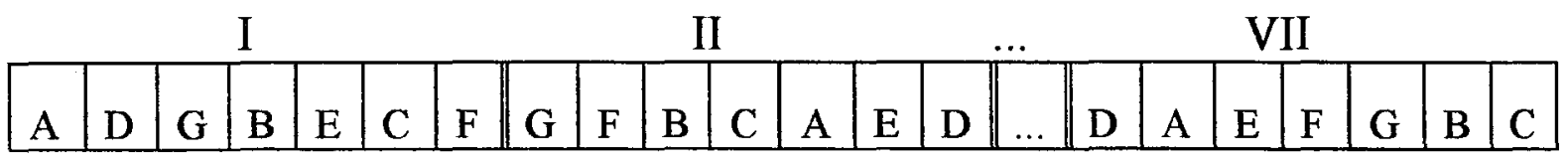

$\mathrm{O}$ autor também apresenta o caso de quadrado latino de ordem pequena $(2,3$ e 4$)$ com repetição ou, como alguns autores denominam, quadrados latinos múltiplos.

O quadrado latino é apresentado por SCHEFFÉ (1959) como um plano incompleto de classificação tripla no qual os três fatores têm o mesmo número $\mathrm{m}$ de níveis e as observações são tomadas em somente $\mathrm{m}^{2}$ das $\mathrm{m}^{3}$ combinações de tratamentos possíveis. $\mathrm{O}$ autor fala que a vantagem do quadrado latino sobre a classificação tripla completa é a de que somente $1 / \mathrm{m}$ combinações são necessárias e que a grande desvantagem é a de que, se houver interação entre os fatores, a análise estará comprometida.

$\mathrm{O}$ autor mostra também o processo de casualização e salienta que, para um quadrado $\mathrm{m}^{2}$ na forma padrão, podem-se obter $\mathrm{m} !(\mathrm{m}-1)$ ! quadrados fazendo $\mathrm{m}$ ! permutações nas colunas e (m-1)! nas linhas, deixando a primeira linha sem permuta. Procedimento semelhante é apresentado por OGAWA (1974). 
GRAYBILL (1961) discute o caso de classificação cruzada com $n$ fatores sem interação. Tomando o caso de $n=4$, o autor admite o seguinte modelo :

$$
\mathrm{y}_{\mathrm{ijkm}}=\mu+\alpha_{\mathrm{i}}+\beta_{\mathrm{j}}+\gamma_{\mathrm{k}}+\tau_{\mathrm{m}}+\mathrm{e}_{\mathrm{ijkm}}
$$

onde :

$\mu$ é a média geral da população ;

$\alpha_{\mathrm{i}}$ é o efeito do i-ésimo nível do fator $\mathrm{A}$;

$\beta_{\mathrm{j}}$ é o efeito do j-ésimo nível do fator $\mathrm{B}$;

$\gamma_{\mathrm{k}}$ é o efeito do k-ésimo nível do fator $\mathrm{C}$;

$\tau_{\mathrm{m}}$ é o efeito do m-ésimo nível do fator $\mathrm{D}$;

$\mathrm{e}_{\mathrm{j} \mathrm{jkm}}$ é o erro experimental .

O esquema para a análise da variância apresentado pelo autor é : 
Quadro 3. Esquema de análise da variância para o caso de modelo com classificação cruzada com $n=4$ fatores sem interação, segundo GRAYBILL (1961).

\begin{tabular}{|c|c|c|c|c|}
\hline Causas de Variação & G.L. & S.Q. & Q.M. & $E(Q . M)$. \\
\hline Total & pqrs & $\sum_{\mathrm{ijkm}} \mathrm{y}_{\mathrm{i} j \mathrm{~km}}^{2}$ & & \\
\hline Média & 1 & $\frac{\mathrm{y}_{\mathrm{ijkm}}^{2}}{\mathrm{pqrs}}$ & & \\
\hline$\alpha$ (ajustado) & $\mathrm{p}-1$ & $\alpha_{\mathrm{SQ}}$ & $\alpha_{\mathrm{QM}}$ & $\sigma^{2}+\frac{q r s}{p-1} \sum_{i}\left(\alpha_{i}-\bar{\alpha}\right)^{2}$ \\
\hline$\beta$ (ajustado) & $q-1$ & $\beta_{\mathrm{SQ}}$ & $\beta_{\mathrm{QM}}$ & $\sigma^{2}+\frac{p r s}{q-1} \sum_{j}\left(\beta_{j}-\bar{\beta}\right)^{2}$ \\
\hline$\gamma$ (ajustado) & $\mathrm{r}-1$ & $\gamma_{\mathrm{sQ}}$ & $\gamma_{\mathrm{QM}}$ & $\sigma^{2}+\frac{\mathrm{pqs}}{\mathrm{r}-1} \sum_{\mathrm{k}}\left(\gamma_{\mathrm{k}}-\bar{\gamma}\right)^{2}$ \\
\hline$\tau$ (ajustado) & $\mathrm{s}-1$ & $\tau_{\mathrm{SQ}}$ & $\tau_{\mathrm{QM}}$ & $\sigma^{2}+\frac{p q r}{s-1} \sum_{m}\left(\tau_{m}-\bar{\tau}\right)^{2}$ \\
\hline Resíduo & $\begin{array}{c}\text { pqrs-p-q-r- } \\
\mathrm{s}+3 \\
\end{array}$ & $\mathrm{E}_{\mathrm{SQ}}$ & $\mathrm{E}_{\mathrm{QM}}$ & $\sigma^{2}$ \\
\hline
\end{tabular}

As hipóteses testadas pelo autor são :
(1) $\alpha_{1}=\alpha_{2}=\ldots=\alpha_{p}$
(2) $\beta_{1}=\beta_{2}=\ldots=\beta_{q}$ 

(3) $\gamma_{1}=\gamma_{2}=\cdots=\gamma_{r}$
(4) $\tau_{1}=\tau_{2}=\cdots=\tau_{\mathrm{s}}$.

CHAKRABARTI (1962) apresenta toda a análise de variância para quadrado latino com uma observação por casela, através da teoria de modelos lineares, mostrando também o caso de parcelas subdivididas em quadrado latino.

Um procedimento simples para a análise do quadrado latino, bem como a forma de comparar as médias dos tratamentos é mostrado por autores como PANSE \& SUKHATME (1963) e GOMES (1978).

Um estudo sobre a análise de experimentos com parcelas perdidas é apresentado por CAMPOS (1964), determinando fórmulas para o cálculo das estimativas das parcelas perdidas e para as variâncias dos contrastes entre dois tratamentos, com e sem parcela perdida, em delineamento em blocos casualizados, em quadrados latinos e em períodos sucessivos.

Os trabalhos de VAJDA (1967) e RAGHAVARAO (1971) apresentam a estrutura matemática do delineamento em quadrado latino, mostrando as formas padrão e os arranjos ortogonais dessa estrutura, não se preocupando com a análise estatística desse delineamento.

Como a maior parte dos autores, o trabalho de SNEDECOR \& COCHRAN (1967) mostra o caso simples de quadrado latino com uma observação por unidade experimental, adotando o seguinte modelo matemático: 


$$
\begin{gathered}
\mathrm{x}_{\mathrm{ijk}}=\mu+\alpha_{\mathrm{i}}+\beta_{\mathrm{j}}+\gamma_{\mathrm{k}}+\varepsilon_{\mathrm{ijk}} ; \\
\left(\mathrm{i}, \mathrm{j} \text { e k }=1, \ldots, \mathrm{a} ; \varepsilon_{\mathrm{ijk} k} \cap \mathrm{N}\left(0, \sigma^{2}\right)\right) ;
\end{gathered}
$$

onde $\alpha, \beta$ e $\gamma$ indicam efeitos de tratamentos, linhas e colunas, respectivamente.

Os autores apresentam, também, o caso de uma parcela perdida, e fornecem uma regra para sua estimação.

O trabalho de KIRK (1968), com experimentos em quadrados latinos $4 \times 4$ visa avaliar o desempenho de quatro tipos de pneus de automóveis considerados como tratamentos, tomando modelos de automóveis, como linhas e posições das rodas como colunas. Além disto, os testes são repetidos duas vezes, considerando duas leituras por casela, constituindo-se assim num caso particular do estudo de OSTLE (1956) citado anteriormente.

Em seu trabalho, o autor faz várias considerações sobre o delineamento em quadrado latino, dentre elas, que o quadrado latino de ordem menor que 5 , com uma observação por casela não é muito usual na prática devido ao baixo número de graus de liberdade do resíduo. $\mathrm{O}$ autor sugere, como alternativa, que sejam usados vários quadrados latinos repetidos ou que se tenha mais de uma observação por casela em um único deles.

Para o caso de mais de uma observação por casela o autor considera:

p níveis de linha (fator $\mathrm{A})$; 
$q$ níveis de coluna (fator $B$ ) ;

$\mathrm{r}$ níveis de tratamento (fator $\mathrm{C}$ );

n observações por casela, $\operatorname{com} p=q=r$.

Nesse caso, admite o modelo:

$$
\mathrm{X}_{\mathrm{ijkm}}=\mu+\alpha_{\mathrm{i}}+\beta_{\mathrm{j}}+\gamma_{\mathrm{k}}+\text { residuo }+\varepsilon_{\mathrm{m}(\mathrm{ijk})} ;
$$

onde:

$\mathrm{X}_{\mathrm{ijkm}}$ é a medida da ijkm-ésima observação;

$\mu$ é a média geral ;

$\alpha_{i}$ é o efeito da i-ésima linha , $\sum_{i=1}^{p} \alpha_{i}=0$;

$\beta_{\mathrm{j}}$ é o efeito da $\mathrm{j}$-ésima coluna,$\sum_{\mathrm{j}=1}^{\mathrm{q}} \beta_{\mathrm{j}}=0$;

$\gamma_{\mathrm{k}}$ é o efeito do k-ésimo tratamento, $\sum_{\mathrm{k}=1}^{\mathrm{r}} \gamma_{\mathrm{k}}=0$;

resíduo é todo efeito devido aos níveis de linhas, de colunas e de tratamentos não previstos pela soma desses efeitos. Se toda interação entre esses efeitos é zero, então a média do resíduo é zero com variância $\sigma_{\text {res. ; }}^{2}$

$\varepsilon_{\mathrm{m}(\mathrm{ijk})}$ é o erro experimental, independente e normalmente distribuído em cada casela, com média zero e variância $\sigma_{\varepsilon}^{2}$; 
O esquema de análise da variância, com as esperanças dos quadrados médios apresentado pelo autor é semelhante ao de FEDERER (1955) e ao de OSTLE (1956) e é dado por :

Quadro 4. Esquema de análise da variância para experimentos em quadrado latino com $\mathbf{n}$ observações por unidade experimental, com as esperanças dos quadrados médios, segundo KIRK (1968).

\begin{tabular}{lcl}
\hline Causas de Variação & G.L. & \multicolumn{1}{c}{ E(Q.M.) } \\
\hline Entre níveis de A & $\mathrm{p}-1$ & $\sigma_{\varepsilon}^{2}+n p \sigma_{\alpha}^{2}$ \\
Entre níveis de B & $\mathrm{p}-1$ & $\sigma_{\varepsilon}^{2}+n p \sigma_{\beta}^{2}$ \\
Entre níveis de C & $\mathrm{p}-1$ & $\sigma_{\varepsilon}^{2}+n p \sigma_{\gamma}^{2}$ \\
Resíduo & $(\mathrm{p}-1)(\mathrm{p}-2)$ & $\sigma_{\varepsilon}^{2}+n \sigma_{\text {res. }}^{2}$ \\
Dentro de caselas & $\mathrm{p}^{2}(\mathrm{n}-1)$ & $\sigma_{\varepsilon}^{2}$ \\
\hline Total & $\mathrm{np} \mathrm{p}^{2}-1$ & \\
\hline
\end{tabular}

Para a razão $\mathrm{F}$ o autor toma o modelo de efeitos fixos e a hipótese testada é :

$$
\begin{aligned}
& \mathrm{H}_{0}: \gamma_{\mathrm{k}}=0, \text { para todo } \mathrm{k}, \\
& \mathrm{H}_{1}: \gamma_{\mathrm{k}} \neq 0 \text {, para algum } \mathrm{k},
\end{aligned}
$$

onde $\mathrm{k}$ é o índice de tratamentos . 
O autor comenta ainda que a aplicação clássica do quadrado latino na pesquisa agrícola envolve o tratamento de interesse e duas variáveis "nuisance" que são as linhas e as colunas. Se o quadrado latino contém dois tratamentos e uma variável "nuisance" ele é o chamado delineamento quadrado latino em fatorial fracionado. Se ambas as variáveis designadas por linhas e colunas representam tratamentos ao invés de variáveis "nuisance" , o delineamento é descrito como um fatorial fracionado.

Assim, o autor mostra, como exemplo, o quadrado latino padrão dado por :

\begin{tabular}{l|l|l|l|}
\multicolumn{1}{c}{} & \multicolumn{1}{c}{$\mathrm{b}_{0}$} & \multicolumn{1}{c}{$\mathrm{b}_{1}$} & \multicolumn{1}{c}{$\mathrm{b}_{2}$} \\
\cline { 2 - 4 } $\mathrm{a}_{0}$ & $\mathrm{c}_{0}$ & $\mathrm{c}_{1}$ & $\mathrm{c}_{2}$ \\
\cline { 2 - 4 } $\mathrm{a}_{1}$ & $\mathrm{c}_{1}$ & $\mathrm{c}_{2}$ & $\mathrm{c}_{0}$ \\
\cline { 2 - 4 } $\mathrm{a}_{2}$ & $\mathrm{c}_{2}$ & $\mathrm{c}_{0}$ & $\mathrm{c}_{1}$ \\
\cline { 2 - 4 } & & &
\end{tabular}

onde $a_{i}, b_{j}$ e $c_{k}$ representam os 3 tratamentos e as combinações desses tratamentos são dadas por :

$$
\begin{aligned}
\left(\mathrm{ABC}^{2}\right) & =\left[\mathrm{x}_{1}+\mathrm{x}_{2}+2 \mathrm{x}_{3}=0(\text { mod. } 3)\right] \\
& =000,011,022,101,112,120,202,210,221 .
\end{aligned}
$$

Desta forma, um quadrado latino padrão $3 \times 3$ corresponde a qualquer $1 / 3$ das combinações possíveis de um experimento fatorial $3^{3}$, 
ou seja, o fatorial completo contém 27 combinações enquanto que o quadrado latino contém somente 9 dessas combinações.

JOHN (1971) cita um exemplo de quadrado latino $5 \times 5$ com carros, onde testa diferentes tipos de gasolina, bloqueando o efeito de dias e de carros, ou seja, usando dias da semana como linhas e os carros como colunas. No seu trabalho encontra-se ainda, como no de outros autores, o caso de quadrados latinos repetidos. $\mathrm{O}$ autor também salienta a importância do quadrado latino na experimentação com animais, dando ênfase para o caso de experimentos com vacas.

WINNER (1971), dos autores, é o que apresenta maior grau de detalhamento dos experimentos em quadrado latino. Descreve a estrutura, a aleatorização, a análise simples e os casos particulares como o de medidas repetidas e o de medidas não repetidas, onde as observações são obtidas de indivíduos distintos em uma mesma casela. Para o caso de medidas não repetidas, se encaixa o caso de quadrado latino com mais de uma observação por unidade experimental. Da mesma forma que OSTLE (1956) e KIRK (1968), o autor apresenta somente o caso de quadrado latino com mais de uma observação por unidade experimental quando o número de observações em todas as caselas é o mesmo.

O modelo adotado por WINNER (1971), para o caso de mais de uma observação por casela, sendo o número de observações sempre o mesmo em todas as caselas é dado por : 


$$
\mathrm{X}_{\mathrm{ijkm}}=\mu+\alpha_{\mathrm{i}(\mathrm{s})}+\beta_{\mathrm{j}(\mathrm{s})}+\gamma_{\mathrm{k}(\mathrm{s})}+\operatorname{res}_{(\mathrm{s})}+\varepsilon_{\mathrm{m}(\mathrm{jkk})}
$$

onde $\alpha, \beta$ e $\gamma$ indicam efeitos de tratamentos, linhas e colunas e o subscrito (s) indica que os dados são de um determinado quadrado latino (s) . $\mathrm{O}$ esquema de análise da variância apresentado no trabalho é :

Quadro 5. Esquema de análise da variância para experimentos em quadrado latino, com número igual de observações por unidade experimental, segundo WINNER (1971).

\begin{tabular}{lcl}
\hline Causas de Variação & G.L. & E(Q.M.) \\
\hline A & $\mathrm{p}-1$ & $\sigma_{\varepsilon}^{2}+n p \sigma_{\alpha}^{2}$ \\
B & $\mathrm{p}-1$ & $\sigma_{\varepsilon}^{2}+n p \sigma_{\beta}^{2}$ \\
C & $\mathrm{p}-1$ & $\sigma_{\varepsilon}^{2}+n p \sigma_{\dot{\gamma}}^{2}$ \\
Resíduo & $(\mathrm{p}-1)(\mathrm{p}-2)$ & $\sigma_{\varepsilon}^{2}+n \sigma_{\text {res }}^{2}$ \\
Dentro de caselas & $\mathrm{p}^{2}(\mathrm{n}-1)$ & $\sigma_{\varepsilon}^{2}$ \\
\hline Total & $\mathrm{np} \mathrm{p}^{2}-1$ \\
\hline
\end{tabular}

ANDERSON \& MCLEAN (1974) consideram o quadrado latino como um excelente delineamento quando o objetivo é remover causas de variação em duas direções e somente um conjunto de tratamentos tem interesse. Estes autores tomam o mesmo processo usado para blocos, com blocos fixos, assumem que todas as interações são zero e o modelo adotado é escrito como: 


$$
\begin{gathered}
y_{i j k}=\mu+R_{i}+\delta_{(i)}+C_{j}+\eta_{(j)}+T_{k}+\varepsilon_{(j \mathrm{jk})} \\
i=j=k=1,2, \ldots, t
\end{gathered}
$$

onde:

$\mathrm{y}_{\mathrm{ijk}}$ é a variável referente a i-ésima linha, j-ésima coluna e $\mathrm{k}$-ésimo tratamento ;

$\mu$ é a média geral ;

$R_{i}$ é o efeito da i-ésima linha;

$\delta_{(i)}$ é o erro na linha, devido à restrição na casualização dos tratamentos na i-ésima linha ;

$\mathrm{C}_{\mathrm{j}}$ é o efeito da j-ésima coluna ;

$\eta_{(j)}$ é o erro na coluna, devido a restrição na casualização dos tratamentos na j-ésima coluna ;

$\mathrm{T}_{\mathrm{k}}$ é o efeito do k-ésimo tratamento;

$\varepsilon_{(\mathrm{ijk})}$ é o erro experimental para a i-ésima linha, j-ésima coluna e k-ésimo tratamento, estimado pelo resíduo .

O esquema de análise da variância correspondente é: 
Quadro 6. Esquema de análise da variância para experimentos em quadrado latino com as esperanças dos quadrados médios, segundo ANDERSON \& MCLEAN (1974).

\begin{tabular}{lcl}
\hline Causas de Variação & G.L. & \multicolumn{1}{c}{$E(\mathrm{Q} . \mathrm{M})}$. \\
\hline Linhas (R) & $\mathrm{t}-1$ & $\sigma^{2}+\mathrm{t \sigma}_{\delta}^{2}+\mathrm{t} \phi(\mathrm{R})$ \\
Erro pela restrição de linhas & 0 & $\sigma^{2}+\mathrm{t} \sigma_{\delta}^{2}$ \\
Colunas (C) & $\mathrm{t}-1$ & $\sigma^{2}+\mathrm{t} \sigma_{\eta}^{2}+\mathrm{t} \phi(\mathrm{C})$ \\
Erro pela restrição de colunas & 0 & $\sigma^{2}+\mathrm{t} \sigma_{\eta}^{2}$ \\
Tratamentos (T) & $\mathrm{t}-1$ & $\sigma^{2}+\mathrm{t} \phi(\mathrm{T})$ \\
Erro Experimental (Resíduo) & $(\mathrm{t}-1)(\mathrm{t}-2)$ & $\sigma^{2}$ \\
\hline Total & $\mathrm{t}^{2}-1$ & \\
\hline
\end{tabular}

A hipótese de interesse para o autor é :

$$
\mathrm{H}_{0}: \mathrm{T}_{\mathrm{k}}=0 \text {, para todo } \mathrm{k} \text {. }
$$

O esquema de análise da variância apresentado por OGAWA (1974) para um quadrado latino de ordem $t$, onde considera o modelo de efeitos fixos, é : 
Quadro 7. Esquema de análise da variância para experimentos em quadrado latino com as esperanças dos quadrados médios, segundo OGAWA (1974).

\begin{tabular}{lccc}
\hline Causas de Variação & G.L. & S.Q. & E(Q.M.) \\
\hline Linhas & $\mathrm{t}-1$ & $\frac{1}{\mathrm{t}} \sum \mathrm{R}_{\mathrm{i}}^{2}-\mathrm{n} \overline{\mathrm{x}}^{2}$ & $\sigma^{2}+\frac{\mathrm{t}}{\mathrm{t}-1} \sum \pi_{\mathrm{i}}^{2}$ \\
Colunas & $\mathrm{t}-1$ & $\frac{1}{\mathrm{t}} \sum \mathrm{C}_{\mathrm{j}}^{2}-\mathbf{n} \overline{\mathrm{x}}^{2}$ & $\sigma^{2}+\frac{\mathrm{t}}{\mathrm{t}-1} \sum \gamma_{\mathrm{j}}^{2}$ \\
Tratamentos & $\mathrm{t}-1$ & $\frac{1}{\mathrm{t}} \sum \mathrm{T}_{\alpha}^{2}-\mathrm{n} \overline{\mathrm{x}}^{2}$ & $\sigma^{2}+\frac{\mathrm{t}}{\mathrm{t}-1} \sum \tau_{\alpha}^{2}$ \\
Erro & $(\mathrm{t}-1)(\mathrm{t}-2)$ & por subtração & $\sigma^{2}$ \\
\hline Total & $\mathrm{t}^{2}-1$ & $\sum \mathrm{x}_{\mathrm{f}}^{2}-\mathrm{nx}^{-2}$ & \\
\hline
\end{tabular}

Fica implícito que o autor tem interesse em testar a hipótese $\mathrm{H}_{0}: \tau_{\alpha}=0$, para todo $\alpha$.

Segundo NETER \& WASSERMAN (1974), além das considerações usuais sobre quadrado latino existe o caso de réplicas na casela, denotando $Y_{\mathrm{ij}(k) \mathrm{m}}$ como a observação da m-ésima unidade $(\mathrm{m}=1,2$, $\ldots, n)$ na parcela $(i, j)$ que recebe o k-ésimo tratamento.

O esquema da análise da variância apresentado nesse trabalho é: 
Quadro 8. Esquema de análise da variância para experimentos em quadrado latino com $\mathrm{n}$ observações por casela, segundo NETER \& WASSERMAN (1974).

\begin{tabular}{lcc}
\hline Causas de Variação & G.L. & S.Q. \\
\hline Linhas & $\mathrm{r}-1$ & S.Q.Linhas \\
Colunas & $\mathrm{r}-1$ & S.Q.Colunas \\
Tratamentos & $\mathrm{r}-1$ & S.Q.Tratamentos \\
Erro & $\mathrm{nr}^{2}-3 \mathrm{r}+2$ & S.Q.Erro \\
\multicolumn{1}{c}{ Falta de Ajuste } & $(\mathrm{r}-1)(\mathrm{r}-2)$ & S.Q.Falta de Ajuste \\
\multicolumn{1}{c}{ Erro Puro } & $(\mathrm{n}-1) \mathrm{r}^{2}$ & S.Q.Erro Puro \\
\hline Total & $\mathrm{nr}^{2}-1$ & S.Q.Total \\
\hline
\end{tabular}

onde :

$$
\begin{aligned}
& \text { S.Q.Total }=\sum_{i} \sum_{j} \sum_{m}\left(Y_{i j(k) m}-\bar{Y}_{\ldots . . .}\right)^{2}=\sum_{i} \sum_{j} \sum_{m} Y_{i j(k) m}^{2}-\frac{Y_{\ldots .}^{2}}{r^{2} n} \\
& \text { S.Q.Trat. }=\operatorname{rn} \sum_{k}\left(\bar{Y}_{. . k .}-\bar{Y}_{\ldots . .}\right)^{2}=\frac{\sum_{k} Y_{. . k .}^{2}}{r n}-\frac{Y_{\ldots .}^{2}}{r^{2} n} \\
& \text { S.Q. Linhas }=m \sum_{i}\left(\bar{Y}_{i . . .}-\bar{Y}_{\ldots .}\right)^{2} \equiv \frac{\sum_{i} Y_{i . . .}^{2}}{r n}-\frac{Y_{\ldots}^{2}}{r^{2} n} \\
& \text { S.Q.Colunas }=m \sum_{j}\left(\bar{Y}_{. j .}-\bar{Y}_{. . .}\right)^{2}=\frac{\sum_{j} Y_{. j .}^{2}}{m}-\frac{Y^{2}}{r^{2} n}
\end{aligned}
$$




$$
\begin{aligned}
& \text { S.Q.Erro = S.Q.Total - S.Q.Linhas - S.Q.Colunas - S.Q. Trat. } \\
& \text { S.Q.E.Puro }=\sum_{\mathrm{i}} \sum_{\mathrm{j}} \sum_{\mathrm{m}}\left(\mathrm{Y}_{\mathrm{ij}(\mathrm{k}) \mathrm{m}}-\overline{\mathrm{Y}}_{\mathrm{ij}(\mathrm{k}) .}\right)^{2}=\sum_{\mathrm{i}} \sum_{\mathrm{j}} \sum_{\mathrm{m}} \mathrm{Y}_{\mathrm{ij}(\mathrm{k}) \mathrm{m}}^{2}-\sum_{\mathrm{i}} \sum_{\mathrm{j}} \frac{\mathrm{Y}_{\mathrm{j} j(\mathrm{k})}^{2}}{\mathbf{n}} \\
& \text { S.Q.Falta de Ajuste = S.Q.Erro - S.Q.Erro Puro }
\end{aligned}
$$$$
\text { As hipóteses testadas nesse trabalho são : }
$$

$$
\begin{gathered}
\mathrm{C}_{1}: \text { todo } \tau_{\mathrm{k}}=0 \\
\mathrm{C}_{2}: \text { nem todo } \tau_{\mathrm{k}}=0
\end{gathered}
$$

No caso de medidas não repetidas, se encaixa o caso de quadrado latino com mais de uma observação por unidade experimental. Salienta-se no entanto que, da mesma forma que OSTLE (1956) e KIRK (1968), o autor apresenta o caso de quadrado latino com mais de uma observação por unidade experimental somente quando o número de observações por casela é constante.

LITTLE \& HILLS (1975) apresentam uma partição adicional onde a soma de quadrados de tratamentos e o número de graus de liberdade correspondente são desmembrados, caracterizando o que se denomina estrutura fatorial de tratamentos. Neste delineamento de tratamentos, os efeitos de dois ou mais fatores são pesquisados simultaneamente, de tal forma que os efeitos de um fator sobre o outro podem ser detectados e quantificados. Este tipo de partição tambem é apresentado por NETER \& WASSERMAN (1974). 
KEMPTHORNE (1975) apresenta o quadrado latino como um caso particular de experimento fatorial, onde a interação entre os fatores não tem interesse e o quadrado latino representa um grupo de confundimento.

O estudo de BUENO (1976) mostra um quadrado latino quando ocorre perda de duas observações em tratamentos, linhas e colunas diferentes, ou em uma mesma coluna, ou em uma mesma linha ou em um mesmo tratamento.

Além do delineamento, no qual se inclui o controle local através da formação de blocos, e pela forma com que os tratamentos ou combinações de tratamentos são distribuídos nas parcelas, PEARCE (1976) coloca o esquema de análise da variância onde a soma de quadrados total, e o respectivo número de graus de liberdade, desmembra-se em soma de quadrados de linhas, soma de quadrados de colunas, soma de quadrados de tratamentos e soma de quadrados de erro experimental.

O trabalho de LITTLE \& HILLS (1978) apresenta o uso de contrastes ortogonais para comparar médias desdobrando a soma de quadrados de tratamentos e mostra um exemplo agronômico de parcelas subdivididas com quadrado latino na parcela. No exemplo, os autores usam um quadrado latino $4 \times 4$, onde os tratamentos são 4 níveis de nitrogênio e como subtratamentos usam 5 épocas de colheita.

Estudando os experimentos em quadrado latino, JOHN (1971), MILLIKEN \& JOHNSON (1984), dentre outros, apresentam a análise de variância para a estrutura de dois tratamentos (fatores) em um delineamento em quadrado latino repetido. 
MILLIKEN \& JOHNSON (1984) são autores que se preocupam com a parte computacional da análise e usam o programa SAS em todo o texto, como o programa indicado para análise de dados desbalanceados.

Segundo GOMES (1984), o delineamento em quadrado latino não é muito usado na experimentação vegetal devido a sua pequena flexibilidade, ou seja, os números de tratamentos, de linhas e de colunas devem ser iguais. $\mathrm{O}$ autor comenta que este delineamento é comumente usado em ensaios com vacas leiteiras. No mesmo trabalho, o autor diz que a vantagem do delineamento é eliminar melhor a heterogeneidade do material experimental devido ao uso de linhas e de colunas e que uma desvantagem é a grande redução no número de graus de liberdade do resíduo, lembrando bem como LITTLE \& HILLS (1978), dentre outros, que este delineamento é usado em geral para 4 a 8 tratamentos.

Nos trabalhos iniciais com quadrado latino, nota-se uma preocupação dos autores em determinar o valor ou os valores de parcelas perdidas para que se possa levar avante a análise da variância. Dentre numerosos autores que trabalharam nesta área, temos SANCHES (1978), que apresenta uma boa revisão bibliográfica e complementa o assunto com o estudo da análise estatística de um experimento em quadrado latino $\mathrm{r} \times \mathrm{r}$, onde ocorrem perdas de uma parcela, de duas parcelas de tratamentos distintos, de duas parcelas de um mesmo tratamento, de um tratamento, de uma linha ou de uma coluna.

MURRAY (1986) através das fórmulas clássicas para estimar parcelas perdidas em delineamentos em blocos ao acaso e quadrado 
latino, faz sua proposta usando o modelo de médias de caselas e apresenta um exemplo para cada delineamento.

O manual do SAS (1990) recomenda, para o caso de quadrado latino com número igual de repetições por casela, o uso do SAS/PROC ANOVA para a análise da variância. Para os casos de desbalanceamento, esse trabalho indica o SAS/PROC GLM . Nota-se que, quanto aos pacotes estatísticos, somente os livros mais recentes, da área de estatística experimental e de modelos lineares têm a preocupação de citar alguns deles.

Uma discussão sobre modelos de classificação tripla cruzada é apresentada por SEARLE (1987), onde se pode encaixar o caso de quadrado latino. $\mathrm{O}$ autor discute o caso em que todas as caselas são ocupadas e também o caso de algumas caselas vazias. Todo seu trabalho está baseado na teoria de modelos lineares usando a notação $R($ ) .

SEARLE (1987) classifica o delineamento quadrado latino como um caso particular de desbalanceamento que denomina de desbalanceamento planejado e mostra, como exemplo, o seguinte caso :

\section{Tratamento A Tratamento B Tratamento C}

\begin{tabular}{c|c|c|c|c|c|c|c|c|c}
\multicolumn{9}{c|}{ Colunas } & \multicolumn{1}{c}{ Colunas } \\
\hline Linhas & 1 & 2 & 3 & 1 & 2 & 3 & 1 & 2 & 3 \\
\hline 1 & 1 & 0 & 0 & 0 & 1 & 0 & 0 & 0 & 1 \\
\hline 2 & 0 & 1 & 0 & 0 & 0 & 1 & 1 & 0 & 0 \\
\hline 3 & 0 & 0 & 1 & 1 & 0 & 0 & 0 & 1 & 0 \\
\hline
\end{tabular}


ou que é escrito ainda por :

\begin{tabular}{c|c|c|c}
\cline { 2 - 4 } Linhas & 1 & 2 & 3 \\
\hline 1 & A & B & C \\
\hline 2 & C & A & B \\
\hline 3 & B & C & A \\
\hline
\end{tabular}

Nesse trabalho, o autor comenta que em modelos compostos somente de efeitos principais, no caso balanceado, a hipótese testada por

$$
\mathrm{F}=\frac{\mathrm{R}(\text { fator } \alpha / \text { todos os outros fatores })}{(\mathrm{a}-1) \hat{\sigma}^{2}}
$$

é :

$\mathrm{H}_{0}$ : os efeitos de todos os niveis de $\alpha$ (tratamentos) são iguais .

Quanto às hipóteses testadas, alguns autores as apresentam de forma explícita. Alguns livros de modelos lineares, como GRAYBILL (1961) e SEARLE (1987) têm a preocupação em apresentar as hipóteses testadas para cada modelo, salientando que ocorrem alterações para modelos com interação e também para o caso de caselas vazias.

De acordo com JOHN (1987) se dois componentes do delineamento Dp e Dq são blocos casualizados, resultando o esquema linhacoluna, o delineamento é chamado quadrado latino. $O$ autor, dentre outros, apresenta o seguinte esquema de análise da variância para um quadrado latino com $\mathrm{v}$ tratamentos : 
Quadro 9. Esquema de análise da variância para experimentos em quadrado latino, segundo JOHN (1987).

\begin{tabular}{lcc}
\hline Causas de Variação & G.L. & S.Q. \\
\hline Dentro de Linhas & $\mathrm{v}-1$ & $(1 / \mathrm{v}) \mathrm{R} \cdot \mathrm{R}-\mathrm{G}^{2} / \mathrm{n}$ \\
Dentro de Colunas & $\mathrm{v}-1$ & $(1 / \mathrm{v}) \mathrm{C}^{\prime} \mathrm{C}-\mathrm{G}^{2} / \mathrm{n}$ \\
Dentro de Tratamentos & $\mathrm{v}-1$ & $(1 / \mathrm{v}) \mathrm{T}^{\prime} \mathrm{T}-\mathrm{G}^{2} / \mathrm{n}$ \\
Resíduo & $(\mathrm{v}-1)(\mathrm{v}-2)$ & por diferença \\
\hline Total & $\mathrm{n}-1$ & $\mathrm{y}^{\prime} \mathrm{y}-\mathrm{G}^{2} / \mathrm{n}$ \\
\hline
\end{tabular}

onde $\mathrm{R}, \mathrm{C}, \mathrm{T}$ e $\mathrm{G}$ são, respectivamente, os totais de linhas, colunas, tratamentos e geral das parcelas e $\mathbf{n}$ o número de parcelas. A hipótese testada através do teste $\mathrm{F}$ é a de igualdade de tratamentos .

STREET \& STREET (1987), assim como RIBOLDI (1988) e outros autores, mostram o quadrado latino como um caso particular de ensaios em blocos incompletos parcialmente balanceados. Esse trabalho é um dos poucos que apresenta esquemas de quadrados latinos de ordem superior a nove. Além desse caso, os autores dão exemplos de alguns outros delineamentos no esquema linha-coluna, como o quadrado de Youden e látices quadrados.

O trabalho de HEIBERGER (1989) mostra o delineamento quadrado latino, primeiramente na forma simples, como a maior parte dos autores, citando um exemplo de COCHRAN \& COX (1957), onde considera amostradores como linhas, áreas como colunas e 
testa tratamentos em trigo. Assim como GILL (1981), o autor discute o delineamento como um caso do delineamento fatorial com confundimento e ainda como um delineamento em blocos incompletos. $\mathrm{O}$ autor, no mesmo trabalho, cita programas como SAS, GENSTAT, P-STAT e GLIM para a análise de dados experimentais .

CAMPOS \& PIEDADE (1992) mostram alguns exemplos práticos de aplicação dos experimentos em quadrado latino na experimentação agronômica. O modelo matemático e o respectivo esquema de análise da variância apresentado neste trabalho são :

$$
\mathbf{x}_{\mathrm{ijk}}=\mathrm{m}+\mathrm{t}_{\mathrm{i}}+\mathrm{l}_{\mathrm{j}}+\mathrm{c}_{\mathrm{k}}+\mathrm{e}_{\mathrm{ijk}}
$$

onde:

$\mathrm{x}_{\mathrm{ijk}}$ é a observação do tratamento $\mathrm{i}$, na linha $\mathrm{j}$ e na coluna $\mathrm{k}$;

m é a média geral ;

$\mathrm{t}_{\mathrm{i}}$ é o efeito do tratamento $\mathrm{i}$;

$1_{\mathrm{j}}$ é o efeito da linha $\mathrm{j}$;

$c_{k}$ é o efeito da coluna $\mathrm{k}$;

$\mathrm{e}_{\mathrm{ijk}}$ é o erro da parcela ijk; 
Quadro 10. Esquema de análise da variância para experimentos em quadrado latino, segundo CAMPOS \& PIEDADE (1992).

\begin{tabular}{lc}
\hline Causas de Variação & G.L. \\
\hline Tratamentos & $\mathrm{K}-1$ \\
Linhas & $\mathrm{K}-1$ \\
Colunas & $\mathrm{K}-1$ \\
Resíduo & $(\mathrm{K}-1)(\mathrm{K}-2)$ \\
\hline Total & $\mathrm{K}^{2}-1$ \\
\hline
\end{tabular}

Tais esquemas são semelhantes aos apresentados por outros autores, dentre eles, COCHRAN \& COX (1957), PANSE \& SUKHATME (1963).

Num trabalho mais recente, FEDERER (1993), salienta que existem vários tipos de delineamentos de r-linhas por c-colunas e cita alguns deles, dentre os quais o mais conhecido é o quadrado latino cujo modelo adotado é :

$$
\mathrm{y}_{\mathrm{hij}}=\mu+\rho_{\mathrm{h}}+\gamma_{\mathrm{i}}+\tau_{\mathrm{j}}+\varepsilon_{\mathrm{hij}}
$$

onde:

$\mathrm{y}_{\text {hij }}$ é a resposta do j-ésimo tratamento na h-ésima linha e i-ésima coluna ;

$\mu$ é um efeito médio ;

$\rho_{\mathrm{h}}$ é o efeito da h-ésima linha ; 
$\gamma_{i}$ é o efeito da i-ésima coluna ;

$\tau_{\mathrm{j}}$ é o efeito do j-ésimo tratamento ;

$\varepsilon_{\mathrm{hij}}$ é o erro aleatório, independente e identicamente distribuído $\left(0, \sigma_{\varepsilon}^{2}\right)$.

O esquema de análise da variância é :

Quadro 11. Esquema de análise da variância para experimentos em quadrado latino, segundo FEDERER (1993).

\begin{tabular}{lcc}
\hline Causas de Variação & G.L. & S.Q. \\
\hline Total & rc & $\sum_{\mathrm{h}} \sum_{\mathrm{i}} \mathrm{y}_{\mathrm{hij}}^{2}$ \\
Correção para média & 1 & $\frac{\mathrm{y}_{\ldots .}^{2}}{\mathrm{rc}}$ \\
Colunas & $\mathrm{c}-1$ & $\frac{\sum_{\mathrm{i}} \mathrm{y}_{\mathrm{i} .}^{2}}{\mathrm{r}}-\frac{\mathrm{y}_{\ldots .}^{2}}{\mathrm{rc}}$ \\
Linhas & $\mathrm{r}-1$ & $\frac{\sum_{\mathrm{h}} \mathrm{y}_{\mathrm{h} .}^{2}}{\mathrm{c}}-\frac{\mathrm{y}_{. . .}^{2}}{\mathrm{rc}}$ \\
Tratamentos & $\mathrm{v}-1$ & $\mathrm{v} \sum_{\mathrm{j}} \mathrm{y}_{. . \mathrm{j}}^{2}-\mathrm{y}_{\ldots . .}^{2}$ \\
& & $\frac{\mathrm{rc}}{\mathrm{por} \mathrm{subtração}}$ \\
Resíduo & $(\mathrm{r}-1)(\mathrm{c}-1)-(\mathrm{v}-1)$ & \\
\hline
\end{tabular}


As hipóteses testadas nesse trabalho são :

$$
\begin{aligned}
& H_{0}: \tau_{j}=0 \text { para todo } j \\
& H_{a}: \text { pelo menos um } \tau_{j} \neq 0
\end{aligned}
$$




\section{DESENVOLVIMENTO TEÓRICO}

\subsection{Introdução}

Para o desenvolvimento deste trabalho admite-se um ensaio em quadrado latino, com $\mathrm{K}$ tratamentos, I linhas e $\mathrm{J}$ colunas, onde, devido à própria característica do delineamento $\mathrm{I}=\mathrm{J}=\mathrm{K}$. Considera-se também, que o experimento tem $\mathrm{r}=1,2, \ldots, \mathrm{n}_{\mathrm{ijk}}$ observações por unidade experimental.

Apresenta-se, inicialmente, como exemplo, um caso em que $\mathrm{I}=\mathrm{J}=\mathrm{K}=3$; assim, esquematicamente, tem-se : 
Quadro 12. Esquema de um quadrado latino $3 \times 3$ com número diferente de observações por unidade experimental .

\begin{tabular}{c|c|c|c|}
\multicolumn{1}{c}{ Coluna 1 } & \multicolumn{1}{c}{ Coluna 2 } & Coluna 3 \\
\cline { 2 - 4 } Linha 1 & $\begin{array}{c}\text { Tratamento 1 (A) } \\
\left(\mathrm{n}_{111}=1\right)\end{array}$ & $\begin{array}{c}\text { Tratamento 2 (B) } \\
\left(\mathrm{n}_{122}=2\right)\end{array}$ & $\begin{array}{c}\text { Tratamento 3 (C) } \\
\left(\mathrm{n}_{133}=4\right)\end{array}$ \\
\cline { 2 - 4 } Linha 2 & $\begin{array}{c}\text { Tratamento 3 (C) } \\
\left(\mathrm{n}_{213}=5\right)\end{array}$ & $\begin{array}{c}\text { Tratamento 1 (A) } \\
\left(\mathrm{n}_{221}=3\right)\end{array}$ & $\begin{array}{c}\text { Tratamento 2 (B) } \\
\left(\mathrm{n}_{232}=3\right)\end{array}$ \\
\cline { 2 - 4 } Linha 3 & Tratamento 2 (B) & Tratamento 3 (C) & Tratamento 1 (A) \\
$\left(\mathrm{n}_{312}=2\right)$ & $\left(\mathrm{n}_{323}=6\right)$ & $\left(\mathrm{n}_{331}=1\right)$ \\
\hline
\end{tabular}

Deve-se salientar que, para facilitar a interpretação, adotou-se um quadrado latino sistemático, mas naturalmente, na prática os tratamentos são casualizados tanto nas linhas como nas colunas, de tal forma que cada tratamento ocorra somente uma vez em cada linha e em cada coluna.

\subsection{Modelo Matemático}

O modelo matemático adotado para o ensaio em quadrado latino com $n_{\mathrm{ijk}}$ observações por unidade experimental foi o modelo superparametrizado, denotado por IEMMA (1993) por modelo S , dado por: 


$$
y_{i j k r}=\mu+\alpha_{i}+\beta_{j}+t_{k}+e_{i j k r}
$$

$$
\begin{gathered}
\operatorname{com} \mathrm{i}=1,2, \ldots, \mathrm{I} ; \mathrm{j}=1,2, \ldots, \mathrm{J} ; \mathrm{k}=1,2, \ldots, \mathrm{K} ; \mathrm{r}=1,2, \ldots, \mathrm{n}_{\mathrm{ijk}} ; \\
\mathrm{I}=\mathrm{J}=\mathrm{K} ;
\end{gathered}
$$

onde:

$y_{\mathrm{ijkr}}$ é o r-ésimo valor observado na unidade experimental referente à i-ésima linha, na j-ésima coluna e no k-ésimo tratamento ; $\mu$ é uma constante inerente a todas as observações ;

$\alpha_{i}$ é o efeito da i-ésima linha ;

$\beta_{\mathrm{j}}$ é o efeito da j-ésima coluna ;

$\mathrm{t}_{\mathrm{k}}$ é o efeito do k-ésimo tratamento ;

$\mathrm{e}_{\mathrm{ijkr}}$ é o erro aleatório atribuído à observação $\mathrm{y}_{\mathrm{ijkr}}$ tal que

$$
\mathrm{e}_{\mathrm{ijkr}} \cap \mathrm{NID}\left(0, \sigma_{\mathrm{e}}^{2}\right)
$$

Segundo SEARLE (1987), um modelo é dito superparametrizado se apresenta explicitamente um parâmetro para cada efeito dos fatores envolvidos. 
Admitindo o modelo linear de Gauss-Markov citado em $(\pi .1)$, agora na forma matricial, ou seja :

$$
\mathrm{y}=\mathrm{X} \theta+\mathrm{e}
$$

onde:

y é o vetor de realizações de variáveis aleatórias, de dimensões (n...) $\times 1$;

$\mathrm{X}$ é a matriz do delineamento, de dimensões (n...) $\times(\mathrm{I}+\mathrm{J}+\mathrm{K}+1)$;

$\theta$ é o vetor dos parâmetros desconhecidos, de dimensões $(\mathrm{I}+\mathrm{J}+\mathrm{K}+1)$ $\mathrm{x} 1$;

e é o vetor de variáveis aleatórias não observáveis, de dimensões $(\mathrm{n} . .) \times 1$, tal que $\mathrm{e} \cap \operatorname{NID}\left(\phi, I \sigma_{\mathrm{e}}^{2}\right)$;

e tomando-se o esquema do exemplo apresentado no quadro 12 , pode-se escrever: 
37.

$\left[\begin{array}{l}y_{1111} \\ y_{1221} \\ y_{1222} \\ y_{1331} \\ y_{1332} \\ y_{1333} \\ y_{1334} \\ y_{2131} \\ y_{2132} \\ y_{2133} \\ y_{2134} \\ y_{2135} \\ y_{2211} \\ y_{2212} \\ y_{2213} \\ y_{2321} \\ y_{2322} \\ y_{2323} \\ y_{3121} \\ y_{3122} \\ y_{3231} \\ y_{3232} \\ y_{3233} \\ y_{3234} \\ y_{3235} \\ y_{3236} \\ y_{3311}\end{array}\right]=\left[\begin{array}{llllllllll}1 & 1 & 0 & 0 & 1 & 0 & 0 & 1 & 0 & 0 \\ 1 & 1 & 0 & 0 & 0 & 1 & 0 & 0 & 1 & 0 \\ 1 & 1 & 0 & 0 & 0 & 1 & 0 & 0 & 1 & 0 \\ 1 & 1 & 0 & 0 & 0 & 0 & 1 & 0 & 0 & 1 \\ 1 & 1 & 0 & 0 & 0 & 0 & 1 & 0 & 0 & 1 \\ 1 & 1 & 0 & 0 & 0 & 0 & 1 & 0 & 0 & 1 \\ 1 & 1 & 0 & 0 & 0 & 0 & 1 & 0 & 0 & 1 \\ 1 & 0 & 1 & 0 & 1 & 0 & 0 & 0 & 0 & 1 \\ 1 & 0 & 1 & 0 & 1 & 0 & 0 & 0 & 0 & 1 \\ 1 & 0 & 1 & 0 & 1 & 0 & 0 & 0 & 0 & 1 \\ 1 & 0 & 1 & 0 & 1 & 0 & 0 & 0 & 0 & 1 \\ 1 & 0 & 1 & 0 & 1 & 0 & 0 & 0 & 0 & 1 \\ 1 & 0 & 1 & 0 & 0 & 1 & 0 & 1 & 0 & 0 \\ 1 & 0 & 1 & 0 & 0 & 1 & 0 & 1 & 0 & 0 \\ 1 & 0 & 1 & 0 & 0 & 1 & 0 & 1 & 0 & 0 \\ 1 & 0 & 1 & 0 & 0 & 0 & 1 & 0 & 1 & 0 \\ 1 & 0 & 1 & 0 & 0 & 0 & 1 & 0 & 1 & 0 \\ 1 & 0 & 1 & 0 & 0 & 0 & 1 & 0 & 1 & 0 \\ 1 & 0 & 0 & 1 & 1 & 0 & 0 & 0 & 1 & 0 \\ 1 & 0 & 0 & 1 & 1 & 0 & 0 & 0 & 1 & 0 \\ 1 & 0 & 0 & 1 & 0 & 1 & 0 & 0 & 0 & 1 \\ 1 & 0 & 0 & 1 & 0 & 1 & 0 & 0 & 0 & 1 \\ 1 & 0 & 0 & 1 & 0 & 1 & 0 & 0 & 0 & 1 \\ 1 & 0 & 0 & 1 & 0 & 1 & 0 & 0 & 0 & 1 \\ 1 & 0 & 0 & 1 & 0 & 1 & 0 & 0 & 0 & 1 \\ 1 & 0 & 0 & 1 & 0 & 1 & 0 & 0 & 0 & 1 \\ 1 & 0 & 0 & 1 & 0 & 0 & 1 & 1 & 0 & 0\end{array}\right]\left[\begin{array}{l}\mu \\ \alpha_{1} \\ \alpha_{2} \\ \alpha_{3} \\ \beta_{1} \\ \beta_{2} \\ \beta_{3} \\ t_{1} \\ t_{2} \\ t_{3}\end{array}\right]+\left[\begin{array}{l}e_{1111} \\ e_{1221} \\ e_{1222} \\ e_{1331} \\ e_{1332} \\ e_{1333} \\ e_{1334} \\ e_{2131} \\ e_{2132} \\ e_{2133} \\ e_{2134} \\ e_{2135} \\ e_{2211} \\ e_{2212} \\ e_{2213} \\ e_{2321} \\ e_{2322} \\ e_{2323} \\ e_{3121} \\ e_{3122} \\ e_{3231} \\ e_{3232} \\ e_{3233} \\ e_{3234} \\ e_{3235} \\ e_{3236} \\ e_{3311}\end{array}\right]$




\subsection{Equações Normais}

Sabendo que o vetor e é de variáveis aleatórias não observáveis, sua estimação depende das características do modelo e da solução do sistema de equações lineares $\mathrm{y}=\mathrm{X} \theta$ que é inconsistente. Uma solução pode ser obtida através do sistema de equações normais

$$
X^{\prime} X \theta=X^{\prime} y
$$

sempre consistente. Qualquer solução $\theta^{0}$, exata, do sistema de equações normais $\mathrm{X}^{\prime} \mathrm{X} \theta=\mathrm{X}^{\prime} \mathrm{y}$ é solução aproximada de mínimos quadrados do sistema inconsistente $y=X \theta$. Desta forma, para qualquer solução $\theta^{0}$ do sistema de equações normais tem-se o vetor $\hat{\mathrm{y}}=\mathrm{X} \theta^{0}$ invariante, o qual é a aproximação de mínimos quadrados para o vetor das observações (IEMMA \& PALM, 1992).

Se a matriz $\mathrm{X}$ tem posto coluna incompleto, então $\mathrm{X}^{\prime} \mathrm{X}$ não é não-singular e o sistema de equações normais é indeterminado. As soluções exatas deste sistema podem ser obtidas, dentre outras, por:

$$
\theta^{0}=\left(X^{\prime} X\right)^{G} X^{\prime} y
$$

onde $\left(X^{\prime} X\right)^{G}$ é uma matriz inversa generalizada qualquer de $X^{\prime} X$.

Essas soluções são soluções aproximadas de mínimos quadrados de $y=X \theta$ inconsistente. 
Como, no modelo em questão, $\mathrm{X}$ tem posto coluna incompleto os componentes do vetor $\theta$ não são estimáveis isoladamente, mas, segundo Rao (1945), citado por IEMMA (1993), "uma função linear paramétrica $\lambda^{\prime} \theta$ é estimável, no modelo linear de Gauss-Markov, se e somente se $\lambda \in \mathrm{C}\left(\mathrm{X}^{\prime}\right)$ ". Em termos práticos, tem-se $\mathrm{E}(\mathrm{y})=\mathrm{X} \theta$, então $\mathrm{X} \theta$ é estimável . Além disso, combinações lineares de funções estimáveis são também estimáveis, a menos de desconexões.

Acrescente-se ainda que, pelo teorema de Gauss-Markov, se $\lambda^{\prime} \theta$ é estimável, então seu BLUE ("Best Linear Unbiased Estimator") é único e dado por $\lambda^{\prime} \theta=\lambda^{\prime} \theta^{0}$, onde $\theta^{0}$ é qualquer solução do sistema de equações normais.

Para o caso de quadrado latino com $n_{\mathrm{ijk}}$ observações por casela, tomando o exemplo apresentado no quadro 12 , pode-se considerar a matriz X , desmembrada da seguinte forma:

$$
\mathrm{X}=\left[\begin{array}{lllllll}
\mathrm{X}_{1} & \vdots & \mathrm{X}_{2} & \vdots & \mathrm{X}_{3} & \vdots & \mathrm{X}_{4}
\end{array}\right]
$$

onde:

$\mathrm{X}_{1}, \mathrm{X}_{2}, \mathrm{X}_{3}$ e $\mathrm{X}_{4}$ são matrizes constituídas de zeros e uns tais que:

$\mathrm{X}_{1}$ é o vetor que envolve a constante inerente a todas as observações de dimensões $(\mathrm{n} . .) \times$.1 ;

$\mathrm{X}_{2}$ é a matriz associada aos efeitos de linhas, de dimensões (n...) x I ;

$\mathrm{X}_{3}$ é a matriz associada aos efeitos de colunas, de dimensões (n...) $\mathrm{x} J$; 
$\mathrm{X}_{4}$ é a matriz associada aos efeitos de tratamentos, de dimensões (n...) $\times \mathrm{K}$.

É imediato verificar que, em ( $\pi .3$ ), a matriz $X_{1}$ é constituída da $1^{\text {a }}$ coluna de $X ; X_{2}$ das colunas 2,3 e $4 ; X_{3}$ das colunas 5,6 e $7 ; X_{4}$ das colunas 8,9 e 10 .

Assim, X'X simétrica é dada por:

$$
X^{\prime} X=\left[\begin{array}{llll}
X_{1}^{\prime} X_{1} & X_{1}^{\prime} X_{2} & X_{1}^{\prime} X_{3} & X_{1}^{\prime} X_{4} \\
X_{2}^{\prime} X_{1} & X_{2}^{\prime} X_{2} & X_{2}^{\prime} X_{3} & X_{2}^{\prime} X_{4} \\
X_{3}^{\prime} X_{1} & X_{3}^{\prime} X_{2} & X_{3}^{\prime} X_{3} & X_{3}^{\prime} X_{4} \\
X_{4}^{\prime} X_{1} & X_{4}^{\prime} X_{2} & X_{4}^{\prime} X_{3} & X_{4}^{\prime} X_{4}
\end{array}\right]
$$

onde as submatrizes têm a seguinte composição:

$\mathrm{X}_{1}{ }^{\prime} \mathrm{X}_{1}=\mathrm{n} \ldots$ é o número total de observações ;

$X_{1}^{\prime} X_{2}=\left[n_{1 . .}, n_{2 . .}, \ldots, n_{1 .}\right]$ é o vetor associado ao número de observações por linha ;

$\mathrm{X}_{1}^{\prime} \mathrm{X}_{3}=\left[\mathrm{n}_{1,}, \mathrm{n}_{2,}, \ldots, \mathrm{n}_{\mathrm{J}}\right]$ é o vetor associado ao número de observações por coluna ;

$\mathrm{X}_{1}{ }^{\prime} \mathrm{X}_{4}=\left[\mathrm{n}_{. .1}, \mathrm{n}_{. .2}, \ldots, \mathrm{n}_{. \mathrm{K}}\right]$ é o vetor associado ao número de observações por tratamento ;

$\mathrm{X}_{2}{ }^{\prime} \mathrm{X}_{2}=\operatorname{diag}\left[\mathrm{n}_{1 . .}, \mathrm{n}_{2 . .}, \ldots, \mathrm{n}_{\mathrm{L} .}\right]$ é a matriz diagonal do número de observações por linha, de dimensões I x I ; 
$\mathrm{X}_{2}{ }^{\prime} \mathrm{X}_{3}=_{\mathrm{I}}\left[\mathrm{n}_{\mathrm{ijk}}\right]_{I}$ é a matriz de incidência dos tratamentos nas linhas e nas colunas, sendo $n_{i j k}$ o número de observações que cada combinação ijk fornece ;

$\mathrm{X}_{2}^{\prime} \mathrm{X}_{4}$ e $\mathrm{X}_{3}{ }^{\prime} \mathrm{X}_{4}$ são matrizes de incidência dos tratamentos nas linhas e nas colunas, sendo semelhantes à $X_{2}{ }^{\prime} X_{3}=_{1}\left[n_{i j}\right]_{I}$, com os índices reorganizados;

$\mathrm{X}_{3}{ }^{\prime} \mathrm{X}_{3}=\operatorname{diag}\left[\mathrm{n}_{1 .}, \mathrm{n}_{.2}, \ldots, \mathrm{n}_{\mathrm{J}}\right]$ é a matriz diagonal do número de observações por coluna, de dimensões $\mathrm{J} \times \mathrm{J}$;

$\mathrm{X}_{4}{ }^{\prime} \mathrm{X}_{4}=\operatorname{diag}\left[\mathrm{n}_{.1}, \mathrm{n}_{. .2}, \ldots, \mathrm{n}_{. \mathrm{K}}\right]$ é a matriz diagonal do número de observações por tratamento, de dimensões $\mathrm{K}$ x K .

Desta forma, obtém-se o seguinte sistema de equações normais $\mathrm{X}^{\prime} \mathrm{X} \theta=\mathrm{X}^{\prime} \mathrm{y}$;

$$
\left[\begin{array}{llll}
X_{1}^{\prime} X_{1} & X_{1}^{\prime} X_{2} & X_{1}^{\prime} X_{3} & X_{1} X_{4} \\
X_{2}^{\prime} X_{1} & X_{2}^{\prime} X_{2} & X_{2}^{\prime} X_{3} & X_{2}^{\prime} X_{4} \\
X_{3}^{\prime} X_{1} & X_{3}^{\prime} X_{2} & X_{3}^{\prime} X_{3} & X_{3}^{\prime} X_{4} \\
X_{4}^{\prime} X_{1} & X_{4}^{\prime} X_{2} & X_{4}^{\prime} X_{3} & X_{4}^{\prime} X_{4}
\end{array}\right]\left[\begin{array}{c}
\mu \\
\alpha \\
\beta \\
t
\end{array}\right]=\left[\begin{array}{c}
X_{1}^{\prime} y \\
X_{2}^{\prime} y \\
X_{3}^{\prime} y \\
X_{4}^{\prime} y
\end{array}\right]=\left[\begin{array}{c}
G \\
A \\
B \\
T
\end{array}\right]
$$

onde: 
$\alpha=\left[\begin{array}{c}\alpha_{1} \\ \alpha_{2} \\ \cdots \\ \alpha_{I}\end{array}\right]$ é o vetor dos efeitos de linhas ;

$\beta=\left[\begin{array}{c}\beta_{1} \\ \beta_{2} \\ \ldots \\ \beta_{\mathrm{J}}\end{array}\right]$ é o vetor dos efeitos de colunas ;

$t=\left[\begin{array}{c}t_{1} \\ t_{2} \\ \cdots \\ t_{K}\end{array}\right]$ é o vetor dos efeitos de tratamentos;

e $\quad X^{\prime} y=\left[\begin{array}{l}X_{1}^{\prime} y \\ X_{2}^{\prime} y \\ X_{3}^{\prime} y \\ X_{4}^{\prime} y\end{array}\right]=\left[\begin{array}{c}G \\ A \\ B \\ T\end{array}\right]$

onde:

G é o total de observações do experimento; 
$A=\left[\begin{array}{c}A_{1} \\ A_{2} \\ \ldots \\ A_{I}\end{array}\right]$ é o vetor dos totais observados para as linhas ;

$B=\left[\begin{array}{c}B_{1} \\ B_{2} \\ \ldots \\ B_{J}\end{array}\right]$ é o vetor dos totais observados para as colunas ;

$\mathrm{T}=\left[\begin{array}{c}\mathrm{T}_{1} \\ \mathrm{~T}_{2} \\ \ldots \\ \mathrm{T}_{\mathrm{K}}\end{array}\right]$ é o vetor dos totais observados para os tratamentos

\subsection{Análise da Variância}

Ainda através da teoria de modelos lineares tem-se que $\hat{\mathrm{y}}=\mathrm{X} \theta^{0}$ é a aproximação de mínimos quadrados para o vetor de observações y, invariante para qualquer solução $\theta^{0}$ do sistema de equações normais, ou ainda:

$$
\hat{y}=X\left(X^{\prime} X\right)^{G} X^{\prime} y=P y
$$


onde, segundo IEMMA \& PALM (1992), $\mathrm{P}=\mathrm{X}\left(\mathrm{X}^{\prime} \mathrm{X}\right)^{\mathrm{G}} \mathrm{X}^{\prime}=\mathrm{XX}^{+}=\mathrm{XX}^{1}$ é o projetor ortogonal de y sobre o espaço gerado pelas colunas de X e ( I - P) é o projetor ortogonal de y sobre o complemento ortogonal do espaço coluna de $\mathrm{X}, \mathrm{C}^{\perp}(\mathrm{X})$.

Assim, tem-se a decomposição clássica da análise da variância :

$$
\|y\|^{2}=\|\hat{y}\|^{2}+\|\hat{\mathrm{e}}\|^{2}
$$

onde:

$$
\begin{aligned}
& \|y\|^{2}=y^{\prime} y=\sum_{i \mathrm{j} k r} y_{\mathrm{ijkr}}^{2}=\text { S.Q.Total ; } \\
& \|\hat{y}\|^{2}=\hat{y}^{\prime} \hat{y}=\sum_{\mathrm{ijks}} \hat{y}_{\mathrm{ijkr}}^{2}=y^{\prime} \mathrm{Py}=\theta^{0} \mathrm{X}^{\prime} \mathrm{y}=\mathrm{S} \text {.Q.Parâmetros }=\mathrm{R}(\mu, \alpha, \beta, \mathrm{t}) ;(\pi .10) \\
& \|\hat{e}\|^{2}=\hat{e}^{\prime} \hat{e}=\sum_{\mathrm{ijkr}} \hat{e}_{\mathrm{j} j \mathrm{kr}}^{2}=y^{\prime} y-\theta^{0} X^{\prime} y=\text { S.Q.Resíduo . }
\end{aligned}
$$

Segundo IEMMA (1995a), dentre outros, as somas de quadrados associadas ao modelo podem ser convenientemente apresentadas através da notação $\mathrm{R}($ ), principalmente no caso do modelo superparametrizado aqui utilizado. Um inconveniente desta notação é não indicar a hipótese que está sendo testada.

A obtenção da soma de quadrados de hipóteses de interesse é uma alternativa muito útil. Assim, para a hipótese : 


$$
\mathrm{H}_{0}: \mathrm{B}^{\prime} \theta=\phi
$$

onde $\mathrm{B}^{\prime} \theta$ é estimável e $\mathrm{B}^{\prime}$ tem posto linha completo, tem-se:

$$
\text { S.Q. } H_{0}=\left(B^{\prime} \theta^{0}\right)^{\prime}\left[B^{\prime}\left(X^{\prime} X\right)^{G} B\right]^{-1}\left(B^{\prime} \theta^{0}\right)
$$

e verifica-se que

$$
\frac{\text { S.Q.H }}{\sigma^{2}} \cap \chi_{\{\mathrm{r}[X\} ; 8\}}^{2}
$$

onde $\delta=\frac{1}{2 \sigma^{2}} \theta^{\prime} \mathrm{X}^{\prime} \mathrm{P}^{*} \mathrm{X} \theta$ é o parâmetro de não centralidade e $\mathrm{P}^{*}$ é o projetor ortogonal de $\mathrm{y}$, tal que $\mathrm{y}^{\prime} \mathbf{P}^{*} \mathrm{y}=\mathrm{S} \cdot \mathrm{Q} \cdot \mathrm{H}_{0}$.

Salienta-se ainda que se $\mathrm{P}^{*}\left(\mathrm{I}-\mathrm{P}^{*}\right)=\phi$ então, dada a hipótese de normalidade, as formas quadráticas associadas são independentes.

Ademais, sendo $\mathrm{P}^{*}$ matriz simétrica, idempotente de posto $\mathrm{r}[\mathrm{B}]$, o quociente dos respectivos quadrados médios tem distribuição F não central, ou seja

$$
\frac{\text { Q.M.H }}{\text { Q.M.Resíduo }} \cap \mathrm{F}_{\{\mathrm{r}[\mathrm{B}], \mathrm{r}[\mathrm{I}-\mathrm{P}], \delta\}}
$$

e

$$
\mathrm{E}\left[\mathrm{S} \cdot \mathrm{Q} \cdot \mathrm{H}_{0}\right]=\mathrm{E}\left[\mathrm{y}^{\prime} \mathrm{P}^{*} \mathrm{y}\right]=\operatorname{Tr}\left[\mathrm{P}^{*}\right] \sigma^{2}+\theta^{\prime} \mathrm{X}^{\prime} \mathrm{P}^{*} \mathrm{X} \theta
$$




\subsection{Notação R( )}

Admitindo o modelo dado anteriormente, no item (3.2.), ou seja:

$$
y=X \theta+e
$$

a notação $\mathrm{R}($ ) é, conforme ( $\pi .10)$, dada por:

$$
\text { S.Q. }(\mu, \alpha, \beta, t)=R(\theta)=\theta^{0} X^{\prime} y \text {; }
$$

onde $\theta^{0}$ é uma solução para $\mathrm{X}^{\prime} \mathrm{X} \theta=\mathrm{X}^{\prime} \mathrm{Y}$.

Tomando X particionada como em $(\pi .6)$, tem-se

$$
\mathrm{X}=\left[\begin{array}{lllllll}
\mathrm{X}_{1} & \vdots & \mathrm{X}_{2} & \vdots & \mathrm{X}_{3} & \vdots & \mathrm{X}_{4}
\end{array}\right],
$$

e conseqüentemente 
$\theta=\left[\begin{array}{c}\theta_{1} \\ -- \\ \theta_{2} \\ -- \\ \theta_{3} \\ -- \\ \theta_{4}\end{array}\right]=\left[\begin{array}{c}\mu \\ --- \\ \alpha_{1} \\ \alpha_{2} \\ \vdots \\ \alpha_{\mathrm{I}} \\ --- \\ \beta_{1} \\ \beta_{2} \\ \vdots \\ \beta_{\mathrm{J}} \\ --- \\ \mathrm{t}_{1} \\ \mathrm{t}_{2} \\ \vdots \\ \mathrm{t}_{\mathrm{K}}\end{array}\right]$

Utilizando-se a parametrização sucessiva desde o modelo mais simples, que contém apenas a constante, até o modelo dado em 3.2. , podem-se obter as somas de quadrados de interesse, como a seguir : 
3.5.1. Redução na soma de quadrados total devida ao ajuste da constante $\mu$.

Tomando o modelo

$$
y=X_{1} \theta_{1}+e_{1}
$$

caracterizado por $\mathrm{y}_{\mathrm{j} \mathrm{k} k \mathrm{r}}=\mu+\mathrm{e}_{\mathrm{ijkr}}^{(1)}$, vem, segundo ( $\left.\pi .6\right)$

$$
\mathrm{X}_{1}{ }^{\prime} \mathrm{X}_{\mathrm{l}} \theta_{1}=\mathrm{X}_{1}{ }^{\prime} \mathrm{y}=\mathrm{n}_{\ldots} \boldsymbol{\mu}=\mathrm{G}
$$

e então $\theta_{1}^{0}=\mu^{0}=\frac{G}{n}$.

Desse modo, através de $(\pi .10)$ ou $(\pi .16)$, tem-se :

$$
R(\mu)=C=\theta_{1}^{0} X_{1}{ }^{\prime} y=\frac{G^{2}}{n_{\ldots .}}=n \bar{y}^{2}
$$

3.5.2. Redução na soma de quadrados total devida ao ajuste de linhas após $o$ ajuste da constante

Admitindo o modelo

$$
y=X_{12} \theta_{12}+e_{2},
$$


caracterizado por $\mathrm{y}_{\mathrm{ijkr}}=\mu+\alpha_{\mathrm{i}}+\mathrm{e}_{\mathrm{ijkr}}^{(2)}$, vem, segundo $(\pi .6)$

$$
\mathrm{X}_{12}^{\prime} \mathrm{X}_{12} \theta_{12}=\mathrm{X}_{12}^{\prime} \mathrm{y}
$$

Desse modo, tem-se, através de $(\pi .10)$ ou $(\pi .16)$ :

$$
\mathrm{R}(\mu, \alpha)=\theta_{12}^{0} \mathrm{X}_{12}^{\prime} \mathrm{y}=\mathrm{S} . \mathrm{Q} .(\mu, \alpha)
$$

onde

$$
X_{12}=\left[\begin{array}{lll}
X_{1} & 1 & X_{2}
\end{array}\right] \quad \text { e } \quad \theta_{12}=\left[\begin{array}{c}
\theta_{1} \\
-- \\
\theta_{2}
\end{array}\right]
$$

Portanto, pode-se obter

$$
\mathrm{R}(\alpha \mid \mu)=\mathrm{R}(\mu, \alpha)-\mathrm{R}(\mu)=\sum_{\mathrm{i}} \frac{\mathrm{A}_{\mathrm{i}}^{2}}{\mathrm{n}_{\mathrm{i} . .}}-\mathrm{C}
$$

3.5.3. Redução na soma de quadrados total devida ao ajuste de colunas após o ajuste da constante

De modo análogo, admitindo o modelo

$$
y=X_{13} \theta_{13}+e_{3}
$$


caracterizado por $\mathrm{y}_{\mathrm{ijkr}}=\mu+\beta_{\mathrm{i}}+\mathrm{e}_{\mathrm{ijkr}}^{(3)}$, vem, segundo $(\pi .6)$

$$
X_{13}^{\prime} X_{13} \theta_{13}=X_{13}^{\prime} y
$$

Desse modo, tem-se, através de $(\pi .10)$ ou $(\pi .16)$ :

$$
R(\mu, \beta)=\theta_{13}^{0} X_{13}^{\prime} y=S . Q .(\mu, \beta)
$$

onde

$$
X_{13}=\left[\begin{array}{l:ll}
X_{1} & X_{3}
\end{array}\right] \quad \text { e } \quad \theta_{13}=\left[\begin{array}{c}
\theta_{1} \\
-- \\
\theta_{3}
\end{array}\right]
$$

Portanto, pode-se obter

$$
R(\beta \mid \mu)=R(\mu, \beta)-R(\mu)=\sum_{j} \frac{B_{j}^{2}}{n_{. j}}-C
$$

3.5.4. Redução na soma de quadrados total devida ao ajuste de tratamentos após o ajuste da constante

Tomando, de modo análogo aos anteriores, o modelo

$$
\mathrm{y}=\mathrm{X}_{14} \theta_{14}+\mathrm{e}_{4}
$$


caracterizado por $\mathrm{y}_{\mathrm{ijkr}}=\mu+\alpha_{\mathrm{i}}+\mathrm{e}_{\mathrm{ijkr}}^{(4)}$, vem, segundo $(\pi .6)$

$$
\mathrm{X}_{14}^{\prime} \mathrm{X}_{14} \theta_{14}=\mathrm{X}_{14}^{\prime} \mathrm{y}
$$

Então, tem-se, através de $(\pi .10)$ ou $(\pi .16)$ :

$$
R(\mu, t)=\theta_{14}^{0} X_{14}^{\prime} y=S . Q \cdot(\mu, t)
$$

onde

$$
X_{14}=\left[\begin{array}{lll}
X_{1} & 1 & X_{4}
\end{array}\right] \quad \text { e } \quad \theta_{14}=\left[\begin{array}{c}
\theta_{1} \\
-- \\
\theta_{4}
\end{array}\right]
$$

Portanto, pode-se obter

$$
R(t \mid \mu)=R(\mu, t)-R(\mu)=\sum_{k} \frac{T_{k}^{2}}{n_{. . k}}-C
$$

3.5.5. Redução na soma de quadrados total devida ao ajuste de linhas após o ajuste de colunas e da constante

Tomando, de forma análoga, o modelo

$$
y=X_{123} \theta_{123}+e_{5}
$$


caracterizado por $\mathrm{y}_{\mathrm{ijkr}}=\mu+\alpha_{\mathrm{i}}+\beta_{\mathrm{j}}+\mathrm{e}_{\mathrm{ij \textrm {kr }}}^{(5)}$, tem-se :

$$
\begin{gathered}
\mathrm{X}_{123}{ }^{\prime} \mathrm{X}_{123} \theta_{123}=\mathrm{X}_{123}{ }^{\prime} \mathrm{y} \\
\text { Então, } \mathrm{R}(\mu, \alpha, \beta)=\theta_{123}^{0}{ }^{\prime} \mathrm{X}_{123}{ }^{\prime} \mathrm{y}=\mathrm{S} . \mathrm{Q} .(\mu, \alpha, \beta),
\end{gathered}
$$

onde :

$$
X_{123}=\left[\begin{array}{lllll}
X_{1} & \vdots & X_{2} & \vdots & X_{3}
\end{array}\right] \quad \text { e } \quad \theta_{123}=\left[\begin{array}{c}
\theta_{1} \\
-- \\
\theta_{2} \\
\hdashline \\
\theta_{3}
\end{array}\right]
$$

e, portanto, $\mathrm{R}(\alpha \mid \mu, \beta)=\mathrm{R}(\mu, \alpha, \beta)-\mathrm{R}(\mu, \beta)$.

3.5.6. Redução na soma de quadrados total devida ao ajuste de linhas após o ajuste de tratamentos e da constante

Analogamente ao item anterior, tomando o modelo

$$
y=X_{124} \theta_{124}+e_{6}
$$

caracterizado por $y_{i j k r}=\mu+\alpha_{i}+t_{k}+e_{i j k r}^{(6)}$, tem-se : 


$$
\mathrm{X}_{124}{ }^{\prime} \mathrm{X}_{124} \theta_{124}=\mathrm{X}_{124}{ }^{\prime} \mathrm{y}
$$

$$
\text { Então, } R(\mu, \alpha, t)=\theta_{124}^{0}{ }^{\prime} X_{124}{ }^{\prime} y=S . Q .(\mu, \alpha, t) \text {, }
$$

onde :

$$
X_{124}=\left[\begin{array}{lll:l}
X_{1} & X_{2} & X_{4}
\end{array}\right] \text { e } \theta_{124}=\left[\begin{array}{c}
\theta_{1} \\
- \\
\theta_{2} \\
\hdashline \\
\theta_{4}
\end{array}\right]
$$

e portanto $R(\alpha \mid \mu, t)=R(\mu, \alpha, t)-R(\mu, t)$.

3.5.7. Redução na soma de quadrados total devida ao ajuste de colunas após o ajuste de linhas e da constante

De forma análoga, tomando os resultados dos itens (3.5.2) e (3.5.5), tem-se :

$$
\mathrm{R}(\beta \mid \mu, \alpha)=\mathrm{R}(\mu, \alpha, \beta)-\mathrm{R}(\mu, \alpha) .
$$


3.5.8. Redução na soma de quadrados total devida ao ajuste de colunas após 0 ajuste de tratamentos e da constante

Analogamente aos itens anteriores, tomando o modelo

$$
\mathrm{y}=\mathrm{X}_{134} \theta_{134}+\mathrm{e}_{7}
$$

caracterizado por $\mathrm{y}_{\mathrm{ijkr}}=\mu+\beta_{\mathrm{k}}+\mathrm{t}_{\mathrm{k}}+\mathrm{e}_{\mathrm{ijkr}}^{(7)}$, tem-se :

$$
\mathrm{X}_{134}{ }^{\prime} \mathrm{X}_{134} \theta_{134}=\mathrm{X}_{134}{ }^{\prime} \mathrm{y}
$$

Desse modo, $R(\mu, \beta, t)=\theta_{134}^{0}{ }^{\prime} X_{134}{ }^{\prime} y=S . Q .(\mu, \beta, t)$, onde :

$$
X_{134}=\left[\begin{array}{l:ll:l}
X_{1} & X_{3} & X_{4}
\end{array}\right] \text { e } \quad \theta_{134}=\left[\begin{array}{c}
\theta_{1} \\
- \\
\theta_{3} \\
- \\
\theta_{4}
\end{array}\right]
$$

e ainda $R(\beta \mid \mu, t)=R(\mu, \beta, t)-R(\mu, t)$. 
3.5.9. Redução na soma de quadrados total devida ao ajuste de tratamentos após o ajuste de linhas e da constante

De modo análogo, tomando os resultados dos itens (3.5.2) e (3.5.6), tem-se :

$$
\mathrm{R}(\mathrm{t} \mid \mu, \alpha)=\mathrm{R}(\mu, \alpha, \mathrm{t})-\mathrm{R}(\mu, \alpha)
$$

3.5.10. Redução na soma de quadrados total devida ao ajuste de tratamentos após o ajuste de colunas e da constante Tomando, de modo análogo, os resultados dos itens (3.5.3) e (3.5.8), tem-se :

$$
\mathrm{R}(\mathrm{t} \mid \mu, \boldsymbol{\beta})=\mathrm{R}(\boldsymbol{\mu}, \boldsymbol{\beta}, \mathrm{t})-\mathrm{R}(\boldsymbol{\mu}, \boldsymbol{\beta}) .
$$

3.5.11. Redução na soma de quadrados total devida ao ajuste de linhas após o ajuste de colunas, de tratamentos e da constante

Tomando a S.Q.Parâmetros $=\mathrm{R}(\mu, \alpha, \beta, t)$, conforme ( $\pi .10)$ e os resultados do item (3.5.8), tem-se :

$$
\mathrm{R}(\alpha \mid \mu, \beta, \mathrm{t})=\mathrm{R}(\mu, \alpha, \beta, \mathrm{t})-\mathrm{R}(\mu, \beta, \mathrm{t}) .
$$


3.5.12. Redução na soma de quadrados total devida ao ajuste de colunas após o ajuste de linhas, de tratamentos e da constante

De forma análoga aos itens anteriores, tem-se :

$$
\mathrm{R}(\beta \mid \mu, \alpha, \mathrm{t})=\mathrm{R}(\mu, \alpha, \beta, \mathrm{t})-\mathrm{R}(\mu, \alpha, \mathrm{t})
$$

3.5.13. Redução na soma de quadrados total devida ao ajuste de tratamentos após o ajuste de linhas, de colunas e da constante

Nesse caso, de forma análoga às anteriores, tem-se :

$$
\mathrm{R}(\mathrm{t} \mid \mu, \alpha, \beta)=\mathrm{R}(\mu, \alpha, \beta, \mathrm{t})-\mathrm{R}(\mu, \alpha, \beta)
$$

\subsection{Tipos de Funções Estimáveis}

Segundo IEMMA (1995b), que cita o conceito de Rao (1945), se a matriz $X$ tem posto coluna incompleto, então $\theta$ não tem estimador não viesado; nestas condições $E\left(\theta^{0}\right)=\left(X^{\prime} X\right)^{G} X^{\prime} X \theta=H \theta$, onde H $\theta$ é estimável. 
Dessa forma, o PROC GLM do SAS apresenta uma base para se estudar estimabilidade. Nesse procedimento, tem-se $\mathrm{L}=\left(\mathrm{X}^{\prime} \mathrm{X}\right)^{\mathrm{G} 2} \mathrm{X}^{\prime} \mathrm{X}$, analogamente à matriz $\mathrm{H}$, com diferença de que $\mathrm{G} 2$ fornece um único L . A obtenção da matriz G2 não é objetivo deste, sendo apresentada de forma detalhada em alguns textos de modelos lineares, como MONDARDO (1994).

Conforme pode ser visto no exemplo ilustrativo e no apêndice, dentre outros resultados fornecidos pelo SAS / PROC GLM, têmse os coeficientes das funções estimáveis, que são de grande valia no reconhecimento das hipóteses que estão sendo testadas através das quatro somas de quadrados.

Visando aplicações na interpretação das hipóteses testadas, apresenta-se, a seguir, uma primeira idéia sobre os quatro tipos de funções estimáveis fornecidas pelo SAS / GLM. Dados os objetivos deste estudo, não são discutidos os métodos de obtenção. Tal discussão pode ser vista em MONDARDO (1994).

\subsubsection{Forma geral de funções estimáveis}

A forma geral de funções estimáveis é dada através de $L \theta$, onde $\mathrm{L}=\left(\mathrm{X}^{\prime} \mathrm{X}\right)^{\mathrm{G} 2} \mathrm{X}^{\prime} \mathrm{X}$.

Para o caso em questão, conforme o quadro 12 , tem-se a forma geral de funções estimáveis apresentada no quadro 13 . 
Quadro 13. Forma geral das funções estimáveis para o quadrado latino apresentado no quadro 12 .

\begin{tabular}{cc}
\hline Parâmetros & Coeficientes \\
\hline$\mu$ & $\mathrm{L}_{1}$ \\
$\alpha_{1}$ & $\mathrm{~L}_{2}$ \\
$\alpha_{2}$ & $\mathrm{~L}_{3}$ \\
$\alpha_{3}$ & $\mathrm{~L}_{1}-\mathrm{L}_{2}-\mathrm{L}_{3}$ \\
$\beta_{1}$ & $\mathrm{~L}_{5}$ \\
$\beta_{2}$ & $\mathrm{~L}_{6}$ \\
$\beta_{3}$ & $\mathrm{~L}_{1}-\mathrm{L}_{5}-\mathrm{L}_{6}$ \\
$\mathbf{t}_{1}$ & $\mathrm{~L}_{8}$ \\
$\mathbf{t}_{2}$ & $\mathrm{~L}_{9}$ \\
$\mathbf{t}_{3}$ & $\mathrm{~L}_{1}-\mathrm{L}_{8}-\mathrm{L}_{9}$ \\
\hline
\end{tabular}

Portanto, pode-se escrever :

$$
\begin{aligned}
\mathrm{L} \theta= & \mathrm{L}_{1} \mu+\mathrm{L}_{2} \alpha_{1}+\mathrm{L}_{3} \alpha_{2}+\left(\mathrm{L}_{1}-\mathrm{L}_{2}-\mathrm{L}_{3}\right) \alpha_{3}+ \\
& +\mathrm{L}_{5} \beta_{1}+\mathrm{L}_{6} \beta_{2}+\left(\mathrm{L}_{1}-\mathrm{L}_{5}-\mathrm{L}_{6}\right) \beta_{3}+ \\
& +\mathrm{L}_{8} \mathrm{t}_{1}+\mathrm{L}_{9} \mathrm{t}_{2}+\left(\mathrm{L}_{1}-\mathrm{L}_{8}-\mathrm{L}_{9}\right) \mathrm{t}_{3}
\end{aligned}
$$

Atribuindo-se valores aos coeficientes L's obtêm-se as formas específicas de tais funções. 
Salienta-se que $L$ representa o posto máximo da hipótese associada, sendo assim, o número de L's está relacionado com o número de graus de liberdade da soma de quadrados referente à hipótese em questão.

\subsubsection{Funções estimáveis do tipo I}

As funções estimáveis do tipo I são obtidas, seqüencialmente, fazendo-se uma partição da matriz $\mathrm{X}$, de acordo com os parâmetros envolvidos e com a ordem em que os mesmos são apresentados.

Através delas são obtidas hipóteses do tipo $\mathrm{I}$, que são hipóteses sobre médias ponderadas não ajustadas.

Para o caso em questão, apresentado no quadro 12 , têm-se as funções estimáveis dos quadros 14 a 17 , conforme a ordenação dos efeitos no modelo . 
Quadro 14. Funções estimáveis do tipo I para efeitos de tratamentos no quadrado latino apresentado no quadro 12 , adotando a ordem linha, coluna, tratamento.

\begin{tabular}{cc}
\hline Parâmetros & Coeficientes \\
\hline$\mu$ & 0 \\
$\alpha_{1}$ & 0 \\
$\alpha_{2}$ & 0 \\
$\alpha_{3}$ & 0 \\
$\beta_{1}$ & 0 \\
$\beta_{2}$ & 0 \\
$\beta_{3}$ & 0 \\
$t_{1}$ & $\mathrm{~L}_{8}$ \\
$\mathrm{t}_{2}$ & $\mathrm{~L}_{9}$ \\
$\mathrm{t}_{3}$ & $-\mathrm{L}_{8}-\mathrm{L}_{9}$ \\
\hline
\end{tabular}

Portanto, para o quadro 14 , pode-se escrever :

$$
\mathrm{L} \theta=\mathrm{L}_{8} \mathrm{t}_{1}+\mathrm{L}_{9} \mathrm{t}_{2}-\left(\mathrm{L}_{8}+\mathrm{L}_{9}\right) \mathrm{t}_{3}
$$

Assim, atribuindo-se valores a $\mathrm{L}_{8}$ e $\mathrm{L}_{9}$ se obtém a hipótese testada através da soma de quadrados tipo I que o PROC GLM do SAS fornece. 
Por exemplo, tomando os resultados do quadro $14 \mathrm{e}$ fazendo $\mathrm{L}_{8}=1$ e $\mathrm{L}_{9}=0$ e vice-versa, tem-se :

$$
H_{0}:\left\{\begin{array}{l}
t_{1}-t_{3}=0 \\
t_{2}-t_{3}=0
\end{array}\right.
$$

Deve-se observar que, para o tipo $I$, as somas de quadrados dependem da ordem de entrada dos efeitos no modelo, pois cada efeito é ajustado para todos os anteriores. 
Quadro 15. Funções estimáveis do tipo I para efeitos de tratamentos no quadrado latino apresentado no quadro 12 , adotando a ordem linha, tratamento, coluna.

\begin{tabular}{cc}
\hline Parâmetros & Coeficientes \\
\hline$\mu$ & 0 \\
$\alpha_{1}$ & 0 \\
$\alpha_{2}$ & 0 \\
$\alpha_{3}$ & 0 \\
$\mathrm{t}_{1}$ & $\mathrm{~L}_{5}$ \\
$\mathrm{t}_{2}$ & $\mathrm{~L}_{6}$ \\
$\mathrm{t}_{3}$ & $-\mathrm{L}_{5}-\mathrm{L}_{6}$ \\
$\beta_{1}$ & $-0,2098 \mathrm{~L}_{5}-0,072 \mathrm{~L}_{6}$ \\
$\beta_{2}$ & $0,291 \mathrm{~L}_{5}-0,0652 \mathrm{~L}_{6}$ \\
$\beta_{3}$ & $-0,0811 \mathrm{~L}_{5}+0,1373 \mathrm{~L}_{6}$ \\
\hline
\end{tabular}


Quadro 16. Funções estimáveis do tipo I para efeitos de tratamentos no quadrado latino apresentado no quadro 12 , adotando a ordem coluna, tratamento, linha.

\begin{tabular}{cc}
\hline Parâmetros & Coeficientes \\
\hline$\mu$ & 0 \\
$\beta_{1}$ & 0 \\
$\beta_{2}$ & 0 \\
$\beta_{3}$ & 0 \\
$\mathrm{t}_{1}$ & $\mathrm{~L}_{5}$ \\
$\mathrm{t}_{2}$ & $\mathrm{~L}_{6}$ \\
$\mathrm{t}_{3}$ & $-\mathrm{L}_{5}-\mathrm{L}_{6}$ \\
$\alpha_{1}$ & $-0,0538 \mathrm{~L}_{5}-0,0353 \mathrm{~L}_{6}$ \\
$\alpha_{2}$ & $0,3281 \mathrm{~L}_{5}+0,0925 \mathrm{~L}_{6}$ \\
$\alpha_{3}$ & $-0,2743 \mathrm{~L}_{5}-0,0573 \mathrm{~L}_{6}$ \\
\hline
\end{tabular}


Quadro 17. Funções estimáveis do tipo I para efeitos de tratamentos no quadrado latino apresentado no quadro 12 , adotando a ordem tratamento, linha, coluna.

\begin{tabular}{cc}
\hline Parâmetros & Coeficientes \\
\hline$\mu$ & 0 \\
$\mathrm{t}_{1}$ & $\mathrm{~L}_{2}$ \\
$\mathrm{t}_{2}$ & $\mathrm{~L}_{3}$ \\
$\mathrm{t}_{3}$ & $-\mathrm{L}_{2}-\mathrm{L}_{3}$ \\
$\alpha_{1}$ & $-0,0667 \mathrm{~L}_{2}+0,019 \mathrm{~L}_{3}$ \\
$\alpha_{2}$ & $0,2667 \mathrm{~L}_{2}+0,0952 \mathrm{~L}_{3}$ \\
$\alpha_{3}$ & $-0,2 \mathrm{~L}_{2}-0,1143 \mathrm{~L}_{3}$ \\
$\beta_{1}$ & $-0,1333 \mathrm{~L}_{2}-0,0476 \mathrm{~L}_{3}$ \\
$\beta_{2}$ & $0,2 \mathrm{~L}_{2}-0,1143 \mathrm{~L}_{3}$ \\
$\beta_{3}$ & $-0,0667 \mathrm{~L}_{2}+0,1619 \mathrm{~L}_{3}$ \\
\hline
\end{tabular}

\subsubsection{Funções estimáveis do tipo II}

As funções estimáveis do tipo II para o quadrado latino esquematizado no quadro 12 são, conforme a ordenação, as apresentadas nos quadros 18 a 21 . 
Nesse caso, a ordem dos parâmetros no modelo não tem implicação no valor das somas de quadrados, pois cada fator é ajustado para todos os demais.

As hipóteses do tipo II referem-se às médias ponderadas de tratamentos, ajustadas para linhas e para colunas.

Quadro 18. Funções estimáveis do tipo II para efeitos de tratamentos no quadrado latino apresentado no quadro 12, adotando a ordem linha, coluna, tratamento.

\begin{tabular}{cc}
\hline Parâmetros & Coeficientes \\
\hline$\mu$ & 0 \\
$\alpha_{1}$ & 0 \\
$\alpha_{2}$ & 0 \\
$\alpha_{3}$ & 0 \\
$\beta_{1}$ & 0 \\
$\beta_{2}$ & 0 \\
$\beta_{3}$ & 0 \\
$\mathrm{t}_{1}$ & $\mathrm{~L}_{8}$ \\
$\mathrm{t}_{2}$ & $\mathrm{~L}_{9}$ \\
$\mathrm{t}_{3}$ & $-\mathrm{L}_{8}-\mathrm{L}_{9}$ \\
\hline
\end{tabular}


Quadro 19. Funções estimáveis do tipo II para efeitos de tratamentos no quadrado latino apresentado no quadro 12 , adotando a ordem linha, tratamento, coluna.

\begin{tabular}{cc}
\hline Parâmetros & Coeficientes \\
\hline$\mu$ & 0 \\
$\alpha_{1}$ & 0 \\
$\alpha_{2}$ & 0 \\
$\alpha_{3}$ & 0 \\
$\mathrm{t}_{1}$ & $\mathrm{~L}_{5}$ \\
$\mathrm{t}_{2}$ & $\mathrm{~L}_{6}$ \\
$\mathrm{t}_{3}$ & $-\mathrm{L}_{5}-\mathrm{L}_{6}$ \\
$\beta_{1}$ & 0 \\
$\beta_{2}$ & 0 \\
$\beta_{3}$ & 0 \\
\hline
\end{tabular}


Quadro 20. Funções estimáveis do tipo II para efeitos de tratamentos no quadrado latino apresentado no quadro 12 , adotando a ordem coluna, tratamento, linha.

\begin{tabular}{cc}
\hline Parâmetros & Coeficientes \\
\hline$\mu$ & 0 \\
$\beta_{1}$ & 0 \\
$\beta_{2}$ & 0 \\
$\beta_{3}$ & 0 \\
$\mathrm{t}_{1}$ & $\mathrm{~L}_{5}$ \\
$\mathrm{t}_{2}$ & $\mathrm{~L}_{6}$ \\
$\mathrm{t}_{3}$ & $-\mathrm{L}_{5}-\mathrm{L}_{6}$ \\
$\alpha_{1}$ & 0 \\
$\alpha_{2}$ & 0 \\
$\alpha_{3}$ & 0 \\
\hline
\end{tabular}


Quadro 21. Funções estimáveis do tipo II para efeitos de tratamentos no quadrado latino apresentado no quadro 12 , adotando a ordem tratamento, linha, coluna.

\begin{tabular}{cc}
\hline Parâmetros & Coeficientes \\
\hline$\mu$ & 0 \\
$\mathrm{t}_{1}$ & $\mathrm{~L}_{2}$ \\
$\mathrm{t}_{2}$ & $\mathrm{~L}_{3}$ \\
$\mathrm{t}_{3}$ & $-\mathrm{L}_{2}-\mathrm{L}_{3}$ \\
$\alpha_{1}$ & 0 \\
$\alpha_{2}$ & 0 \\
$\alpha_{3}$ & 0 \\
$\beta_{1}$ & 0 \\
$\beta_{2}$ & 0 \\
$\beta_{3}$ & 0 \\
\hline
\end{tabular}

\subsubsection{Funções estimáveis do tipo III}

As funções estimáveis do tipo III para o caso em estudo são, segundo a ordenação, as apresentadas nos quadros 22 a 25 .

Nesse caso, as hipóteses constituídas de funções do tipo III referem-se às médias não ponderadas de tratamentos. 
Será visto em itens seguintes que, sendo o modelo sem interação, as somas de quadrados dos tipos II , III e IV são iguais.

Quadro 22. Funções estimáveis do tipo III para efeitos de tratamentos no quadrado latino apresentado no quadro 12, adotando a ordem linha, coluna, tratamento.

\begin{tabular}{cc}
\hline Parâmetros & Coeficientes \\
\hline$\mu$ & 0 \\
$\alpha_{1}$ & 0 \\
$\alpha_{2}$ & 0 \\
$\alpha_{3}$ & 0 \\
$\beta_{1}$ & 0 \\
$\beta_{2}$ & 0 \\
$\beta_{3}$ & 0 \\
$\mathrm{t}_{1}$ & $\mathrm{~L}_{8}$ \\
$\mathrm{t}_{2}$ & $\mathrm{~L}_{9}$ \\
$\mathrm{t}_{3}$ & $-\mathrm{L}_{8}-\mathrm{L}_{9}$ \\
\hline
\end{tabular}


Quadro 23. Funções estimáveis do tipo III para efeitos de tratamentos no quadrado latino apresentado no quadro 12, adotando a ordem linha, tratamento, coluna.

\begin{tabular}{cc}
\hline Parâmetros & Coeficientes \\
\hline$\mu$ & 0 \\
$\alpha_{1}$ & 0 \\
$\alpha_{2}$ & 0 \\
$\alpha_{3}$ & 0 \\
$\mathrm{t}_{1}$ & $\mathrm{~L}_{5}$ \\
$\mathrm{t}_{2}$ & $\mathrm{~L}_{6}$ \\
$\mathrm{t}_{3}$ & $-\mathrm{L}_{5}-\mathrm{L}_{6}$ \\
$\beta_{1}$ & 0 \\
$\beta_{2}$ & 0 \\
$\beta_{3}$ & 0 \\
\hline
\end{tabular}


Quadro 24. Funções estimáveis do tipo III para efeitos de tratamentos no quadrado latino apresentado no quadro 12 , adotando a ordem coluna, tratamento, linha.

\begin{tabular}{cc}
\hline Parâmetros & Coeficientes \\
\hline$\mu$ & 0 \\
$\beta_{1}$ & 0 \\
$\beta_{2}$ & 0 \\
$\beta_{3}$ & 0 \\
$\mathrm{t}_{1}$ & $\mathrm{~L}_{5}$ \\
$\mathrm{t}_{2}$ & $\mathrm{~L}_{6}$ \\
$\mathrm{t}_{3}$ & $-\mathrm{L}_{5}-\mathrm{L}_{6}$ \\
$\alpha_{1}$ & 0 \\
$\alpha_{2}$ & 0 \\
$\alpha_{3}$ & 0 \\
\hline
\end{tabular}


Quadro 25. Funções estimáveis do tipo III para efeitos de tratamentos no quadrado latino apresentado no quadro 12 , adotando a ordem tratamento, linha, coluna.

\begin{tabular}{cc}
\hline Parâmetros & Coeficientes \\
\hline$\mu$ & 0 \\
$\mathrm{t}_{1}$ & $\mathrm{~L}_{2}$ \\
$\mathrm{t}_{2}$ & $\mathrm{~L}_{3}$ \\
$\mathrm{t}_{3}$ & $-\mathrm{L}_{2}-\mathrm{L}_{3}$ \\
$\alpha_{1}$ & 0 \\
$\alpha_{2}$ & 0 \\
$\alpha_{3}$ & 0 \\
$\beta_{1}$ & 0 \\
$\beta_{2}$ & 0 \\
$\beta_{3}$ & 0 \\
\hline
\end{tabular}

\subsubsection{Funções estimáveis do tipo IV}

Analogamente aos casos anteriores, as funções estimáveis para o esquema do quadro 12 são as apresentadas nos quadros 26 a 29 . 
Quadro 26. Funções estimáveis do tipo IV para efeitos de tratamentos no quadrado latino apresentado no quadro 12 , adotando a ordem linha, coluna, tratamento.

\begin{tabular}{cc}
\hline Parâmetros & Coeficientes \\
\hline$\mu$ & 0 \\
$\alpha_{1}$ & 0 \\
$\alpha_{2}$ & 0 \\
$\alpha_{3}$ & 0 \\
$\beta_{1}$ & 0 \\
$\beta_{2}$ & 0 \\
$\beta_{3}$ & 0 \\
$\mathbf{t}_{1}$ & $\mathrm{~L}_{8}$ \\
$\mathbf{t}_{2}$ & $\mathrm{~L}_{9}$ \\
$\mathbf{t}_{3}$ & $-\mathrm{L}_{8}-\mathrm{L}_{9}$ \\
\hline
\end{tabular}


Quadro 27. Funções estimáveis do tipo IV para efeitos de tratamentos no quadrado latino apresentado no quadro 12, adotando a ordem linha, tratamento, coluna.

\begin{tabular}{cc}
\hline Parâmetros & Coeficientes \\
\hline$\mu$ & 0 \\
$\alpha_{1}$ & 0 \\
$\alpha_{2}$ & 0 \\
$\alpha_{3}$ & 0 \\
$\mathrm{t}_{1}$ & $\mathrm{~L}_{5}$ \\
$\mathrm{t}_{2}$ & $\mathrm{~L}_{6}$ \\
$\mathrm{t}_{3}$ & $-\mathrm{L}_{5}-\mathrm{L}_{6}$ \\
$\beta_{1}$ & 0 \\
$\beta_{2}$ & 0 \\
$\beta_{3}$ & 0 \\
\hline
\end{tabular}


Quadro 28. Funções estimáveis do tipo IV para efeitos de tratamentos no quadrado latino apresentado no quadro 12 , adotando a ordem coluna, tratamento, linha.

\begin{tabular}{cc}
\hline Parâmetros & Coeficientes \\
\hline$\mu$ & 0 \\
$\beta_{1}$ & 0 \\
$\beta_{2}$ & 0 \\
$\beta_{3}$ & 0 \\
$\mathrm{t}_{1}$ & $\mathrm{~L}_{5}$ \\
$\mathrm{t}_{2}$ & $\mathrm{~L}_{6}$ \\
$\mathrm{t}_{3}$ & $-\mathrm{L}_{5}-\mathrm{L}_{6}$ \\
$\alpha_{1}$ & 0 \\
$\alpha_{2}$ & 0 \\
$\alpha_{3}$ & 0 \\
\hline
\end{tabular}


Quadro 29. Funções estimáveis do tipo IV para efeitos de tratamentos no quadrado latino apresentado no quadro 12 , adotando a ordem tratamento, linha, coluna.

\begin{tabular}{cc}
\hline Parâmetros & Coeficientes \\
\hline$\mu$ & 0 \\
$\mathrm{t}_{1}$ & $\mathrm{~L}_{2}$ \\
$\mathrm{t}_{2}$ & $\mathrm{~L}_{3}$ \\
$\mathrm{t}_{3}$ & $-\mathrm{L}_{2}-\mathrm{L}_{3}$ \\
$\alpha_{1}$ & 0 \\
$\alpha_{2}$ & 0 \\
$\alpha_{3}$ & 0 \\
$\beta_{1}$ & 0 \\
$\beta_{2}$ & 0 \\
$\beta_{3}$ & 0 \\
\hline
\end{tabular}

Essas funções são similares às do tipo III quando não existem caselas vazias.

As hipóteses do tipo IV referem-se a contrastes entre médias de caselas.

Se ocorrer casela vazia, as somas de quadrados do tipo IV podem não ser únicas, pois dependem da posição e do número de caselas 
vazias. Se não há caselas vazias, as somas de quadrados dos tipos III e IV são iguais.

\subsection{Hipóteses Testadas}

Para simplificar a interpretação das hipóteses, tomou-se como base para o desenvolvimento deste tópico, o esquema do quadro 12 .

\subsubsection{Ordenação: Linha, Coluna, Tratamento}

Para esse tipo de ordenação, nos quatro tipos de somas de quadrados, tem-se a soma de quadrados de tratamentos dada por

$$
R(t \mid \mu, \alpha, \beta),
$$

que é a soma de quadrados associada à hipótese

$$
\mathrm{H}_{0}: \mathrm{t}_{1}=\mathrm{t}_{2}=\mathbf{t}_{3} \text {, }
$$

assim, com $K-1=2$ graus de liberdade podem-se tomar, dentre outras equivalentes, as formas :

$$
H_{0}:\left\{\begin{array}{l}
t_{1}=t_{2} \\
t_{1}=t_{3}
\end{array} \quad ; \quad H_{0}:\left\{\begin{array}{c}
t_{1}+t_{2}-2 t_{3}=0 \\
t_{1}-t_{2}=0
\end{array}\right.\right.
$$

e assim por diante. 
Neste caso, a hipótese é a mesma para os quatro tipos de somas de quadrados já descritos, pois para o tipo $\mathrm{I}$, a forma seqüencial ajusta naturalmente os tratamentos para linhas e colunas, conforme previsto por Yates (1936), citado por PEARCE (1976), para o caso balanceado. De certo modo fica aqui caracterizada a robustez do delineamento em quadrado latino para a falta de balanceamento.

Para o tipo Il, a hipótese é composta automaticamente de funções de tratamentos ajustados para linhas e colunas, o mesmo ocorrendo com os tipos III e IV, pois o modelo não contém interação. Assim, para essa ordem de entrada dos parâmetros no modelo, tem-se :

$$
\mathrm{H}_{0}^{(\mathrm{III})}=\mathrm{H}_{0}^{(\mathrm{III.1)}}=\mathrm{H}_{0}^{(\mathrm{IIII.1)}}=\mathrm{H}_{0}^{(\mathrm{IV} .1)}=\mathrm{H}_{0} .
$$

Nesse caso, as hipóteses dos tipos I a IV reduzem-se às hipóteses sobre médias não ponderadas de tratamentos.

Tomando-se, por exemplo, a forma :

$$
H_{0}^{(I .1)}:\left\{\begin{array}{l}
t_{1}=t_{3} \\
t_{2}=t_{3}
\end{array} \quad \text { ou } \quad H_{0}^{(I .1)}:\left\{\begin{array}{l}
t_{1}-t_{3}=0 \\
t_{2}-t_{3}=0
\end{array},\right.\right.
$$

vem, para a primeira linha de $\mathrm{H}_{0}^{(\mathrm{II.1})}: \mathrm{t}_{1}=\mathbf{t}_{3}$ : 
79.

$$
\begin{aligned}
& \mu+\alpha_{1}+\beta_{1}+\mathrm{t}_{1} \\
& \mu+\alpha_{2}+\beta_{2}+t_{1} \\
& \mu \quad+\alpha_{3} \quad+\beta_{3}+t_{1} \\
& \begin{array}{lll}
-\mu-\alpha_{1} & -\beta_{3} & -\mathrm{t}_{3}
\end{array} \\
& \begin{array}{llll}
-\mu & -\alpha_{2} & -\beta_{1} & -\mathrm{t}_{3}
\end{array} \\
& \begin{array}{llll}
-\mu & -\alpha_{3} & -\beta_{2} & -t_{3}
\end{array} \\
& 3 t_{1}-3 t_{3}
\end{aligned}
$$

que resulta $t_{1}-t_{3}=0$. De modo análogo, obtém-se para a segunda linha de $H_{0}^{(1.1)}: t_{2}-t_{3}=0$, mostrando que a hipótese testada é :

$$
H_{0}:\left\{\begin{array}{l}
t_{1}-t_{3}=0 \\
t_{2}-t_{3}=0
\end{array}\right.
$$

Este fato pode ser também observado, nos quadros das funções estimáveis, bem como nos quadros que exibem os quatro tipos de somas de quadrados.

\subsubsection{Ordenação: Linha, Tratamento, Coluna}

Conforme visto nos quadros de funções estimáveis, para as condições do exemplo em questão: 


$$
\mathrm{H}_{0}^{(\mathrm{d} .2)}:\left\{\begin{array}{l}
\mathrm{t}_{1}-\mathrm{t}_{3}-0,2098 \beta_{1}+0,2910 \beta_{2}-0,0811 \beta_{3}=0 \\
t_{2}-t_{3}-0,0720 \beta_{1}-0,0652 \beta_{2}+0,1373 \beta_{3}=0
\end{array},\right.
$$

que é, sem dúvida, uma hipótese de difícil interpretação, evidenciando o fato de que a ordem de entrada dos parâmetros no modelo, deve reservar a última posição para tratamentos.

Para as demais hipóteses, tem-se, como no item (3.7.1) :

$$
\mathrm{H}_{0}^{(\mathrm{II} .2)}=\mathrm{H}_{0}^{(\mathrm{III} .2)}=\mathrm{H}_{0}^{(\mathrm{IV} \cdot 2)}=\mathrm{H}_{0}
$$

Tais fatos podem ser observados de forma mais detalhada nos quadros do exemplo ilustrativo, no capítulo a seguir .

\subsubsection{Ordenação: Coluna, Tratamento, Linha}

De forma análoga às anteriores, tem-se para a hipótese tịpo I :

$$
H_{0}^{(1.3)}:\left\{\begin{array}{l}
t_{1}-t_{3}-0,0538 \alpha_{1}+0,3281 \alpha_{2}-0,2743 \alpha_{3}=0 \\
t_{2}-t_{3}-0,0353 \alpha_{1}+0,0925 \alpha_{2}-0,0573 \alpha_{3}=0
\end{array}\right.
$$

e, para as demais hipóteses :

$$
\mathrm{H}_{0}^{(\mathrm{III} .3)}=\mathrm{H}_{0}^{(\mathrm{III} \cdot 3)}=\mathrm{H}_{0}^{(\mathrm{IV} \cdot 3)}=\mathrm{H}_{0}
$$

conforme pode-se observar no exemplo ilustrativo. 


\subsubsection{Ordenação: Tratamento, Linha, Coluna}

Neste caso, analogamente aos anteriores, tem-se para a hipótese tipo I :

$$
H_{0}^{(I .4)}:\left\{\begin{array}{c}
t_{1}-t_{2}-0,0667 \alpha_{1}+0,2667 \alpha_{2}-0,2000 \alpha_{3} \\
-0,1333 \beta_{1}+0,2000 \beta_{2}-0,0667 \beta_{3}=0 \\
t_{2}-t_{3}+0,0190 \alpha_{1}+0,0952 \alpha_{2}-0,1143 \alpha_{3} \\
-0,0476 \beta_{1}-0,1143 \beta_{2}+0,1619 \beta_{3}=0,
\end{array}\right.
$$

e para as demais hipóteses :

$$
\mathrm{H}_{0}^{(\mathrm{III} .4)}=\mathrm{H}_{0}^{(\mathrm{IIII.4)}}=\mathrm{H}_{0}^{(\mathrm{IV} .4)}=\mathrm{H}_{0}
$$

conforme o exemplo ilustrativo.

Salienta-se que o objetivo final de experimentos com delineamentos quadrados latinos é testar o fator tratamentos, pois linhas e colunas são apenas fatores de restrição na casualização. No entanto, as mesmas somas de quadrados são fornecidas pelo SAS para os delineamentos com três fatores sem interação, pois, nesses casos linhas e colunas são também fatores a serem testados e a interpretação das hipóteses correspondentes pode ser feita de modo análogo ao aqui exposto.

\subsection{Tipos de Somas de Quadrados}

Os quatro tipos de somas de quadrados discutidas são: 


\subsubsection{Somas de quadrados do tipo I}

As somas de quadrados do tipo I são obtidas de acordo com a ordem dos fatores no modelo, pois são seqüenciais, ou seja, cada parâmetro é ajustado para todos os anteriores. Sendo assim, este tipo de soma de quadrados testa hipóteses sobre médias ponderadas não ajustadas.

No caso do quadrado latino com número diferente de observações por unidade experimental, de acordo com algumas ordenações, dando ênfase para tratamento, que é o fator de interesse para o delineamento, obtém-se :

\section{a) Ordenạ̧ão: Linha, Coluna, Tratamento}

Quadro 30. Somas de quadrados tipo I para a ordenação linha, coluna, tratamento.

\begin{tabular}{lcll}
\hline Causas de Variação & G.L. & \multicolumn{1}{c}{$\mathrm{R}(\mathrm{)})$} & Hipótese testada \\
\hline Linhas (não ajustada) & $\mathrm{I}-1$ & $\mathrm{R}(\alpha \mid \mu)$ & \\
Colunas (ajustada) & $\mathrm{J}-1$ & $\mathrm{R}(\beta \mid \mu, \alpha)$ & \\
Tratamentos (ajustada) & $\mathrm{K}-1$ & $\mathrm{R}(\mathrm{t} \mid \mu, \alpha, \beta)$ & $\mathrm{H}_{0}^{(\mathrm{III})}$ \\
\hline
\end{tabular}




\section{b) Ordenação: Linha, Tratamento, Coluna}

Quadro 31. Somas de quadrados tipo I para a ordenação linha, tratamento, coluna.

\begin{tabular}{lclc}
\hline Causas de Variação & G.L. & \multicolumn{1}{c}{$\mathrm{R}()$} & Hipótese testada \\
\hline Linhas (não ajustada) & $\mathrm{I}-1$ & $\mathrm{R}(\alpha \mid \mu)$ & \\
Tratamentos (ajustada) & $\mathrm{K}-1$ & $\mathrm{R}(\mathrm{t} \mid \mu, \alpha)$ & $\mathrm{H}_{0}^{(\mathrm{I} .2)}$ \\
Colunas (ajustada) & $\mathrm{J}-1$ & $\mathrm{R}(\beta \mid \mu, \alpha, \mathrm{t})$ & \\
\hline
\end{tabular}

\section{c) Ordenação: Coluna, Tratamento, Linha}

Quadro 32. Somas de quadrados tipo I para a ordenação coluna, tratamento, linha.

\begin{tabular}{lclc}
\hline Causas de Variação & G.L. & \multicolumn{1}{c}{$\mathrm{R}()$} & Hipótese testada \\
\hline Colunas (não ajustada) & $\mathrm{J}-1$ & $\mathrm{R}(\boldsymbol{\beta} \mid \mu)$ & \\
Tratamentos (ajustada) & $\mathrm{K}-1$ & $\mathrm{R}(\mathrm{t} \mid \mu, \beta)$ & $\mathrm{H}_{0}^{(1.3)}$ \\
Linhas (ajustada) & $\mathrm{I}-1$ & $\mathrm{R}(\alpha \mid \mu, \beta, \mathrm{t})$ & \\
\hline
\end{tabular}




\section{d) Ordenação: Tratamento, Linha, Coluna}

Quadro 33. Somas de quadrados tipo I para a ordenação tratamento, linha, coluna.

\begin{tabular}{lclc}
\hline Causas de Variação & G.L. & \multicolumn{1}{c}{$\mathrm{R}(\mathrm{O})$} & Hipótese testada \\
\hline Tratamentos (não ajustada) & $\mathrm{K}-1$ & $\mathrm{R}(\mathrm{t} \mid \mu)$ & $\mathrm{H}_{0}^{(\mathrm{I} .4)}$ \\
Linhas (ajustada) & $\mathrm{I}-1$ & $\mathrm{R}(\alpha \mid \mu, \mathrm{t})$ & \\
Colunas (ajustada) & $\mathrm{J}-1$ & $\mathrm{R}(\beta \mid \mu, \alpha, \mathrm{t})$ & \\
\hline
\end{tabular}

Salienta-se que, no caso de somas de quadrados do tipo I, por exemplo no quadro 33 :

$$
\mathrm{R}(\mu)+\mathrm{R}(\mathrm{t} \mid \mu)+\mathrm{R}(\alpha \mid \mu, \mathrm{t})+\mathrm{R}(\beta \mid \mu, \alpha, \mathrm{t})=\mathrm{R}(\mu, \alpha, \beta, \mathrm{t})=\text { S.Q.Parâmetros ; }
$$

fato este que também ocorre para os quadros 30 a 32 .

\subsubsection{Somas de quadrados do tipo II}

As somas de quadrados tipo II podem ser obtidas das somas de quadrados do tipo I, escolhendo-se somente as hipóteses sobre médias ponderadas ajustadas.

Para estas somas de quadrados, no caso do quadrado latino em estudo, de acordo com a ordenação dos fatores, obtém-se : 


\section{a) Ordenação: Linha, Coluna, Tratamento}

Quadro 34. Somas de quadrados tipo II para a ordenação linha, coluna, tratamento.

\begin{tabular}{lccc}
\hline Causas de Variação & G.L. & $\mathrm{R}(\mathrm{)})$ & Hipótese testada \\
\hline Linhas (ajustada) & $\mathrm{I}-1$ & $\mathrm{R}(\alpha \mid \mu, \beta, \mathrm{t})$ & \\
Colunas (ajustada) & $\mathrm{J}-1$ & $\mathrm{R}(\beta \mid \mu, \alpha, \mathrm{t})$ & \\
Tratamentos (ajustada) & $\mathrm{K}-1$ & $\mathrm{R}(\mathrm{t} \mid \mu, \alpha, \beta)$ & $\mathrm{H}_{0}^{(\mathrm{III} .1)}$ \\
\hline
\end{tabular}

b) Ordenação: Linha, Tratamento, Coluna

Quadro 35. Somas de quadrados tipo II para a ordenação linha, tratamento, coluna.

\begin{tabular}{lccc}
\hline Causas de Variação & G.L. & $\mathrm{R}()$ & Hipótese testada \\
\hline Linhas (ajustada) & $\mathrm{I}-1$ & $\mathrm{R}(\alpha \mid \mu, \beta, \mathrm{t})$ & \\
Tratamentos (ajustada) & $\mathrm{K}-1$ & $\mathrm{R}(\mathrm{t} \mid \mu, \alpha, \beta)$ & $\mathrm{H}_{0}^{(\mathrm{II} .2)}$ \\
Colunas (ajustada) & $\mathrm{J}-1$ & $\mathrm{R}(\beta \mid \mu, \alpha, \mathrm{t})$ & \\
\hline
\end{tabular}




\section{c) Ordenação: Coluna, Tratamento, Linha}

Quadro 36. Somas de quadrados tipo II para a ordenação coluna, tratamento, linha.

\begin{tabular}{lccc}
\hline Causas de Variação & G.L. & $\mathrm{R}()$ & Hipótese testada \\
\hline Colunas (ajustada) & $\mathrm{J}-1$ & $\mathrm{R}(\beta \mid \mu, \alpha, \mathrm{t})$ & \\
Tratamentos (ajustada) & $\mathrm{K}-1$ & $\mathrm{R}(\mathrm{t} \mid \mu, \alpha, \beta)$ & $\mathrm{H}_{0}^{(\mathrm{II} .3)}$ \\
Linhas (ajustada) & $\mathrm{I}-1$ & $\mathrm{R}(\alpha \mid \mu, \beta, \mathrm{t})$ & \\
\hline
\end{tabular}

\section{d) Ordenação: Tratamento, Linha, Coluna}

Quadro 37. Somas de quadrados tipo II para a ordenação tratamento, linha, coluna.

\begin{tabular}{lccc}
\hline Causas de Variação & G.L. & $\mathrm{R}()$ & Hipótese testada \\
\hline Tratamentos (ajustada) & $\mathrm{K}-1$ & $\mathrm{R}(\mathrm{t} \mid \mu, \alpha, \beta)$ & $\mathrm{H}_{0}^{(\mathrm{II} .4)}$ \\
Linhas (ajustada) & $\mathrm{I}-1$ & $\mathrm{R}(\alpha \mid \mu, \beta, \mathrm{t})$ & \\
Colunas (ajustada) & $\mathrm{J}-1$ & $\mathrm{R}(\beta \mid \mu, \alpha, \mathrm{t})$ & \\
\hline
\end{tabular}

Neste caso, nem sempre as somas de quadrados são provenientes de uma partição ortogonal da S.Q.Parâmetros, o que não ocorre para as do tipo I, conforme já foi comentado, pois aqui, se for tomado como exemplo o quadro 34 , tem-se : 


$$
R(\mu)+R(\alpha \mid \mu, \beta, t)+R(\beta \mid \mu, \alpha, t)=R(t \mid \mu, \alpha, \beta) \neq S \text {.Q.Parâmetros ; }
$$

fato este que tambem ocorre para os quadros 35 a 37 .

\subsubsection{Somas de quadrados do tipo III}

Para o delineamento em estudo, ou seja, no caso de desbalanceamento com todas as caselas ocupadas e modelo sem interação, que de acordo com SEARLE (1987) é o caso do quadrado latino, as somas de quadrados do tipo III são iguais às do tipo II e suas hipóteses sobre médias não ponderadas e sobre médias ponderadas coincidem.

\subsubsection{Somas de quadrados do tipo IV}

As somas de quadrados do tipo IV, neste caso também são as mesmas dos tipos II e III, pois, pela própria característica do delineamento, classificado por SEARLE (1987) como um caso de desbalancemento planejado, não tem caselas vazias que é o que poderia mudar as somas de quadrados do tipo IV .

No caso de modelos com interação ou no caso de ocorrência de caselas vazias, podem-se obter maiores informações em IEMMA (1995c). 


\section{EXEMPLO ILUSTRATIVO}

Com o objetivo de ilustrar os resultados obtidos no desenvolvimento teórico, apresenta-se um exemplo prático considerando dados de teor de nitrogênio (\%) em cana-de-açúcar, variedade CB41-76, para cana-planta.

Os dados foram coletados na Estação Experimental do IAA/PLANALSUCAR - Araras - S.P..

Constam do experimento os seguintes tipos de solo (tratamentos) :

$\mathrm{LR}=$ Latossol Roxo;

$\mathrm{LE}=$ Latossol Vermelho Escuro - Orto;

PVls $=$ Podzólico Vermelho Amarelo Var. Laras.

O experimento foi instalado no delineamento em quadrado latino $3 \times 3$, considerando como linhas as partes da planta: colmo, folha $\mathrm{e}$ folha +3 ; como colunas as idades das plantas: 4,10 e 16 meses .

Os dados coletados no experimento são: 
Quadro 38. Dados referentes à concentração de nitrogênio, em \%, obtidos em um experimento com cana-de-açúcar, cujo delineamento é quadrado latino com número diferente de observações por unidade experimental.

\begin{tabular}{|c|c|c|c|c|c|c|}
\hline \multirow{2}{*}{$\begin{array}{l}\text { Partes } \\
\text { da } \\
\text { Planta }\end{array}$} & \multicolumn{6}{|c|}{$\begin{array}{l}\text { Idades } \\
\text { (meses) }\end{array}$} \\
\hline & \multicolumn{2}{|c|}{4} & \multicolumn{2}{|c|}{10} & \multicolumn{2}{|c|}{16} \\
\hline Colmo & LR & 1,64 & LE & $\begin{array}{l}0,62 \\
0,61\end{array}$ & PVls & $\begin{array}{l}0,41 \\
0,39 \\
0,37 \\
0,38\end{array}$ \\
\hline Folha & PVls & $\begin{array}{l}1,11 \\
1,09 \\
1,10 \\
1,12 \\
1,11\end{array}$ & LR & $\begin{array}{l}0,81 \\
0,80 \\
0,82\end{array}$ & LE & $\begin{array}{l}0,62 \\
0,62 \\
0,61\end{array}$ \\
\hline Folha +3 & LE & $\begin{array}{l}2,13 \\
2,11\end{array}$ & PVls & $\begin{array}{l}1,86 \\
1,88 \\
1,85 \\
1,84 \\
1,84 \\
1,80\end{array}$ & LR & 1,43 \\
\hline
\end{tabular}

Procedendo de acordo com o desenvolvimento teórico, descrito anteriormente, obtiveram-se os seguintes resultados:

4.1. Análises da Variância Segundo os Tipos de Somas de Quadrados e Ordenações dos Fatores no Modelo. 


\subsubsection{Ordenação: Linha, Coluna, Tratamento}

Quadro 39. Análise da variância dos dados do quadro 38 para a ordenação linha, coluna, tratamento.

Tipo I

\begin{tabular}{|c|c|c|c|c|}
\hline Causas de Variação & G.L. & $\mathrm{R}(\mathrm{)}$ & Hipótese testada & S.Q. \\
\hline Linhas & 2 & $\mathrm{R}(\alpha \mid \mu)$ & & 7,1523 \\
\hline Colunas & 2 & $R(\beta \mid \mu, \alpha)$ & & 1,6497 \\
\hline Tratamentos & 2 & $\mathrm{R}(\mathrm{t} \mid \mu, \alpha, \beta)$ & $\mathrm{H}_{0}^{(\mathrm{III})}$ & 0,0607 \\
\hline \multicolumn{5}{|c|}{ Tipo II } \\
\hline Causas de Variação & G.L. & $\mathrm{R}(\mathrm{)}$ & Hipótese testada & S.Q. \\
\hline Linhas & 2 & $\mathrm{R}(\alpha \mid \mu, \beta, \mathrm{t})$ & & 5,3885 \\
\hline Colunas & 2 & $\mathrm{R}(\beta \mid \mu, \alpha, \mathrm{t})$ & & 1,6713 \\
\hline Tratamentos & 2 & $\mathrm{R}(\mathrm{t} \mid \boldsymbol{\mu}, \alpha, \beta)$ & $\mathrm{H}_{0}^{(\mathrm{III.1)}}$ & 0,0607 \\
\hline \multicolumn{5}{|c|}{ Tipo III } \\
\hline Causas de Variação & G.L. & $\mathrm{R}(\mathrm{)}$ & Hipótese testada & S.Q. \\
\hline Linhas & 2 & $\mathrm{R}(\alpha \mid \mu, \beta, \mathrm{t})$ & & 5,3885 \\
\hline Colunas & 2 & $\mathrm{R}(\beta \mid \mu, \alpha, t)$ & & 1,6713 \\
\hline Tratamentos & 2 & $\mathrm{R}(\mathrm{t} \mid \mu, \alpha, \beta)$ & $\mathrm{H}_{0}^{(\mathrm{IIII} .1)}$ & 0,0607 \\
\hline \multicolumn{5}{|c|}{ Tipo IV } \\
\hline Causas de Variação & G.L. & $\mathrm{R}(\mathbf{)}$ & Hipótese testada & S.Q. \\
\hline Linhas & 2 & $\mathrm{R}(\alpha \mid \mu, \beta, \mathrm{t})$ & & 5,3885 \\
\hline Colunas & 2 & $\mathrm{R}(\beta \mid \mu, \alpha, \mathrm{t})$ & & 1,6713 \\
\hline Tratamentos & 2 & $\mathrm{R}(\mathrm{t} \mid \boldsymbol{\mu}, \alpha, \beta)$ & $\mathrm{H}_{0}^{(\mathrm{IV} .1)}$ & 0,0607 \\
\hline
\end{tabular}




\subsubsection{Ordenação: Linha, Tratamento, Coluna}

Quadro 40. Análise da variância dos dados do quadro 38 para a ordenação linha, tratamento, coluna.

Tipo I

\begin{tabular}{|c|c|c|c|c|}
\hline Causas de Variação & G.L. & $\mathrm{R}()$ & Hipótese testada & S.Q. \\
\hline Linhas & 2 & $\mathrm{R}(\alpha \mid \mu)$ & \multirow{3}{*}{$\mathrm{H}_{0}^{(1.2)}$} & 7,1523 \\
\hline Tratamentos & 2 & $R(t \mid \mu, \alpha)$ & & 0,0391 \\
\hline Colunas & 2 & $R(\beta \mid \mu, \alpha, t)$ & & 1,6713 \\
\hline \multicolumn{5}{|c|}{ Tipo II } \\
\hline Causas de Variação & G.L. & $\mathrm{R}()$ & Hipótese testada & S.Q. \\
\hline Linhas & 2 & $\mathrm{R}(\alpha \mid \boldsymbol{\mu}, \beta, \mathrm{t})$ & \multirow{3}{*}{$\mathrm{H}_{0}^{(\mathrm{II} .2)}$} & 5,3885 \\
\hline Tratamentos & 2 & $\mathrm{R}(\mathrm{t} \mid \mu, \alpha, \beta)$ & & 0,0607 \\
\hline Colunas & 2 & $\mathrm{R}(\boldsymbol{\beta} \mid \boldsymbol{\mu}, \alpha, \mathrm{t})$ & & 1,6713 \\
\hline \multicolumn{5}{|c|}{ Tipo III } \\
\hline Causas de Variação & G.L. & $\mathrm{R}()$ & Hipótese testada & S.Q. \\
\hline Linhas & 2 & $\mathrm{R}(\alpha \mid \mu, \beta, \mathfrak{t})$ & \multirow{3}{*}{$\mathrm{H}_{0}^{(\mathrm{IIII} .2)}$} & 5,3885 \\
\hline Tratamentos & 2 & $\mathrm{R}(\mathrm{t} \mid \mu, \alpha, \beta)$ & & 0,0607 \\
\hline Colunas & 2 & $R(\beta \mid \mu, \alpha, t)$ & & 1,6713 \\
\hline \multicolumn{5}{|c|}{ Tipo IV } \\
\hline Causas de Variação & G.L. & $\mathrm{R}()$ & Hipótese testada & S.Q. \\
\hline Linhas & 2 & $\mathrm{R}(\alpha \mid \mu, \beta, \mathrm{t})$ & \multirow{3}{*}{$\mathrm{H}_{0}^{(\mathrm{IV} .2)}$} & 5,3885 \\
\hline Tratamentos & 2 & $\mathrm{R}(\mathfrak{t} \mid \mu, \alpha, \beta)$ & & 0,0607 \\
\hline Colunas & 2 & $R(\beta \mid \mu, \alpha, t)$ & & 1,6713 \\
\hline
\end{tabular}




\subsubsection{Ordenação: Coluna, Tratamento, Linha}

Quadro 41. Análise da variância dos dados do quadro 38 para a ordenação coluna, tratamento, linha.

Tipo I

\begin{tabular}{|c|c|c|c|c|}
\hline Causas de Variação & G.L. & $\mathrm{R}(\mathrm{)}$ & Hipótese testada & S.Q. \\
\hline Colunas & 2 & $\mathrm{R}(\boldsymbol{\beta} \mid \boldsymbol{\mu})$ & \multirow{3}{*}{$\mathrm{H}_{0}^{(1.3)}$} & 3,3907 \\
\hline Tratamentos & 2 & $\mathrm{R}(\mathrm{t} \mid \mu, \beta)$ & & 0,0835 \\
\hline Linhas & 2 & $\mathrm{R}(\alpha \mid \mu, \beta, \mathrm{t})$ & & 5,3885 \\
\hline \multicolumn{5}{|c|}{ Tipo II } \\
\hline Causas de Variação & G.L. & $\mathrm{R}()$ & Hipótese testada & S.Q. \\
\hline Colunas & 2 & $\mathrm{R}(\beta \mid \mu, \alpha, \mathrm{t})$ & \multirow{3}{*}{$\mathrm{H}_{0}^{(\mathrm{II} \cdot 3)}$} & 1,6713 \\
\hline Tratamentos & 2 & $\mathrm{R}(\mathrm{t} \mid \boldsymbol{\mu}, \boldsymbol{\alpha}, \boldsymbol{\beta})$ & & 0,0607 \\
\hline Linhas & 2 & $\mathrm{R}(\alpha \mid \mu, \beta, \mathrm{t})$ & & 5,3885 \\
\hline \multicolumn{5}{|c|}{ Tipo III } \\
\hline Causas de Variação & G.L. & $\mathrm{R}()$ & Hipótese testada & S.Q. \\
\hline Colunas & 2 & $\mathrm{R}(\beta \mid \mu, \alpha, \mathrm{t})$ & \multirow{3}{*}{$\mathrm{H}_{0}^{(\mathrm{IIII.3)}}$} & 1,6713 \\
\hline Tratamentos & 2 & $\mathrm{R}(\mathrm{t} \mid \mu, \alpha, \beta)$ & & 0,0607 \\
\hline Linhas & 2 & $\mathrm{R}(\alpha \mid \mu, \beta, \mathrm{t})$ & & 5,3885 \\
\hline \multicolumn{5}{|c|}{ Tipo IV } \\
\hline Causas de Variação & G.L. & $\mathrm{R}(\mathbf{)}$ & Hipótese testada & S.Q. \\
\hline Colunas & 2 & $\mathrm{R}(\beta \mid \mu, \alpha, \mathrm{t})$ & \multirow{3}{*}{$\mathrm{H}_{0}^{(\mathrm{IV} \cdot 3)^{\prime}}$} & 1,6713 \\
\hline Tratamentos & 2 & $\mathrm{R}(\mathrm{t} \mid \mu, \alpha, \beta)$ & & 0,0607 \\
\hline Linhas & 2 & $\mathrm{R}(\alpha \mid \mu, \beta, t)$ & & 5,3885 \\
\hline
\end{tabular}




\subsubsection{Ordenação: Tratamento, Linha, Coluna}

Quadro 42. Análise da variância dos dados do quadro 38 para a ordenação tratamento, linha, coluna.

Tipo I

\begin{tabular}{|c|c|c|c|c|}
\hline Causas de Variação & G.L. & $\mathrm{R}()$ & Hipótese testada & S.Q. \\
\hline Tratamentos & 2 & $\mathrm{R}(\mathrm{t} \mid \mu)$ & $\mathrm{H}_{0}^{(1.4)}$ & 0,1424 \\
\hline Linhas & 2 & $\mathrm{R}(\alpha \mid \mu, \mathrm{t})$ & & 7,0490 \\
\hline Colunas & 2 & $R(\beta \mid \mu, \alpha, t)$ & & 1,6713 \\
\hline \multicolumn{5}{|c|}{ Tipo II } \\
\hline Causas de Variação & G.L. & $\mathrm{R}()$ & Hipótese testada & S.Q. \\
\hline Tratamentos & 2 & $\mathrm{R}(\mathrm{t} \mid \boldsymbol{\mu}, \boldsymbol{\alpha}, \boldsymbol{\beta})$ & $\mathrm{H}_{0}^{(\mathrm{III} .4)}$ & 0,0607 \\
\hline Linhas & 2 & $\mathrm{R}(\alpha \mid \mu, \beta, \mathrm{t})$ & & 5,3885 \\
\hline Colunas & 2 & $\mathrm{R}(\beta \mid \mu, \alpha, \mathrm{t})$ & & 1,6713 \\
\hline \multicolumn{5}{|c|}{ Tipo III } \\
\hline Causas de Variação & G.L. & $\mathrm{R}()$ & Hipótese testada & S.Q. \\
\hline Tratamentos & 2 & $\mathrm{R}(\mathrm{t} \mid \mu, \alpha, \beta)$ & $\mathrm{H}_{0}^{(\mathrm{IIII} .4)}$ & 0,0607 \\
\hline Linhas & 2 & $\mathrm{R}(\alpha \mid \mu, \beta, \mathrm{t})$ & & 5,3885 \\
\hline Colunas & 2 & $\mathrm{R}(\beta \mid \mu, \alpha, \mathrm{t})$ & & 1,6713 \\
\hline \multicolumn{5}{|c|}{ Tipo IV } \\
\hline Causas de Variação & G.L. & $\mathrm{R}(\mathrm{)}$ & Hipótese testada & S.Q. \\
\hline Tratamentos & 2 & $\mathrm{R}(\mathrm{t} \mid \mu, \alpha, \beta)$ & $\mathrm{H}_{0}^{(\mathrm{IV} .4)}$ & 0,0607 \\
\hline Linhas & 2 & $\mathrm{R}(\alpha \mid \mu, \beta, \mathrm{t})$ & & 5,3885 \\
\hline Colunas & 2 & $\mathrm{R}(\beta \mid \mu, \alpha, t)$ & & 1,6713 \\
\hline
\end{tabular}




\subsection{Funções Estimáveis para Tratamentos}

\subsubsection{Ordenação: Linha, Coluna, Tratamento}

Quadro 43. Funções estimáveis de tratamentos para a ordenação linha, coluna, tratamento.

\begin{tabular}{cc}
\hline Parâmetros & Coeficientes (tipos I, II, III e IV) \\
\hline$\mu$ & 0 \\
$\alpha_{1}$ & 0 \\
$\alpha_{2}$ & 0 \\
$\alpha_{3}$ & 0 \\
$\beta_{1}$ & 0 \\
$\beta_{2}$ & 0 \\
$\beta_{3}$ & 0 \\
$\mathrm{t}_{1}$ & L8 \\
$\mathrm{t}_{2}$ & L9 \\
$\mathrm{t}_{3}$ & -L8-L9 \\
\hline
\end{tabular}

Portanto, como já foi visto anteriormente :

$$
\mathrm{H}_{0}^{(\mathrm{III)})}=\mathrm{H}_{0}^{(\mathrm{III.1)}}=\mathrm{H}_{0}^{(\mathrm{IIII.1)}}=\mathrm{H}_{0}^{(\mathrm{IV} . \mathrm{I})}=\mathrm{H}_{0} \text {, }
$$

onde $H_{0}: t_{1}=t_{2}=t_{3}$.

ou, dentre outras equivalentes, as formas :

$$
H_{0}:\left\{\begin{array}{l}
t_{1}=t_{2} \\
t_{1}=t_{3}
\end{array} \quad ; \quad H_{0}:\left\{\begin{array}{c}
t_{1}+t_{2}-2 t_{3}=0 \\
t_{1}-t_{2}=0
\end{array}\right.\right.
$$




\subsubsection{Ordenação: Linha, Tratamento, Coluna}

Quadro 44. Funções estimáveis de tratamentos para a ordenação linha, tratamento, coluna.

\begin{tabular}{ccc}
\hline Parâmetros & Coeficientes (tipo I) & Coeficientes (tipos II, III e IV) \\
\hline$\mu$ & 0 & 0 \\
$\alpha_{1}$ & 0 & 0 \\
$\alpha_{2}$ & 0 & 0 \\
$\alpha_{3}$ & 0 & 0 \\
$\mathrm{t}_{1}$ & $\mathrm{~L} 5$ & $\mathrm{~L} 5$ \\
$\mathrm{t}_{2}$ & $\mathrm{~L} 6$ & $\mathrm{~L} 6$ \\
$\mathrm{t}_{3}$ & $-\mathrm{L} 5-\mathrm{L} 6$ & $-\mathrm{L} 5-\mathrm{L} 6$ \\
$\beta_{1}$ & $-0,2098 \mathrm{~L} 5-0,0720 \mathrm{~L} 6$ & 0 \\
$\beta_{2}$ & $0,2910 \mathrm{~L} 5-0,0652 \mathrm{~L} 6$ & 0 \\
$\beta_{3}$ & $-0,0811 \mathrm{~L} 5+0,1373 \mathrm{~L} 6$ & 0 \\
\hline
\end{tabular}

Assim, fazendo $\mathrm{L}_{5}=1$ e $\mathrm{L}_{6}=0$ e a seguir $\mathrm{L}_{5}=0$ e $\mathrm{L}_{6}=1$, obtém-se:

$$
H_{0}^{(1.2)}:\left\{\begin{array}{l}
t_{1}-t_{3}-0,2098 \beta_{1}+0,2910 \beta_{2}-0,0811 \beta_{3}=0 \\
t_{2}-t_{3}-0,0720 \beta_{1}-0,0652 \beta_{2}+0,1373 \beta_{3}=0
\end{array},\right.
$$

que é, sem dúvida, uma hipótese de dificil interpretação.

Para as demais hipóteses, tem-se, como no item (3.7.1) :

$$
\mathrm{H}_{0}^{(\mathrm{II.2} .2)}=\mathrm{H}_{0}^{(\mathrm{III} .2)}=\mathrm{H}_{0}^{(\mathrm{IV} .2)}=\mathrm{H}_{0}
$$


96.

\subsubsection{Ordenação: Coluna, Tratamento, Linha}

Quadro 45. Funções estimáveis de tratamentos para a ordenação coluna, tratamento, linha.

\begin{tabular}{ccc}
\hline Parâmetros & Coeficientes (tipo I) & Coeficientes (tipos II , III e IV) \\
\hline$\mu$ & 0 & 0 \\
$\beta_{1}$ & 0 & 0 \\
$\beta_{2}$ & 0 & 0 \\
$\beta_{3}$ & 0 & 0 \\
$\mathrm{t}_{1}$ & $\mathrm{~L} 5$ & $\mathrm{~L} 5$ \\
$\mathrm{t}_{2}$ & $\mathrm{~L} 6$ & $\mathrm{~L} 6$ \\
$\mathrm{t}_{3}$ & $-\mathrm{L} 5-\mathrm{L} 6$ & $-\mathrm{L} 5-\mathrm{L} 6$ \\
$\alpha_{1}$ & $-0,0538 \mathrm{~L} 5-0,0353 \mathrm{~L} 6$ & 0 \\
$\alpha_{2}$ & $0,3281 \mathrm{~L} 5+0,0925 \mathrm{~L} 6$ & 0 \\
$\alpha_{3}$ & $-0,2743 \mathrm{~L} 5-0,0573 \mathrm{~L} 6$ & 0 \\
\hline
\end{tabular}

De forma análoga às anteriores, tem-se para a hipótese tipo I :

$$
H_{0}^{(1.3)}:\left\{\begin{array}{l}
t_{1}-t_{3}-0,0538 \alpha_{1}+0,3281 \alpha_{2}-0,2743 \alpha_{3}=0 \\
t_{2}-t_{3}-0,0353 \alpha_{1}+0,0925 \alpha_{2}-0,0573 \alpha_{3}=0
\end{array}\right.
$$

e, para as demais hipóteses :

$$
\mathrm{H}_{0}^{(\mathrm{II} .3)}=\mathrm{H}_{0}^{(\mathrm{III} .3)}=\mathrm{H}_{0}^{(\mathrm{IV} .3)}=\mathrm{H}_{0}
$$




\subsubsection{Ordenação: Tratamento, Linha, Coluna}

Quadro 46. Funções estimáveis de tratamentos para a ordenação tratamento, linha, coluna.

\begin{tabular}{ccc}
\hline Parâmetros & Coeficientes (tipo I) & Coeficientes (tipos II, III e IV) \\
\hline$\mu$ & 0 & 0 \\
$t_{1}$ & L2 & L2 \\
$t_{2}$ & L3 & L3 \\
$t_{3}$ & $-\mathrm{L} 2-\mathrm{L} 3$ & $-\mathrm{L} 2-\mathrm{L} 3$ \\
$\alpha_{1}$ & $-0,0667 \mathrm{~L} 2-0,0190 \mathrm{~L} 3$ & 0 \\
$\alpha_{2}$ & $0,2667 \mathrm{~L} 2+0,0952 \mathrm{~L} 3$ & 0 \\
$\alpha_{3}$ & $-0,2000 \mathrm{~L} 2-0,1143 \mathrm{~L} 3$ & 0 \\
$\beta_{1}$ & $-0,1333 \mathrm{~L} 2-0,0476 \mathrm{~L} 3$ & 0 \\
$\beta_{2}$ & $0,2000 \mathrm{~L} 2-0,1143 \mathrm{~L} 3$ & 0 \\
$\beta_{3}$ & $-0,0667 \mathrm{~L} 2+0,1619 \mathrm{~L} 3$ & 0 \\
\hline
\end{tabular}

Neste caso, analogamente aos anteriores, tem-se para a hipótese tipo I :

$$
H_{0}^{(1.4)}:\left\{\begin{array}{c}
t_{1}-t_{2}-0,0667 \alpha_{1}+0,2667 \alpha_{2}-0,2000 \alpha_{3} \\
-0,1333 \beta_{1}+0,2000 \beta_{2}-0,0667 \beta_{3}=0 \\
t_{2}-t_{3}+0,0190 \alpha_{1}+0,0952 \alpha_{2}-0,1143 \alpha_{3} \\
-0,0476 \beta_{1}-0,1143 \beta_{2}+0,1619 \beta_{3}=0
\end{array},\right.
$$

e, para as demais hipóteses :

$$
\mathrm{H}_{0}^{(\mathrm{II} .4)}=\mathrm{H}_{0}^{(\mathrm{III} .4)}=\mathrm{H}_{0}^{(\mathrm{IV} .4)}=\mathrm{H}_{0}
$$


98.

No apêndice desse trabalho estão o programa e as saídas obtidas no SAS para o exemplo em questão.

Salienta-se que, para completar a análise estatística de seus dados, o pesquisador pode optar por um tipo de confronto entre tratamentos, comparando-os de forma mais detalhada, através de contrastes ou de métodos de comparações múltiplas, que o próprio pacote estatístico que ele estiver usando possibilita. No caso do SAS, seu manual (1990) traz várias opções.

O uso de outros pacotes estatísticos para a análise de dados desbalanceados pode ser encontrado em SANTOS (1994). 


\section{CONCLUSÕES}

Com base nos resultados pode-se concluir:

1. Sendo o delineamento em quadrados latinos um caso particular de modelos com três fatores cruzados, sem interação e sem caselas vazias, onde somente um dos fatores é de interesse (tratamentos) e os outros dois fatores (linhas e colunas) são apenas fatores de restrição na casualização, é possível testar hipóteses do tipo :

$$
\mathrm{H}_{0}: \mathrm{t}_{1}=\mathrm{t}_{2}=\ldots=\mathrm{t}_{\mathrm{K}} \text {, }
$$

mesmo no caso de número diferente de observações por unidade experimental, exceto para algumas somas de quadrados do tipo I, pois as hipóteses testadas dependem da ordenação dos fatores no modelo e, conseqüentemente, num eventual programa para análise estatística dos dados, como por exemplo o SAS. Nesse contexto, os efeitos de linhas e de colunas devem anteceder os de tratamentos, na configuração do modelo.

2. A soma de quadrados tipo I difere das demais pois, não há no modelo presença de interação e nem caselas vazias. Desta forma deve-se salientar 
que o pesquisador deve tomar cuidado com a interpretação de resultados de análise estatística, pois nem sempre as hipóteses testadas são as de igualdade de tratamentos, principalmente para as somas de quadrados do tipo I .

3. O quadrado latino com número diferente de observações por unidade experimental mostrou ser um delineamento robusto ao desbalanceamento, pois, os resultados da análise praticamente não se alteram para os quatro tipos de somas de quadrados.

4. Na prática, conhecendo os mecanismos das quatro somas de quadrados e construindo suas funções estimáveis, o pesquisador terá condições de optar e de realizar uma análise que seja mais adequada para o seu problema, tendo conhecimento da hipótese que está sendo testada. 


\section{REFERÊNCIAS BIBLIOGRÁFICAS}

ANDERSON, V. L. \& MCLEAN, R. A. Design of experiments. New York, Marcel Dekker, 1974. 418p.

BUENO, B. F. Análise da variância de ensaios em quadrado latino quando há perda de duas parcelas. Piracicaba, ESALQ, 1976. 20p.

CAMPOS, H. Estudo sobre a análise de experimentos com parcelas perdidas. Piracicaba, 1964. 84p. (Doutorado - Escola Superior de Agricultura "Luiz de Queiroz"/USP).

CAMPOS, H. \& PIEDADE, S. M. S. Planejamento de experimentos. Piracicaba, FEALQ, 1992. 185p.

CHAKRABARTI, M. C. Mathematics of design and analysis. London, Asia Publishing House, 1962. 121p.

COCHRAN, W. G. \& COX, G. M. Experimental designs. 2.ed. New York, John Wiley, 1957.617p. 
FEDERER, W.T. Experimental design : theory and application. New York, Macmillan, 1955. 520p.

FEDERER, W.T. Statistical design and analysis for intercropping experiments. New York, Springer Verlag, 1993. 298p.

GILL, J.L. Design and analysis of experiments in the animal and medical sciences. 2.ed. Iowa, Ames, The Iowa State University Press, 1981. 301p.

GOMES, F. P. Curso de estatística experimental. 8.ed. Piracicaba, Nobel, 1978. 430p.

GOMES, F. P. A estatística moderna na pesquisa agropecuária. 8.ed. Piracicaba, Assoc. Bras. de Pesquisa da Potassa e do Fosfato, 1984. $160 \mathrm{p}$.

GRAYBILL, F. A. An introduction to linear statistical models. New York, McGraw-Hill, 1961. v.1. 463p.

HEIBERGER, R. M. Computation for the analysis of designed experiments. New York, John Wiley, 1989. 683p. 
IEMMA, A. F. \& PALM, R. Les matrices inverses generalisées et utilisation dans le modele linéaire. Notes de Statistique et D'informatique, Faculté des Sciences Agronomiques, Gembloux, Bélgica, $\underline{92}$ (1) : 26 p., 1992.

IEMMA, A. F. Analisis de varianza com datos desbalanceados. Santa Fé de Bogota, Universidad Nacional de Colômbia / Deptọ. de Matemáticas y Estadísticas, 1993. 102p.

IEMMA, A. F. Análise de variância de dados desbalanceados. $4^{\circ}$ Congresso Nacional de Usuários do "SAS". Piracicaba, CIAGRI/USP, 1995-a. 111p.

IEMMA, A. F. Dados estatísticos desbalanceados : PROC SAS/GLM. Rio de Janeiro, UFRRJ / Instituto de Ciências Exatas, 1995-b. 130p.

IEMMA, A. F. Que hipóteses testamos através do SAS em presença de caselas vazias ? Piracicaba, Scientia Agricola, 52 (2) : 209 - 19, 1995-c.

JOHN, J. A. Cyclic designs. New York, Chapman and Hall, 1987. 232p.

JOHN, P. W. M. Statistical design and analysis of experiments. New York, Macmillan Company, 1971. 356p. 
KEMPTHORNE, $O$. The design and analysis of experiments. New York, Robert E. Krieger, 1975.631p.

KIRK, R. E. Experimental design : procedures for the behavioral sciences. Belmont, Brooks/Cole Publishing, 1968. 577p.

LITTLE, T. \& HILLS, J. Métodos estadísticos para la investigación en la agricultura. 2.ed. México, Ed. Trillás, 1975. 302p.

LITTLE, T. \& HILLS, J. Agricultural experimentation : design and analysis. New York, John Wiley, 1978. 350p.

MILLIKEN, G. A. \& JOHNSON, D. E. Analysis of messy data. New York, Van Nostrand Reinhold, 1984. v.1: Designed Experiments, 473p.

MONDARDO, M. Estimabilidade de funções paramétricas com dados desbalanceados através do PROC GLM do SAS : aplicações à pesquisa agropecuária. Piracicaba, 1994. 166p. (Mestrado - Escola Superior de Agricultura "Luiz de Queiroz"/USP).

MURRAY, L. W. Estimation of missing cells in randomized block and latin square designs. Cambridge. The American Statistician, $\underline{40}$ (4) : 289 93, 1986. 
NETER, J. \& WASSERMAN, W. Applied linear statistical models : regression analysis of variance and experimental designs. Illinois, Richard D. Irvin, 1974. 842p.

OGAWA, J. Statistical theory of the analysis of experimental designs. New York, Marcel Dekker, 1974. 465p.

OSTLE, B. Statistics in research : basic concepts and techniques for research workers. 2.ed. Ames, The Iowa State College Press, 1956. $487 p$.

PANSE, V. G. \& SUKHATME, P. V. Métodos estadísticos para investigadores agrícolas. 2.ed. México, Fondo de Cultura Económica, 1963. 349p.

PATERSON, D. D. Aplicação da estatística à experimentação agrícola. Rio de Janeiro, Ministério da Agricultura / Centro Nacional de Pesquisas Agronômicas, 1946. 86p.

PEARCE, S. C. Field experimentation with fruit trees and other perennial plants. Slagh, Comm. Agr. Bureaux, 1976. 182p.

RAGHAVARAO, D. Constructions and combinatorial problems in design of experiments. New York, John Wiley, 1971. 386p. 
RIBOLDI, J. A decomposição da soma de quadrados de tratamentos nos delineamentos em blocos incompletos parcialmente balanceados. Piracicaba, 1988. 165p. (Doutorado - Escola Superior de Agricultura "Luiz de Queiroz"/USP).

SANCHES, S. F. Análise estatística de um experimento em quadrado latino com perda de parcela, linha, coluna ou tratamento. Piracicaba, 1978. 100p. (Mestrado - Escola Superior de Agricultura "Luiz de Queiroz"/USP).

SANTOS, E. S. Utilização de "softwares" estatísticos na interpretação de hipóteses com dados desbalanceados. Piracicaba, 1994. 175p. (Doutorado - Escola Superior de Agricultura "Luiz de Queiroz"/USP).

SAS INSTITUTE. User's guide statistics version. 6.ed. Cary, 1990. 846p.

SCHEFFE, H. The analysis of variance. New York, John Wiley, 1959. $477 \mathrm{p}$.

SEARLE, S. R. Linear models for unbalanced data. New York, John Wiley, 1987. 536p.

SNEDECOR, G. W. \& COCHRAN, W. G. Statistical methods. 6.ed. Ames, lowa, State University Press, 1967. 593p. 
STREET, A. P. \& STREET, D. J. Combinatorics of experimental design. Oxford, Oxford University Press, 1987. 400p.

VAJDA, S. The mathematics of experimental designs: incomplete block designs and latin squares. New York, Hafner Publishing, 1967. 110p.

WINNER, B. J. Statistical principles in experimental design. 2ed. New York, McGraw-Hill, 1971. 907p.

WISHART, J. \& SANDERS, H. G. Princípios e prática da experimentação de campo. London, The Empire Cotton Growing Corporation, 1936. 108p. 
108.

A P ÊN DICE 
Programa SAS utilizado para o exemplo ilustrativo

DATA TESE;

OPTIONS NODATE;

INPUT $1 \mathrm{c}$ t rep dados;

CARDS;

11111.64

12210.62

12220.61

13310.41

13320.39

13330.37

13340.38

21311.11

21321.09

21331.10

21341.12

21351.11

22110.81

22120.80

22130.82

23210.62

23220.62

23230.61

31212.13

$\begin{array}{llll}3 & 1222.11\end{array}$

32311.86

32321.88

32331.85

32341.84

32351.84

32361.80

33111.43

;

PROC PRINT; RUN;

PROC GLM;

CLASS $1 \mathrm{c} t$;

MODEL dados=1 c t/SOLUTION E E1 E2 E3 E4 SS1 SS2 SS3 SS4;

RUN; 
Salienta-se que no programa anterior, a ordem de entrada dos efeitos de fatores no modelo, ou seja no comando MODEL, foi linha, coluna, tratamento.

Para as outras ordenações, o modelo é semelhante, diferindo apenas na ordem dos fatores no comando MODEL . 
Resultados do SAS para o quadrado latino com número diferente de observações por unidade experimental, com todas as caselas ocupadas.

1. Ordenação : Linha, Coluna, Tratamento

The SAS System

OBS $\quad \mathrm{L} \quad \mathrm{C} \quad \mathrm{T}$ REP DADOS

$\begin{array}{llllll}1 & 1 & 1 & 1 & 1 & 1.64\end{array}$

$\begin{array}{llllll}2 & 1 & 2 & 2 & 1 & 0.62\end{array}$

$\begin{array}{llllll}3 & 1 & 2 & 2 & 2 & 0.61\end{array}$

$\begin{array}{llllll}4 & 1 & 3 & 3 & 1 & 0.41\end{array}$

$\begin{array}{llllll}5 & 1 & 3 & 3 & 2 & 0.39\end{array}$

$\begin{array}{llllll}6 & 1 & 3 & 3 & 3 & 0.37\end{array}$

$\begin{array}{llllll}7 & 1 & 3 & 3 & 4 & 0.38\end{array}$

$\begin{array}{llllll}8 & 2 & 1 & 3 & 1 & 1.11\end{array}$

$\begin{array}{llllll}9 & 2 & 1 & 3 & 2 & 1.09\end{array}$

$\begin{array}{llllll}10 & 2 & 1 & 3 & 3 & 1.10\end{array}$

$\begin{array}{llllll}11 & 2 & 1 & 3 & 4 & 1.12\end{array}$

$\begin{array}{llllll}12 & 2 & 1 & 3 & 5 & 1.11\end{array}$

$\begin{array}{llllll}13 & 2 & 2 & 1 & 1 & 0.81\end{array}$

$\begin{array}{llllll}14 & 2 & 2 & 1 & 2 & 0.80\end{array}$

$\begin{array}{llllll}15 & 2 & 2 & 1 & 3 & 0.82\end{array}$

$\begin{array}{llllll}16 & 2 & 3 & 2 & 1 & 0.62\end{array}$

$\begin{array}{llllll}17 & 2 & 3 & 2 & 2 & 0.62\end{array}$

$\begin{array}{llllll}18 & 2 & 3 & 2 & 3 & 0.61\end{array}$

$\begin{array}{llllll}19 & 3 & 1 & 2 & 1 & 2.13\end{array}$

$\begin{array}{llllll}20 & 3 & 1 & 2 & 2 & 2.11\end{array}$

$\begin{array}{llllll}21 & 3 & 2 & 3 & 1 & 1.86\end{array}$

$\begin{array}{llllll}22 & 3 & 2 & 3 & 2 & 1.88\end{array}$

$\begin{array}{llllll}23 & 3 & 2 & 3 & 3 & 1.85\end{array}$

$\begin{array}{llllll}24 & 3 & 2 & 3 & 4 & 1.84\end{array}$

$\begin{array}{llllll}25 & 3 & 2 & 3 & 5 & 1.84\end{array}$

$\begin{array}{llllll}26 & 3 & 2 & 3 & 6 & 1.80\end{array}$

$\begin{array}{llllll}27 & 3 & 3 & 1 & 1 & 1.43\end{array}$ 
General Linear Models Procedure

Class Level Information

Class Levels Values

L $\quad 3 \quad 123$

C $\quad 3 \quad 123$

$\begin{array}{lll}\text { T } & 3 & 123\end{array}$

Number of observations in data set $=27$

General Form of Estimable Functions

Effect Coefficients

\section{INTERCEPT L1}

L $\quad 1 \quad$ L2

$2 \quad \mathrm{~L} 3$

3 L1-L2-L3

C 11 L5

$2 \quad$ L6

3 L1-L5-L6

T $1 \quad$ L8

$2 \quad$ L9

3 L1-L8-L9 
Type I Estimable Functions for: L

\section{Effect Coefficients}

\section{INTERCEPT 0}

$\begin{array}{lll}\text { L } & 1 & \text { L2 } \\ & 2 & \text { L3 } \\ & 3 & -\mathrm{L} 2-\mathrm{L} 3 \\ & & \\ \mathrm{C} & 1 & -0.0794^{* \mathrm{~L} 2} 2+0.2323 * \mathrm{~L} 3 \\ & 2 & -0.381 * \mathrm{~L} 2-0.3939 * \mathrm{~L} 3 \\ & 3 & 0.4603 * \mathrm{~L} 2+0.1616^{*} \mathrm{~L} 3\end{array}$

$\begin{array}{lll}\mathrm{T} & 1 & 0.0317^{*} \mathrm{~L} 2+0.1616^{*} \mathrm{~L} 3 \\ & 2 & 0.0635^{*} \mathrm{~L} 2+0.0505^{*} \mathrm{~L} 3 \\ & 3 & -0.0952 * \mathrm{~L} 2-0.2121 * \mathrm{~L} 3\end{array}$

Type I Estimable Functions for: C

Effect Coefficients

\section{INTERCEPT 0}

$\begin{array}{lll}\text { L } & 1 & 0 \\ & 2 & 0 \\ & 3 & 0 \\ & & \\ \text { C } & 1 & \text { L5 } \\ & 2 & \text { L6 } \\ & 3 & \text {-L5-L6 }\end{array}$

$\begin{array}{lll}\text { T } & 1 & -0.0211 * \text { L5 } 5+0.2079 * \text { L6 } \\ & 2 & -0.1345 * \text { L5-0.2004*L6 } \\ & 3 & 0.1557 * \text { L5-0.0075*L6 }\end{array}$ 
Type I Estimable Functions for: T

\section{Effect Coefficients}

\section{INTERCEPT 0}

$\begin{array}{lll}\mathrm{L} & 1 & 0 \\ & 2 & 0 \\ & 3 & 0\end{array}$

$\begin{array}{lll}\text { C } & 1 & 0 \\ & 2 & 0 \\ & 3 & 0\end{array}$

$\begin{array}{lll}\text { T } & 1 & \text { L8 } \\ & 2 & \text { L9 } \\ & 3 & \text {-L8-L9 }\end{array}$

Type II Estimable Functions for: L

\section{Effect Coefficients}

\section{INTERCEPT 0}

L $\quad 1 \quad$ L2

$2 \quad \mathrm{~L} 3$

$3 \quad-\mathrm{L} 2-\mathrm{L} 3$

C 110

$\begin{array}{ll}2 & 0 \\ 3 & 0\end{array}$

$\begin{array}{lll}\mathrm{T} & 1 & 0\end{array}$

20

30 
Type II Estimable Functions for: C

Effect Coefficients

INTERCEPT 0

L $\quad 1 \quad 0$

20

30

C $\quad 1 \quad$ L5

$2 \quad \mathrm{~L} 6$

3 -L5-L6

$\begin{array}{lll}\text { T } & 1 & 0 \\ & 2 & 0\end{array}$

30

Type II Estimable Functions for: $T$

\section{Effect Coefficients}

\section{INTERCEPT 0}

$\begin{array}{lll}\mathrm{L} & 1 & 0 \\ & 2 & 0 \\ & 3 & 0\end{array}$

C 110

$\begin{array}{ll}2 & 0 \\ 3 & 0\end{array}$

$\begin{array}{lll}\text { T } & 1 & \text { L8 } \\ & 2 & \text { L9 } \\ & 3 & \text {-L8-L9 }\end{array}$


Type III Estimable Functions for: $\mathbf{L}$

Effect Coefficients

INTERCEPT 0

L $1 \quad$ L2

$2 \quad \mathrm{~L} 3$

$3 \quad-\mathrm{L} 2-\mathrm{L} 3$

$\begin{array}{lll}\mathrm{C} & 1 & 0\end{array}$

20

30

$\begin{array}{lll}\text { T } & 1 & 0 \\ & 2 & 0\end{array}$

30

Type III Estimable Functions for: C

Effect Coefficients

INTERCEPT 0

$\begin{array}{lll}\mathrm{L} & 1 & 0 \\ & 2 & 0 \\ & 3 & 0\end{array}$

C 11 L5

$2 \quad$ L6

3 -L5-L6

$\begin{array}{lll}\text { T } & 1 & 0 \\ & 2 & 0 \\ & 3 & 0\end{array}$


Type III Estimable Functions for: T

Effect Coefficients

INTERCEPT 0

$\begin{array}{lll}\text { L } & 1 & 0 \\ & 2 & 0 \\ & 3 & 0\end{array}$

C $\quad 1 \quad 0$

$\begin{array}{ll}2 & 0 \\ 3 & 0\end{array}$

$\begin{array}{lll}\mathrm{T} & 1 & \mathrm{~L} 8\end{array}$

$3 \quad-L 8-L 9$

Type IV Estimable Functions for: L

\section{Effect Coefficients}

\section{INTERCEPT 0}

L $\quad 1 \quad$ L2

$2 \mathrm{~L} 3$

3 -L2-L3

C $\quad 1 \quad 0$

20

30

$\begin{array}{lll}\text { T } & 1 & 0 \\ & 2 & 0 \\ & 3 & 0\end{array}$


Type IV Estimable Functions for: C

Effect Coefficients

INTERCEPT 0

L $\quad 1 \quad 0$

20

30

C 11 L5

$2 \quad$ L6

$3 \quad-\mathrm{L} 5-\mathrm{L} 6$

$\begin{array}{lll}\text { T } & 1 & 0 \\ & 2 & 0 \\ & 3 & 0\end{array}$

Type IV Estimable Functions for: T

Effect Coefficients

INTERCEPT 0

$\begin{array}{lll}\mathrm{L} & 1 & 0 \\ & 2 & 0 \\ & 3 & 0\end{array}$

C 110

20

30

$\begin{array}{lll}\mathrm{T} & 1 & \mathrm{~L} 8 \\ & 2 & \end{array}$

$2 \quad$ L9

3 -L8-L9 
Dependent Variable: DADOS

\begin{tabular}{|c|c|c|c|c|c|}
\hline & \multicolumn{2}{|c|}{ Sum of } & \multicolumn{3}{|l|}{ Mean } \\
\hline Source & DF & Squares & Square F & alue & $\operatorname{Pr}>F$ \\
\hline Model & 6 & 8.86271782 & 1.47711964 & 84.69 & 0.0001 \\
\hline ror & 20 & 0.34884514 & 0.01744226 & & \\
\hline
\end{tabular}

Corrected Total $\quad 26 \quad 9.21156296$

$\begin{array}{rrrc}\text { R-Square } & \text { C.V. } & \text { Root MSE } & \text { DADOS Mean } \\ 0.962130 & 11.51394 & 0.13207 & 1.14704\end{array}$

Source $\quad$ DF Type I SS Mean Square F Value $\operatorname{Pr}>$ F

$\begin{array}{llllrl}\mathrm{L} & 2 & 7.15231361 & 3.57615681 & 205.03 & 0.0001 \\ \mathrm{C} & 2 & 1.64972905 & 0.82486453 & 47.29 & 0.0001 \\ \mathrm{~T} & 2 & 0.06067515 & 0.03033758 & 1.74 & 0.2012\end{array}$

Source DF Type II SS Mean Square F Value $\operatorname{Pr}>$ F

$\begin{array}{llllrl}\mathrm{L} & 2 & 5.38851770 & 2.69425885 & 154.47 & 0.0001 \\ \mathrm{C} & 2 & 1.67134249 & 0.83567125 & 47.91 & 0.0001 \\ \mathrm{~T} & 2 & 0.06067515 & 0.03033758 & 1.74 & 0.2012\end{array}$

Source DF Type III SS Mean Square F Value $\operatorname{Pr}>$ F

$\begin{array}{llllrl}\mathrm{L} & 2 & 5.38851770 & 2.69425885 & 154.47 & 0.0001 \\ \mathrm{C} & 2 & 1.67134249 & 0.83567125 & 47.91 & 0.0001 \\ \mathrm{~T} & 2 & 0.06067515 & 0.03033758 & 1.74 & 0.2012\end{array}$

Source DF Type IV SS Mean Square F Value $\operatorname{Pr}>$ F

$\begin{array}{llllrl}\mathrm{L} & 2 & 5.38851770 & 2.69425885 & 154.47 & 0.0001 \\ \mathrm{C} & 2 & 1.67134249 & 0.83567125 & 47.91 & 0.0001 \\ \mathrm{~T} & 2 & 0.06067515 & 0.03033758 & 1.74 & 0.2012\end{array}$


T for H0: $\quad \operatorname{Pr}>|\mathrm{T}| \quad$ Std Error of

Parameter Estimate Parameter $=0 \quad$ Estimate

\begin{tabular}{lccccc}
\multicolumn{2}{l}{ INTERCEPT } & $1.509559352 \mathrm{~B}$ & 20.37 & 0.0001 & 0.07411793 \\
$\mathrm{~L}$ & 1 & $-1.077045501 \mathrm{~B}$ & -14.75 & 0.0001 & 0.07299579 \\
& 2 & $-1.042648251 \mathrm{~B}$ & -15.85 & 0.0001 & 0.06579476 \\
& 3 & $0.000000000 \mathrm{~B}$ &. &. & \\
$\mathrm{C}$ & 1 & $0.681625372 \mathrm{~B}$ & 9.65 & 0.0001 & 0.07060313 \\
& 2 & $0.269984354 \mathrm{~B}$ & 3.91 & 0.0009 & 0.06905915 \\
& 3 & $0.000000000 \mathrm{~B}$ &. &. & \\
$\mathrm{~T}$ & 1 & $0.133123012 \mathrm{~B}$ & 1.85 & 0.0787 & 0.07183746 \\
& 1 & $0.018842977 \mathrm{~B}$ & 0.31 & 0.7620 & 0.06138356
\end{tabular}

NOTE: The X'X matrix has been found to be singular and a generalized inverse was used to solve the normal equations. Estimates followed by the letter ' $\mathrm{B}$ ' are biased, and are not unique estimators of the parameters.

2. Ordenação : Linha, Tratamento, Coluna 


\section{The SAS System}

\section{OBS $\quad \mathrm{L} \quad \mathrm{C} \quad \mathrm{T}$ REP DADOS}

$\begin{array}{cccccc}1 & 1 & 1 & 1 & 1 & 1.64 \\ 2 & 1 & 2 & 2 & 1 & 0.62 \\ 3 & 1 & 2 & 2 & 2 & 0.61 \\ 4 & 1 & 3 & 3 & 1 & 0.41 \\ 5 & 1 & 3 & 3 & 2 & 0.39 \\ 6 & 1 & 3 & 3 & 3 & 0.37 \\ 7 & 1 & 3 & 3 & 4 & 0.38 \\ 8 & 2 & 1 & 3 & 1 & 1.11 \\ 9 & 2 & 1 & 3 & 2 & 1.09 \\ 10 & 2 & 1 & 3 & 3 & 1.10 \\ 11 & 2 & 1 & 3 & 4 & 1.12 \\ 12 & 2 & 1 & 3 & 5 & 1.11 \\ 13 & 2 & 2 & 1 & 1 & 0.81 \\ 14 & 2 & 2 & 1 & 2 & 0.80 \\ 15 & 2 & 2 & 1 & 3 & 0.82 \\ 16 & 2 & 3 & 2 & 1 & 0.62 \\ 17 & 2 & 3 & 2 & 2 & 0.62 \\ 18 & 2 & 3 & 2 & 3 & 0.61 \\ 19 & 3 & 1 & 2 & 1 & 2.13 \\ 20 & 3 & 1 & 2 & 2 & 2.11 \\ 21 & 3 & 2 & 3 & 1 & 1.86 \\ 22 & 3 & 2 & 3 & 2 & 1.88 \\ 23 & 3 & 2 & 3 & 3 & 1.85 \\ 24 & 3 & 2 & 3 & 4 & 1.84 \\ 25 & 3 & 2 & 3 & 5 & 1.84 \\ 26 & 3 & 2 & 3 & 6 & 1.80 \\ 27 & 3 & 3 & 1 & 1 & 1.43\end{array}$




\section{General Linear Models Procedure}

Class Level Information

Class Levels Values

L $\quad 3 \quad 123$

C $\quad 3 \quad 123$

$\begin{array}{lll}\mathrm{T} & 3 \quad 123\end{array}$

Number of observations in data set $=27$

General Form of Estimable Functions

Effect Coefficients

INTERCEPT LI

L $\quad 1 \quad$ L2

$2 \quad \mathrm{~L} 3$

$3 \quad$ L1-L2-L3

$\begin{array}{lll}\text { T } & 1 & \text { L5 }\end{array}$

$2 \quad$ L6

3 L1-L5-L6

$\begin{array}{lll}\mathrm{C} & 1 & \mathrm{~L} 8\end{array}$

$2 \quad$ L9

3 L1-L8-L9 
Type I Estimable Functions for: $\mathrm{L}$

Effect Coefficients

INTERCEPT 0

L $11 \quad$ L2

$2 \quad$ L3

3 -L2-L3

$\begin{array}{ccc}\mathrm{T} & 1 & 0.0317^{*} \mathrm{~L} 2+0.1616^{*} \mathrm{~L} 3 \\ & 2 & 0.0635^{*} \mathrm{~L} 2+0.0505^{*} \mathrm{~L} 3 \\ & 3 & -0.0952 * \mathrm{~L} 2-0.2121 * \mathrm{~L} 3\end{array}$

C $\quad 1 \quad-0.0794 * \mathrm{~L} 2+0.2323 * \mathrm{~L} 3$

$2 \quad-0.381 * \mathrm{~L} 2-0.3939 * \mathrm{~L} 3$

$3 \quad 0.4603 * \mathrm{~L} 2+0.1616 * \mathrm{~L} 3$

Type I Estimable Functions for: T

Effect Coefficients

INTERCEPT 0

$\begin{array}{lll}\text { L } & 1 & 0 \\ & 2 & 0 \\ & 3 & 0\end{array}$

T $1 \quad$ L5

$2 \quad$ L6

3 -L5-L6

C $11-0.2098 *$ L5-0.072*L6

$2 \quad 0.291 *$ L5-0.0652*L6

$3-0.0811 * \mathrm{~L} 5+0.1373 * \mathrm{~L} 6$ 
Type I Estimable Functions for: C

\section{Effect Coefficients}

\section{INTERCEPT 0}

$\begin{array}{lll}\mathrm{L} & 1 & 0 \\ & 2 & 0 \\ & 3 & 0\end{array}$

$\begin{array}{lll}\text { T } & 1 & 0 \\ & 2 & 0 \\ & 3 & 0\end{array}$

$\begin{array}{lll}\text { C } & 1 & \text { L8 } \\ & 2 & \text { L9 } \\ & 3 & \text {-L8-L9 }\end{array}$

Type II Estimable Functions for: L

Effect Coefficients

\section{INTERCEPT 0}

$\begin{array}{ccc}\text { L } & 1 & \text { L2 } \\ & 2 & \text { L3 } \\ & 3 & \text {-L2 } \\ & & \\ \mathrm{T} & 1 & 0 \\ & 2 & 0 \\ & 3 & 0 \\ & & \\ \mathrm{C} & 1 & 0 \\ & 2 & 0 \\ & 3 & 0\end{array}$


Type II Estimable Functions for: T

\section{Effect Coefficients}

\section{INTERCEPT 0}

$\begin{array}{lll}\mathrm{L} & 1 & 0 \\ & 2 & 0 \\ & 3 & 0\end{array}$

$\begin{array}{lll}\text { T } & 1 & \text { L5 } \\ & 2 & \text { L6 } \\ & 3 & \\ & & \text {-L5 } \\ \text { C } & 1 & 0 \\ & 2 & 0 \\ & 3 & 0\end{array}$

Type II Estimable Functions for: C

\section{Effect Coefficients}

\section{INTERCEPT 0}

$\begin{array}{lll}\mathrm{L} & 1 & 0 \\ & 2 & 0 \\ & 3 & 0\end{array}$

$\begin{array}{lll}\text { T } & 1 & 0 \\ & 2 & 0\end{array}$

C 11 L8

2 L9

3 -L8-L9 
Type III Estimable Functions for: L

Effect Coefficients

\section{INTERCEPT 0}

$\begin{array}{lll}\text { L } & 1 & \text { L2 } \\ & 2 & \text { L3 } \\ & 3 & \text {-L2-L3 }\end{array}$

$\begin{array}{lll}\text { T } & 1 & 0 \\ & 2 & 0 \\ & 3 & 0\end{array}$

$\begin{array}{lll}\mathrm{C} & 1 & 0 \\ & 2 & 0 \\ & 3 & 0\end{array}$

Type III Estimable Functions for: T

\section{Effect Coefficients}

\section{INTERCEPT 0}

L $\quad 1 \quad 0$

20

30

T $1 \quad$ L5

$2 \quad$ L6

3 -L5-L6

C $\quad 1 \quad 0$

20

30 
Type III Estimable Functions for: C

\section{Effect Coefficients}

INTERCEPT 0

$\begin{array}{lll}\mathrm{L} & 1 & 0 \\ & 2 & 0 \\ & 3 & 0\end{array}$

$\begin{array}{ccc}\text { T } & 1 & 0 \\ & 2 & 0 \\ & 3 & 0\end{array}$

$\begin{array}{lll}\text { C } & 1 & \text { L8 } \\ & 2 & \text { L9 } \\ & 3 & \text {-L8-L9 }\end{array}$

Type IV Estimable Functions for: L

\section{Effect Coefficients}

\section{INTERCEPT 0}

$\begin{array}{lll}\mathrm{L} & 1 & \mathrm{~L} 2 \\ & 2 & \mathrm{~L} 3 \\ & 3 & \text {-L2-L3 }\end{array}$

$\begin{array}{lll}\text { T } & 1 & 0 \\ & 2 & 0 \\ & 3 & 0\end{array}$

$\begin{array}{ccc}\text { C } & 1 & 0 \\ & 2 & 0 \\ & 3 & 0\end{array}$


Type IV Estimable Functions for: T

\section{Effect Coefficients}

\section{INTERCEPT 0}

$\begin{array}{lll}\mathrm{L} & 1 & 0 \\ & 2 & 0 \\ & 3 & 0\end{array}$

T $1 \quad$ L5

$2 \quad$ L6

3 -L5-L6

$\begin{array}{lll}\mathrm{C} & 1 & 0 \\ & 2 & 0 \\ & 3 & 0\end{array}$

Type IV Estimable Functions for: C

\section{Effect Coefficients}

\section{INTERCEPT 0}

$\begin{array}{lll}\mathrm{L} & 1 & 0 \\ & 2 & 0 \\ & 3 & 0\end{array}$

$\begin{array}{lll}\text { T } & 1 & 0 \\ & 2 & 0 \\ & 3 & 0\end{array}$

$\begin{array}{lll}\text { C } & 1 & \text { L8 } \\ & 2 & \text { L9 } \\ & 3 & \text {-L8-L9 }\end{array}$


Dependent Variable: DADOS

\begin{tabular}{lccccc} 
& \multicolumn{2}{c}{ Sum of } & \multicolumn{2}{c}{ Mean } \\
Source & DF & Squares & Square F Value & $\operatorname{Pr}>$ F \\
Model & 6 & 8.86271782 & 1.47711964 & 84.69 & 0.0001 \\
& 20 & 0.34884514 & 0.01744226 & & \\
Error & & &
\end{tabular}

Corrected Total $\quad 26 \quad 9.21156296$

$\begin{array}{rrrc}\text { R-Square } & \text { C.V. } & \text { Root MSE } & \text { DADOS Mean } \\ 0.962130 & 11.51394 & 0.13207 & 1.14704\end{array}$

Source DF Type I SS Mean Square F Value $\operatorname{Pr}>$ F

$\begin{array}{rrrrrr}\mathrm{L} & 2 & 7.15231361 & 3.57615681 & 205.03 & 0.0001 \\ \mathrm{~T} & 2 & 0.03906172 & 0.01953086 & 1.12 & 0.3460 \\ \mathrm{C} & 2 & 1.67134249 & 0.83567125 & 47.91 & 0.0001\end{array}$

Source DF Type II SS Mean Square F Value $\operatorname{Pr}>$ F

$\begin{array}{llllrl}\mathrm{L} & 2 & 5.38851770 & 2.69425885 & 154.47 & 0.0001 \\ \mathrm{~T} & 2 & 0.06067515 & 0.03033758 & 1.74 & 0.2012 \\ \mathrm{C} & 2 & 1.67134249 & 0.83567125 & 47.91 & 0.0001\end{array}$

Source DF Type III SS Mean Square F Value $\operatorname{Pr}>$ F

$\begin{array}{llllrl}\mathrm{L} & 2 & 5.38851770 & 2.69425885 & 154.47 & 0.0001 \\ \mathrm{~T} & 2 & 0.06067515 & 0.03033758 & 1.74 & 0.2012 \\ \mathrm{C} & 2 & 1.67134249 & 0.83567125 & 47.91 & 0.0001\end{array}$

Source DF Type IV SS Mean Square F Value $\operatorname{Pr}>$ F

$\begin{array}{llllrl}\mathrm{L} & 2 & 5.38851770 & 2.69425885 & 154.47 & 0.0001 \\ \mathrm{~T} & 2 & 0.06067515 & 0.03033758 & 1.74 & 0.2012 \\ \mathrm{C} & 2 & 1.67134249 & 0.83567125 & 47.91 & 0.0001\end{array}$




\begin{tabular}{|c|c|c|c|c|c|}
\hline \multirow{3}{*}{\multicolumn{2}{|c|}{$\begin{array}{l}\text { Parameter } \\
\text { INTERCEPT }\end{array}$}} & \multicolumn{4}{|c|}{$\mathrm{T}$ for $\mathrm{H} 0: \quad \operatorname{Pr}>|\mathrm{T}| \quad$ Std Error of } \\
\hline & & \multicolumn{2}{|c|}{ Estimate $\quad$ Parameter $=0$} & \multicolumn{2}{|c|}{ Estimate } \\
\hline & & $1.509559352 \mathrm{~B}$ & 20.37 & 0.0001 & 0.07411793 \\
\hline \multirow[t]{3}{*}{ L } & 1 & $-1.077045501 \mathrm{~B}$ & -14.75 & 0.0001 & 0.07299579 \\
\hline & 2 & $-1.042648251 B$ & -15.85 & 0.0001 & 0.06579476 \\
\hline & 3 & $0.000000000 \mathrm{~B}$ & & & \\
\hline \multirow[t]{3}{*}{$\Gamma$} & 1 & $0.133123012 \mathrm{~B}$ & 1.85 & 0.0787 & $0.0^{\prime}$ \\
\hline & 2 & $0.018842977 \mathrm{~B}$ & 0.31 & 0.7620 & 0.06138356 \\
\hline & 3 & $0.000000000 \mathrm{~B}$ & & & \\
\hline & 1 & $0.681625372 \mathrm{~B}$ & 9.65 & 0.00 & 0.070 \\
\hline & 2 & $0.269984354 \mathrm{~B}$ & 3.91 & 0.0009 & 0.06905915 \\
\hline & 3 & $0.000000000 \mathrm{~B}$ & & & \\
\hline
\end{tabular}

NOTE: The $\mathrm{X}$ 'X matrix has been found to be singular and a generalized inverse was used to solve the normal equations. Estimates followed by the letter ' $\mathrm{B}$ ' are biased, and are not unique estimators of the parameters. 
3. Ordenação : Coluna, Tratamento, Linha

The SAS System

\section{OBS $\quad \mathrm{L} \quad \mathrm{C} \quad \mathrm{T}$ REP DADOS}

$\begin{array}{cccccc}1 & 1 & 1 & 1 & 1 & 1.64 \\ 2 & 1 & 2 & 2 & 1 & 0.62 \\ 3 & 1 & 2 & 2 & 2 & 0.61 \\ 4 & 1 & 3 & 3 & 1 & 0.41 \\ 5 & 1 & 3 & 3 & 2 & 0.39 \\ 6 & 1 & 3 & 3 & 3 & 0.37 \\ 7 & 1 & 3 & 3 & 4 & 0.38 \\ 8 & 2 & 1 & 3 & 1 & 1.11 \\ 9 & 2 & 1 & 3 & 2 & 1.09 \\ 10 & 2 & 1 & 3 & 3 & 1.10 \\ 11 & 2 & 1 & 3 & 4 & 1.12 \\ 12 & 2 & 1 & 3 & 5 & 1.11 \\ 13 & 2 & 2 & 1 & 1 & 0.81 \\ 14 & 2 & 2 & 1 & 2 & 0.80 \\ 15 & 2 & 2 & 1 & 3 & 0.82 \\ 16 & 2 & 3 & 2 & 1 & 0.62 \\ 17 & 2 & 3 & 2 & 2 & 0.62 \\ 18 & 2 & 3 & 2 & 3 & 0.61 \\ 19 & 3 & 1 & 2 & 1 & 2.13 \\ 20 & 3 & 1 & 2 & 2 & 2.11 \\ 21 & 3 & 2 & 3 & 1 & 1.86 \\ 22 & 3 & 2 & 3 & 2 & 1.88 \\ 23 & 3 & 2 & 3 & 3 & 1.85 \\ 24 & 3 & 2 & 3 & 4 & 1.84 \\ 25 & 3 & 2 & 3 & 5 & 1.84 \\ 26 & 3 & 2 & 3 & 6 & 1.80 \\ 27 & 3 & 3 & 1 & 1 & 1.43\end{array}$


General Linear Models Procedure

Class Level Information

Class Levels Values

L $\quad 3 \quad 123$

C $\quad 3 \quad 123$

$\begin{array}{lll}\mathrm{T} & 3 & 123\end{array}$

Number of observations in data set $=27$

General Form of Estimable Functions

Effect Coefficients

INTERCEPT L1

C 11 L2

$2 \quad$ L3

3 L1-L2-L3

T 11 L5

$2 \quad$ L6

3 L1-L5-L6

$\begin{array}{lll}\text { L } & 1 & \text { L8 }\end{array}$

$2 \quad$ L9

3 L1-L8-L9 
Type I Estimable Functions for: C

Effect Coefficients

\begin{tabular}{lll}
\multicolumn{2}{l}{ INTERCEPT } & 0 \\
& & \\
$\mathrm{C}$ & 1 & $\mathrm{~L} 2$ \\
& 2 & $\mathrm{~L} 3$ \\
& 3 & $-\mathrm{L} 2-\mathrm{L} 3$ \\
& & \\
$\mathrm{~T}$ & 1 & $0.1477 * \mathrm{~L} 3$ \\
& 2 & $-0.125 * \mathrm{~L} 2-0.1932 * \mathrm{~L} 3$ \\
& 3 & $0.125 * \mathrm{~L} 2+0.0455 * \mathrm{~L} 3$ \\
& & \\
$\mathrm{~L}$ & 1 & $-0.375 * \mathrm{~L} 2-0.3182 * \mathrm{~L} 3$ \\
& 2 & $0.25 * \mathrm{~L} 2-0.1023 * \mathrm{~L} 3$ \\
& 3 & $0.125 * \mathrm{~L} 2+0.4205 * \mathrm{~L} 3$
\end{tabular}

Type I Estimable Functions for: T

Effect Coefficients

INTERCEPT 0

$\begin{array}{lll}\mathrm{C} & 1 & 0 \\ & 2 & 0 \\ & 3 & 0\end{array}$

T 11 L5

$2 \quad$ L6

3 -L5-L6

L $\quad 1 \quad-0.0538 *$ L $5-0.0353 *$ L6

$2 \quad 0.3281 * \mathrm{~L} 5+0.0925 * \mathrm{~L} 6$

$3-0.2743 * \mathrm{~L} 5-0.0573 * \mathrm{~L} 6$ 
Type I Estimable Functions for: $\mathrm{L}$

\section{Effect Coefficients}

\begin{tabular}{lll}
\multicolumn{2}{l}{ INTERCEPT } & 0 \\
C & 1 & 0 \\
& 2 & 0 \\
& 3 & 0 \\
& & \\
$\mathrm{~T}$ & 1 & 0 \\
& 2 & 0 \\
& 3 & 0 \\
& & \\
L & 1 & L8 \\
& 2 & L9 \\
& 3 & -L8-L9
\end{tabular}

Type II Estimable Functions for: C

Effect Coefficients

\section{INTERCEPT 0}

$\begin{array}{lll}\text { C } & 1 & \text { L2 } \\ & 2 & \text { L3 } \\ & 3 & \text {-L2-L3 }\end{array}$

$\begin{array}{lll}\text { T } & 1 & 0 \\ & 2 & 0 \\ & 3 & 0\end{array}$

L $\quad 1 \quad 0$

20

30


Type II Estimable Functions for: T

\section{Effect Coefficients}

\section{INTERCEPT 0}

$\begin{array}{lll}\mathrm{C} & 1 & 0 \\ & 2 & 0 \\ & 3 & 0\end{array}$

$\begin{array}{lll}\text { T } & 1 & \text { L5 } \\ & 2 & \text { L6 } \\ & 3 & \text {-L5-L6 }\end{array}$

L $\quad 1 \quad 0$

20

30

Type II Estimable Functions for: L

\section{Effect Coefficients}

\section{INTERCEPT 0}

$\begin{array}{ccc}\text { C } & 1 & 0 \\ & 2 & 0 \\ & 3 & 0\end{array}$

$\begin{array}{lll}\text { T } & 1 & 0 \\ & 2 & 0 \\ & 3 & 0\end{array}$

$\begin{array}{lll}\text { L } & 1 & \text { L8 } \\ & 2 & \text { L9 } \\ & 3 & \text {-L8-L9 }\end{array}$


Type III Estimable Functions for: $\mathrm{C}$

\section{Effect Coefficients}

INTERCEPT
$\begin{array}{lll}\text { C } & 1 & \text { L2 } \\ & 2 & \text { L }: \\ & 3 & \text {-L2-L3 }\end{array}$

$\begin{array}{ccc}\text { T } & 1 & 0 \\ & 2 & 0 \\ & 3 & 0\end{array}$

$\begin{array}{lll}\text { L } & 1 & 0 \\ & 2 & 0 \\ & 3 & 0\end{array}$

Type III Estimable Functions for: T

Effect Coefficients

INTERCEPT 0

$\begin{array}{ccc}\text { C } & 1 & 0 \\ & 2 & 0 \\ & 3 & 0\end{array}$

T 11 L5

$2 \quad$ L6

$3 \quad-\mathrm{L} 5-\mathrm{L} 6$

L $\quad 1 \quad 0$

$\begin{array}{ll}2 & 0 \\ 3 & 0\end{array}$ 
Type III Estimable Functions for: L

\section{Effect Coefficients}

\begin{tabular}{lll}
\multicolumn{2}{c}{ INTERCEPT } & 0 \\
C & 1 & 0 \\
& 2 & 0 \\
& 3 & 0 \\
& & \\
T & 1 & 0 \\
& 2 & 0 \\
& 3 & 0 \\
& & \\
L & 1 & L8 \\
& 2 & L9 \\
& 3 & -L8-L9
\end{tabular}

Type IV Estimable Functions for: C

Effect Coefficients

\section{INTERCEPT 0}

$\begin{array}{lll}\text { C } & 1 & \text { L2 } \\ & 2 & \text { L3 } \\ & 3 & \text {-L2-L3 }\end{array}$

$\begin{array}{lll}\text { T } & 1 & 0 \\ & 2 & 0 \\ & 3 & 0\end{array}$

$\begin{array}{lll}\text { L } & 1 & 0\end{array}$

$\begin{array}{ll}2 & 0 \\ 3 & 0\end{array}$


Type IV Estimable Functions for: T

Effect Coefficients

INTERCEPT 0

$\begin{array}{lll}\mathrm{C} & 1 & 0 \\ & 2 & 0 \\ & 3 & 0\end{array}$

T 1 L5

2 L6

3 -L5-L6

$\begin{array}{lll}\mathbf{L} & 1 & 0 \\ & 2 & 0 \\ & 3 & 0\end{array}$

Type IV Estimable Functions for: L

Effect Coefficients

INTERCEPT 0

$\begin{array}{ccc}\text { C } & 1 & 0 \\ & 2 & 0 \\ & 3 & 0\end{array}$

$\begin{array}{lll}\text { T } & 1 & 0 \\ & 2 & 0 \\ & 3 & 0\end{array}$

$\begin{array}{lll}\text { L } & 1 & \text { L8 }\end{array}$

2 L9

3 -L8-L9

Dependent Variable: DADOS 
Source $\quad$ DF $\begin{gathered}\text { Sum of } \\ \text { Squares }\end{gathered}$ Square F Value $\operatorname{Pr}>F$

$\begin{array}{llllll}\text { Model } & 6 & 8.86271782 & 1.47711964 & 84.69 & 0.0001\end{array}$

$\begin{array}{llll}\text { Error } & 20 & 0.34884514 & 0.01744226\end{array}$

Corrected Total $\quad 26 \quad 9.21156296$

$\begin{array}{rrrc}\text { R-Square } & \text { C.V. } & \text { Root MSE } & \text { DADOS Mean } \\ 0.962130 & 11.51394 & 0.13207 & 1.14704\end{array}$

Source DF Type I SS Mean Square F Value $\operatorname{Pr}>\mathrm{F}$

$\begin{array}{lrrrrc}\mathrm{C} & 2 & 3.39069705 & 1.69534853 & 97.20 & 0.0001 \\ \mathrm{~T} & 2 & 0.08350307 & 0.04175153 & 2.39 & 0.1169 \\ \mathrm{~L} & 2 & 5.38851770 & 2.69425885 & 154.47 & 0.0001\end{array}$

Source DF Type II SS Mean Square F Value $\operatorname{Pr}>$ F
C
$\mathrm{T}$
$\begin{array}{lllll}2 & 1.67134249 & 0.83567125 & 47.91 & 0.0001\end{array}$
$\begin{array}{lllrr}2 & 0.06067515 & 0.03033758 & 1.74 & 0.2012\end{array}$
L
$\begin{array}{lllll}2 & 5.38851770 & 2.69425885 & 154.47 & 0.0001\end{array}$

Source DF Type III SS Mean Square F Value $\operatorname{Pr}>$ F
C
T
$\begin{array}{lllll}2 & 1.67134249 & 0.83567125 & 47.91 & 0.0001\end{array}$
$\begin{array}{lllll}2 & 0.06067515 & 0.03033758 & 1.74 & 0.2012\end{array}$
$\begin{array}{llllll}2 & 5.38851770 & 2.69425885 & 154.47 & 0.0001\end{array}$

Source

DF Type IV SS Mean Square F Value $\operatorname{Pr}>\mathrm{F}$

$\begin{array}{lrrrrr}\mathrm{C} & 2 & 1.67134249 & 0.83567125 & 47.91 & 0.0001 \\ \mathrm{~T} & 2 & 0.06067515 & 0.03033758 & 1.74 & 0.2012 \\ \mathrm{~L} & 2 & 5.38851770 & 2.69425885 & 154.47 & 0.0001\end{array}$

T for H0: $\quad \operatorname{Pr}>|\mathrm{T}| \quad$ Std Error of 


\begin{tabular}{|c|c|c|c|c|c|}
\hline \multirow{2}{*}{\multicolumn{2}{|c|}{$\begin{array}{l}\text { Parameter } \\
\text { INTERCEPT }\end{array}$}} & \multicolumn{2}{|c|}{ Estimate $\quad$ Parameter $=0$} & \multicolumn{2}{|c|}{ Estimate } \\
\hline & & & \multirow{2}{*}{$\begin{array}{c}20.37 \\
9.65\end{array}$} & \multirow{2}{*}{$\begin{array}{c}0.0001 \\
0.0001\end{array}$} & \multirow{2}{*}{$\begin{array}{r}0.07411793 \\
0.07060313\end{array}$} \\
\hline \multirow[t]{3}{*}{$\mathrm{C}$} & 1 & \multirow{2}{*}{$\begin{array}{c}\text { Estimate Paran } \\
\\
1.509559352 \mathrm{~B} \\
0.681625372 \mathrm{~B} \\
0269984354 \mathrm{~B}\end{array}$} & & & \\
\hline & 2 & & 3.91 & 0.0009 & 0.06905915 \\
\hline & 3 & $0.000000000 \mathrm{~B}$ & & & \\
\hline \multirow[t]{3}{*}{$\mathrm{T}$} & \multirow{3}{*}{$\begin{array}{l}2 \\
3\end{array}$} & $0.133123012 \mathrm{~B}$ & 1.85 & 0.0787 & 0.07183746 \\
\hline & & $0.018842977 \mathrm{~B}$ & 0.31 & 0.7620 & 0.06138356 \\
\hline & & $0.000000000 \mathrm{~B}$ & & & \\
\hline \multirow[t]{3}{*}{$\mathrm{L}$} & \multirow[b]{3}{*}{3} & $-1.077045501 \mathrm{~B}$ & -14.75 & 0.0001 & 0.07299579 \\
\hline & & $-1.042648251 \mathrm{~B}$ & -15.85 & 0.0001 & 0.06579476 \\
\hline & & $0.000000000 \mathrm{~B}$ & & & \\
\hline
\end{tabular}

NOTE: The $X^{\prime} X$ matrix has been found to be singular and a generalized inverse was used to solve the normal equations. Estimates followed by the letter ' $\mathrm{B}$ ' are biased, and are not unique estimators of the parameters. 
141.

The SAS System

OBS $\quad \mathrm{L} \quad \mathrm{C} \quad \mathrm{T}$ REP DADOS

$\begin{array}{cccccc}1 & 1 & 1 & 1 & 1 & 1.64 \\ 2 & 1 & 2 & 2 & 1 & 0.62 \\ 3 & 1 & 2 & 2 & 2 & 0.61 \\ 4 & 1 & 3 & 3 & 1 & 0.41 \\ 5 & 1 & 3 & 3 & 2 & 0.39 \\ 6 & 1 & 3 & 3 & 3 & 0.37 \\ 7 & 1 & 3 & 3 & 4 & 0.38 \\ 8 & 2 & 1 & 3 & 1 & 1.11 \\ 9 & 2 & 1 & 3 & 2 & 1.09 \\ 10 & 2 & 1 & 3 & 3 & 1.10 \\ 11 & 2 & 1 & 3 & 4 & 1.12 \\ 12 & 2 & 1 & 3 & 5 & 1.11 \\ 13 & 2 & 2 & 1 & 1 & 0.81 \\ 14 & 2 & 2 & 1 & 2 & 0.80 \\ 15 & 2 & 2 & 1 & 3 & 0.82 \\ 16 & 2 & 3 & 2 & 1 & 0.62 \\ 17 & 2 & 3 & 2 & 2 & 0.62 \\ 18 & 2 & 3 & 2 & 3 & 0.61 \\ 19 & 3 & 1 & 2 & 1 & 2.13 \\ 20 & 3 & 1 & 2 & 2 & 2.11 \\ 21 & 3 & 2 & 3 & 1 & 1.86 \\ 22 & 3 & 2 & 3 & 2 & 1.88 \\ 23 & 3 & 2 & 3 & 3 & 1.85 \\ 24 & 3 & 2 & 3 & 4 & 1.84 \\ 25 & 3 & 2 & 3 & 5 & 1.84 \\ 26 & 3 & 2 & 3 & 6 & 1.80 \\ 27 & 3 & 3 & 1 & 1 & 1.43\end{array}$


General Linear Models Procedure

Class Level Information

Class Levels Values

L $\quad 3 \quad 123$

C $\quad 3 \quad 123$

$\begin{array}{lll}\mathrm{T} & 3 & 123\end{array}$

Number of observations in data set $=27$

General Form of Estimable Functions

Effect Coefficients

INTERCEPT L1

T $1 \quad$ L2

$2 \quad \mathrm{~L} 3$

3 L1-L2-L3

L $\quad 1 \quad$ L5

$2 \quad \mathrm{~L} 6$

3 L1-L5-L6

$\begin{array}{lll}\text { C } & 1 & \text { L8 }\end{array}$

2. L9

3 L1-L8-L9 
Type I Estimable Functions for: T

Effect Coefficients

INTERCEPT 0

$\begin{array}{lll}\mathrm{T} & 1 & \mathrm{~L} 2\end{array}$

$2 \mathrm{~L} 3$

3 -L2-L3

L $\quad 1 \quad-0.0667 * \mathrm{~L} 2+0.019 * \mathrm{~L} 3$

$2 \quad 0.2667 * \mathrm{~L} 2+0.0952 * \mathrm{~L} 3$

$3 \quad-0.2 * \mathrm{~L} 2-0.1143 * \mathrm{~L} 3$

C $\quad 1 \quad-0.1333 *$ L2-0.0476*L3

$2 \quad 0.2 * \mathrm{~L} 2-0.1143 * \mathrm{~L} 3$

$3 \quad-0.0667 * \mathrm{~L} 2+0.1619 * \mathrm{~L} 3$

Type I Estimable Functions for: L

Effect Coefficients

INTERCEPT 0

$\begin{array}{lll}\mathrm{T} & 1 & 0 \\ & 2 & 0 \\ & 3 & 0\end{array}$

L $1 \quad$ L5

$2 \quad$ L6

3 -L5-L6

C $\quad 1 \quad-0.0681 * \mathrm{~L} 5+0.2699 * \mathrm{~L} 6$

$2 \quad-0.386 * \mathrm{~L} 5-0.4377 * \mathrm{~L} 6$

$3 \quad 0.4542 * \mathrm{~L} 5+0.1678 * \mathrm{~L} 6$ 
Type I Estimable Functions for: C

Effect Coefficients

INTERCEPT 0

$\begin{array}{lll}\text { T } & 1 & 0 \\ & 2 & 0 \\ & 3 & 0\end{array}$

$\begin{array}{lll}\mathrm{L} & 1 & 0 \\ & 2 & 0 \\ & 3 & 0\end{array}$

$\begin{array}{lll}\text { C } & 1 & \text { L8 }\end{array}$

$2 \quad$ L9

3 -L8-L9

Type II Estimable Functions for: $\mathbf{T}$

Effect Coefficients

INTERCEPT 0

$\begin{array}{lll}\text { T } & 1 & \text { L2 } \\ & 2 & \text { L3 } \\ & 3 & -\mathrm{L} 2-\mathrm{L} 3\end{array}$

L 110

20

30

$\begin{array}{lll}\text { C } & 1 & 0 \\ & 2 & 0\end{array}$

30 
Type II Estimable Functions for: L

Effect Coefficients

INTERCEPT 0

T $\quad 1 \quad 0$

30

L 11 L5

2 L6

3 -L5-L6

$\begin{array}{lll}\mathrm{C} & 1 & 0 \\ & 2 & 0\end{array}$

30

Type II Estimable Functions for: C

Effect Coefficients

INTERCEPT 0

$\begin{array}{lll}\mathrm{T} & 1 & 0\end{array}$

20

30

L 110

20

30

C $\quad 1 \quad$ L8

$2 \quad \mathrm{~L} 9$

$3 \quad-L 8-L 9$ 
Type III Estimable Functions for: T

Effect Coefficients

INTERCEPT 0

$\begin{array}{ccc}\text { T } & 1 & \text { L2 } \\ & 2 & \text { L3 } \\ & 3 & \text {-L2 } \\ & & \\ \text { L } & 1 & 0 \\ & 2 & 0 \\ & 3 & 0 \\ & & \\ \text { C } & 1 & 0 \\ & 2 & 0 \\ & 3 & 0\end{array}$

Type III Estimable Functions for: L

Effect Coefficients

INTERCEPT 0

$\begin{array}{lll}\text { T } & 1 & 0 \\ & 2 & 0 \\ & 3 & 0\end{array}$

L 1 L5

$2 \quad$ L6

3 -L5-L6

$\begin{array}{lll}\text { C } & 1 & 0 \\ & 2 & 0\end{array}$

30 
Type III Estimable Functions for: C

Effect Coefficients

INTERCEPT 0

$\begin{array}{lll}\text { T } & 1 & 0 \\ & 2 & 0 \\ & 3 & 0\end{array}$

L $\quad 1 \quad 0$

$\begin{array}{ll}2 & 0 \\ 3 & 0\end{array}$

C 11 L8

3 -L8-L9

Type IV Estimable Functions for: $T$

Effect Coefficients

INTERCEPT 0

$\begin{array}{lll}\text { T } & 1 & \text { L2 } \\ & 2 & \text { L3 } \\ & 3 & \text {-L2-L3 }\end{array}$

$\begin{array}{lll}\mathrm{L} & 1 & 0 \\ & 2 & 0\end{array}$

30

$\begin{array}{lll}\text { C } & 1 & 0 \\ & 2 & 0\end{array}$

$\begin{array}{ll}2 & 0 \\ 3 & 0\end{array}$ 
148.

Type IV Estimable Functions for: L

Effect Coefficients

\section{INTERCEPT 0}

$\begin{array}{lll}\text { T } & 1 & 0 \\ & 2 & 0 \\ & 3 & 0 \\ & & \\ \text { L } & 1 & \text { L5 } \\ & 2 & \text { L6 } \\ & 3 & \text {-L5-L6 } \\ & & \\ \text { C } & 1 & 0 \\ & 2 & 0 \\ & 3 & 0\end{array}$

Type IV Estimable Functions for: C

Effect Coefficients

INTERCEPT 0

$\mathrm{T}$

$\begin{array}{ll}1 & 0 \\ 2 & 0 \\ 3 & 0\end{array}$

$\begin{array}{lll}\mathrm{L} & 1 & 0 \\ & 2 & 0 \\ & 3 & 0\end{array}$

$\begin{array}{lll}\text { C } & 1 & \text { L8 }\end{array}$

$2 \quad$ L9

3 -L8-L9 


\begin{tabular}{|c|c|c|c|c|c|}
\hline \multirow[b]{2}{*}{ Source } & \multicolumn{2}{|r|}{ Sum of } & \multicolumn{3}{|l|}{ Mean } \\
\hline & DF & Squares & \multicolumn{2}{|c|}{ Square F Value } & $r>F$ \\
\hline Model & 6 & 8.86271782 & 1.47711964 & \multirow[t]{2}{*}{84.69} & 0.0001 \\
\hline Error & 20 & 0.34884514 & 0.01744226 & & \\
\hline \multicolumn{2}{|l|}{ Corrected Total } & 9.21156 & 296 & & \\
\hline $\mathrm{R}-\mathrm{Sq}$ & dare & C.V. & Root MSE & \multicolumn{2}{|c|}{ DADOS Mean } \\
\hline 0.96 & 130 & 11.51394 & 0.13207 & 1.1 & 704 \\
\hline
\end{tabular}

Source DF Type I SS Mean Square F Value $\operatorname{Pr}>$ F

$\begin{array}{llllrl}\mathrm{T} & 2 & 0.14239153 & 0.07119577 & 4.08 & 0.0326 \\ \mathrm{~L} & 2 & 7.04898379 & 3.52449190 & 202.07 & 0.0001 \\ \mathrm{C} & 2 & 1.67134249 & 0.83567125 & 47.91 & 0.0001\end{array}$

Source DF Type II SS Mean Square F Value $\operatorname{Pr}>$ F

$\begin{array}{llllrl}\mathrm{T} & 2 & 0.06067515 & 0.03033758 & 1.74 & 0.2012 \\ \mathrm{~L} & 2 & 5.38851770 & 2.69425885 & 154.47 & 0.0001 \\ \mathrm{C} & 2 & 1.67134249 & 0.83567125 & 47.91 & 0.0001\end{array}$

Source DF Type III SS Mean Square F Value $\operatorname{Pr}>$ F

$\begin{array}{llllrl}\mathrm{T} & 2 & 0.06067515 & 0.03033758 & 1.74 & 0.2012 \\ \mathrm{~L} & 2 & 5.38851770 & 2.69425885 & 154.47 & 0.0001 \\ \mathrm{C} & 2 & 1.67134249 & 0.83567125 & 47.91 & 0.0001\end{array}$

Source DF Type IV SS Mean Square F Value $\operatorname{Pr}>$ F
$\mathrm{T}$
$\begin{array}{lllll}2 & 0.06067515 & 0.03033758 & 1.74 & 0.2012\end{array}$
$\begin{array}{llllll}2 & 5.38851770 & 2.69425885 & 154.47 & 0.0001\end{array}$
L
$\begin{array}{lllll}2 & 1.67134249 & 0.83567125 & 47.91 & 0.0001\end{array}$ 
T for H0: $\quad \operatorname{Pr}>|\mathrm{T}| \quad$ Std Error of

\begin{tabular}{|c|c|c|c|c|c|}
\hline \multirow{2}{*}{\multicolumn{2}{|c|}{$\begin{array}{l}\text { Parameter } \\
\text { INTERCEPT }\end{array}$}} & \multicolumn{2}{|c|}{ Estimate $\quad$ Parameter $=0$} & \multicolumn{2}{|c|}{ Estimate } \\
\hline & & & & \multirow{2}{*}{$\begin{array}{c}0.0001 \\
0.0787\end{array}$} & \multirow{2}{*}{$\begin{array}{c}0.07411793 \\
0.07183746\end{array}$} \\
\hline \multirow[t]{3}{*}{$\mathbf{T}$} & 1 & \multirow{2}{*}{$\begin{array}{c}\text { Estimate Paran } \\
1.509559352 \mathrm{~B} \\
0.133123012 \mathrm{~B} \\
0.018842977 \mathrm{~B}\end{array}$} & & & \\
\hline & 2 & & \multirow{2}{*}{$\begin{array}{l}20.37 \\
1.85 \\
0.31\end{array}$} & \multirow{2}{*}{0.7620} & \multirow[t]{2}{*}{0.06138356} \\
\hline & 3 & $0.000000000 \mathrm{~B}$ & & & \\
\hline \multirow[t]{3}{*}{$\mathrm{L}$} & \multirow[t]{3}{*}{ 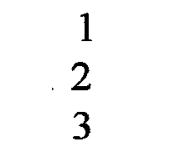 } & $-1.077045501 \mathrm{~B}$ & -14.75 & 0.0001 & 0.07299579 \\
\hline & & $-1.042648251 \mathrm{~B}$ & -15.85 & 0.0001 & 0.06579476 \\
\hline & & $0.000000000 \mathrm{~B}$ & & \multirow{4}{*}{$\begin{array}{l}0.0001 \\
0.0009\end{array}$} & \multirow{4}{*}{$\begin{array}{c}0.07060313 \\
0.06905915\end{array}$} \\
\hline \multirow[t]{3}{*}{$\mathrm{C}$} & \multirow[b]{3}{*}{3} & $0.681625372 \mathrm{~B}$ & 9.65 & & \\
\hline & & $0.269984354 \mathrm{~B}$ & 3.91 & & \\
\hline & & $0.000000000 \mathrm{~B}$ & & & \\
\hline
\end{tabular}

NOTE: The $\mathrm{X}^{\prime} \mathrm{X}$ matrix has been found to be singular and a generalized inverse was used to solve the normal equations. Estimates followed by the letter ' $\mathrm{B}$ ' are biased, and are not unique estimators of the parameters. 